\title{
Líquenes de Andalucía (S de España): catálogo bibliográfico y nuevos datos del NW del área
}

\author{
Ana Rosa Burgaz ${ }^{1}$
}

Resumen: Burgaz, A. R. 2014. Líquenes de Andalucía (S de España): catálogo bibliográfico y nuevos datos del NW del área. Bot. Complut. 38: 53-88.

Se aporta un catálogo de 1228 taxones (1185 ascolíquenes, 3 basidiolíquenes y 40 hongos liquenícolas) encontrados en Andalucía. El catá$\log _{0}$ está basado en referencias bibliográficas y en la revisión de especímenes depositados en el herbario MACB. De ellos, 11 son novedad para Andalucía, 122 lo son para la provincia de Córdoba, 1 para Granada, 3 para Huelva, 2 para Málaga y 1 para Sevilla. Se han excluido 74 especies, por no haber sido confirmada su presencia con posterioridad a su única cita.

Palabras clave: hongos liquenizados, hongos liquenícolas, España meridional.

Abstract: Burgaz, A. R. 2014. Lichens of Andalusia (S of Spain): bibliographic checklist and new data from the north western area. Bot. Complut. 38: 53-88

A catalogue of 1228 taxa (1185 ascolichens, 3 basidiolichens and 40 lichenicolous fungi) found in Andalusia is afforded. The catalogue is based on bibliographic references and on MACB herbarium specimens. Of them, 11 are new records in Andalusia, 122 in Cordoba province, 1 in Granade province, 3 in Huelva province, 2 in Malaga province and 1 in Seville province. We propose the exclusion of 74 old references, because of the lack of any confirmation

Key words: lichen-forming fungi, lichenicolous fungi, southern Spain.

\section{INTRODUCCIÓN}

La Comunidad Autónoma de Andalucía, situada al sur de la Península Ibérica, con una extensión de $87.268 \mathrm{~km}^{2}$, está dividida en 7 provincias y constituye el mayor territorio de la Península (Fig. 1). Está atravesada por el valle del río Guadalquivir, delimitado al norte por el sistema montañoso de Sierra Morena y al SE por las Sierras Béticas, donde se encuentran las mayores alturas peninsulares. Esto implica la existencia de un medio físico muy variado, desde el nivel del mar hasta los $3.482 \mathrm{~m}$ de altitud del pico Mulhacén, lo que permite la existencia de numerosos biotopos, también debidos, entre otras causas, a la existencia de substratos geológicos muy diversos. El territorio es muy contrastado en las precipitaciones, pues en él encontramos áreas con precipitación muy escasa $(\mathrm{Pm}=231 \mathrm{~mm}$ en Almería, $18 \mathrm{~m})$ y otras con el máximo de precipitación peninsular (Grazalema, Cádiz, $\mathrm{Pm}=2.223 \mathrm{~mm}, 823 \mathrm{~m})$. En general, las temperaturas me- dias anuales son suaves, desde $10,1^{\circ} \mathrm{C}$ en Arroyo Canales (Jaén, $1240 \mathrm{~m}$ ) hasta $19,2^{\circ} \mathrm{C}$ en Lora del Río (Sevilla, $38 \mathrm{~m}$ ), aunque en las zonas culminales de las sierras, las temperaturas medias son inferiores (Rivas-Martínez 1987).

Pertenece a la región Mediterránea, en la que se distinguen varias provincias y subprovincias corológicas (Peinado Lorca \& Rivas-Martínez 1987, Rivas Martínez et al. 2002). La subprovincia Luso-Extremadurense ocupa la zona noroccidental del territorio ( $S^{\mathrm{a}}$ de Aroche, valle de los Pedroches, parte occidental de $S^{a}$ Morena y estribaciones); se caracteriza por penillanuras paleozoicas con pequeños desniveles. En su mayor parte se incluye en el piso mesomediterráneo con ombroclima subhúmedo, y está dominada por encinares adehesados, alcornocales y charnecales, con pequeños enclaves de acebuchales. La subprovincia Gaditano-Algarviense, con las sierras del Aljibe y de Algeciras, ocupa una pequeña área en el SO del territorio, con altitudes inferiores a los $700 \mathrm{~m}$, presenta un bioclima termomediterráneo, de ombroclima subhúmedo

\footnotetext{
${ }^{1}$ Departamento de Biología Vegetal I, Facultad Ciencias Biológicas, Universidad Complutense de Madrid, E-28040 Madrid, España. arburgaz@bio.ucm.es

Recibido: 30 abril 2014. Aceptado: 28 mayo 2014.
} 

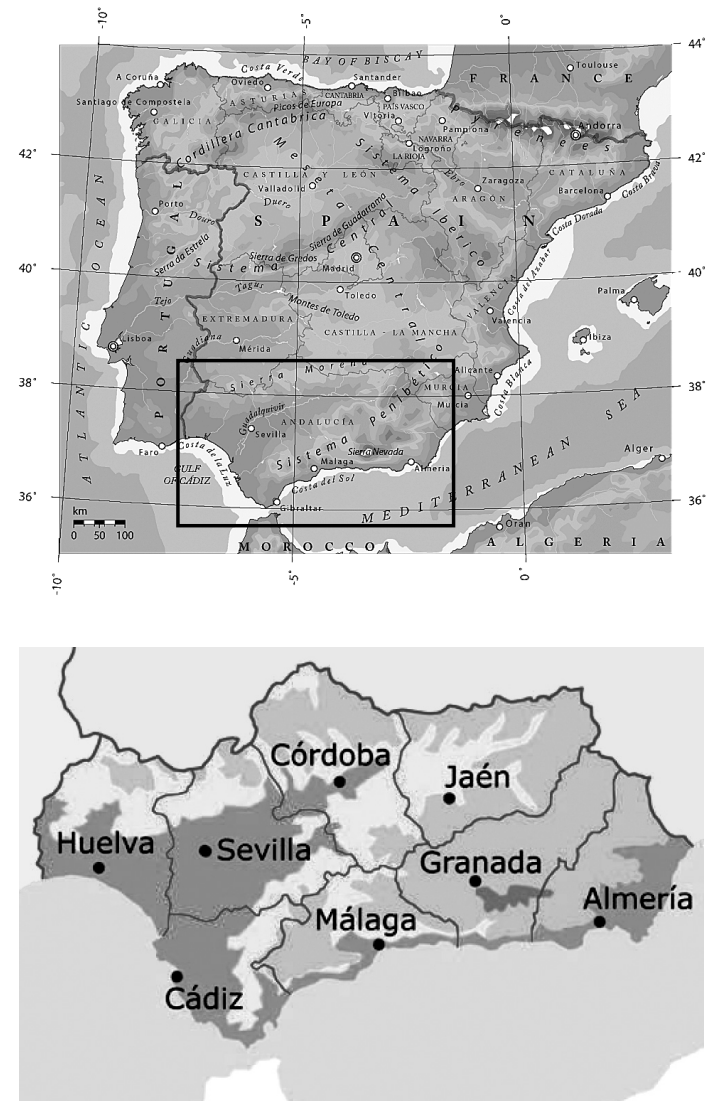

Fig. 1- Localización de Andalucía en la Península Ibérica.

hasta hiperhúmedo, con ausencia de heladas, donde dominan las dunas, marismas, estuarios, areniscas y arcillas; la vegetación varía desde las formaciones psammófilas y de helófitos, en dunas y marismas, hasta los acebuchales, alcornocales, quejigares de Quercus canariensis, y comunidades riparias, como fresnedas, saucedas y alisedas. La provincia Bética es la más amplia y compleja, ya que comprende la depresión del Guadalquivir y las Sierras Béticas. Posee gran diversidad de substratos geológicos, ácidos y básicos. En ella aparecen todos los pisos bioclimáticos existentes en la Península Ibérica y numerosos ombroclimas. Además, se encuentran las mayores altitudes de la Península, con el pico Mulhacén de 3.482 $\mathrm{m}$, Veleta de $3.395 \mathrm{~m}$ y La Alcazaba de $3.371 \mathrm{~m}$. Esta provincia se subdivide en el sector Hispalense que ocupa toda la depresión del río Guadalquivir, está constituido por materiales sedimentarios ricos en bases, principalmente dedicados al cultivo. El sector Rondense con la Serranía de Ronda y aledaños, alcanza elevaciones en torno a los 1.900 m; está constituido por substratos ácidos y básicos de distinta naturaleza, sobre los que aparecen alcornocales, encinares, abetales y quejigares de Quercus faginea. El sector Malacitano-Almijarense con las sierras de la Almijara y de los Filabres cuyas cotas están próximas a los $2.000 \mathrm{~m}$, con bioclimas meso- y supramediterráneos, presenta encinares, matorrales abiertos y tomillares en las zonas más bajas sobre substratos calizos; encinares y enebrales rastreros en los afloramientos silíceos de la $\mathrm{S}^{\mathrm{a}}$ de los Filabres. El sector Subbético, con las sierras de Mágina con $2.164 \mathrm{~m}$, Cazorla y Segura, con cotas algo inferiores está en general, dominado por las calizas, en muy diversas formas; se encuentran en él encinares basófilos, que ocupan los pisos meso- y supramediterráneo, acompañados por quejigos en algunos valles y umbrías; el piso oromediterráneo está ocupado por pinares de Pinus sylvestris y P. nigra. El sector Nevadense comprende la Sierra Nevada, con varias cumbres que superan los 3.000 m; aparecen en él substratos silíceos y calizos, que están colonizados por coscojares, encinares, melojares, enebrales rastreros y pastizales psicroxerófilos, entre otras formaciones vegetales. El sector Alpujarreño-Gadorense ocupa las vertientes meridionales de la $S^{a}$ Nevada, e incluye las sierras de la Contraviesa y de Gádor, con 2.236 $\mathrm{m}$; los substratos son silíceos, calizos, o de margas y arcillas carbonatadas; se desarrollan en él sabinares rastreros, encinares y algunos alcornocales, en los pisos oro-, supray mesomediterráneo. El sector Guadijeño-Baztetano, con las sierras de Guadix con $2.270 \mathrm{~m}$, y de Baza, está ocupado por substratos silíceos, calizos, yesosos y margosos, que son colonizados por encinares, quejigares y pinares. La provincia Murciano-Almeriense ocupa el SE del territorio, posee un ombroclima semiárido y constituye uno de los territorios más secos de toda la Península Ibérica. Aparecen en ella lentiscares, espinales y cornicales termomediterráneos, coscojares termo- y mesomediterráneos; el substrato geológico es muy variado, ya que aparecen materiales carbonatados, afloramientos yesosos, materiales silíceos y volcánicos, estos últimos en la $S^{\mathrm{a}} \mathrm{del}$ Cabo de Gata y aledaños. En el NE del territorio, aparece un pequeño enclave, perteneciente al sector manchego de la subprovincia Castellana, que está ocupado con coscojares y encinares mesomediterráneos basófilos.

\section{RESULTADOS}

El estudio de los líquenes de Andalucía comienza a la vez que se inicia el desarrollo de la liquenología en España, con la obra de Clemente $(1807,1863)$ (Llimona \& Hladun 2001) que, junto con Asso (1779), puede considerar- 
se uno de los padres de la liquenología española. Clemente publica una lista de 122 taxones, la mayoría procedentes de la provincia de Cádiz y otros datos inéditos, con varias especies nuevas, que fueron recopilados por Colmeiro (1868). Una parte del herbario Boutelou, actualmente en la Universidad de Sevilla, posee 11 pliegos recogidos por Clemente en Andalucía, pero no se encuentra entre ellos ninguna de las especies nuevas descritas por él (Silvestre \& García Rowe 1982). Paralelamente, Lallave (1820), sacerdote de origen mexicano, publica algunos líquenes nuevos de América y recolecta material de la provincia de Cádiz. Este autor, al igual que Clemente y Lagasca, fue responsable de la introducción en España de la obra de Acharius (González-Bueno 1988, González-Bueno \& Rico 1991). Desgraciadamente, todo este trabajo no ha podido ser valorado, ya que el material de herbario sobre el que se sustenta no ha podido áun ser encontrado. Willkomm, durante los años 1844, 1845 y 1850, realiza expediciones por España, con el objetivo de estudiar la flora vascular y recolecta varios líquenes por Andalucía, que fueron, probablemente, revisados por Flotow (Sampaio 1917). Este material permanece en el herbario del Jardín Botánico de Oporto (Portugal). Posteriormente, se realizan algunas aportaciones en el territorio por naturalistas españoles como González-Fragoso (1883) y Barras (1896, 1899), cuyo material fue revisado por Nylander (Silvestre \& García-Rowe 1982), Lázaro-Ibiza (1898a, b) y Navás (1902). También la visita de criptogamistas extranjeros a España aporta novedades para Andalucía. Entre los más destacados son las aportaciones de Werner $(1975,1979)$, que posteriormente colaborará con Xavier Llimona.

Hasta la década de los 70 no aparecen nuevas publicaciones de líquenes con referencias andaluzas, procedentes de la provincia corológica Murciano-Almeriense (Llimona 1974). Gracias al nuevo destino del Profesor Llimona en la Universidad de Murcia (1977-1982), se inicia una época floreciente en el estudio de las biotas liquénicas del S y SE peninsular, con las investigaciones llevadas a cabo por José $\mathrm{M}^{\mathrm{a}}$ Egea, Pilar Torrente, Pedro Pablo Moreno y Leandro Alonso (en la provincia Murciano-Almeriense y el sector Malacitano-Almijarense), Manuel Casares (en el sector Nevadense y Rondense), Jorge García Rowe y Xavier Ariño (en la subprovincia Gaditano-Algarviense), entre otros. Por último hay que destacar a Victor Jiménez Rico y Gregorio Aragón, por las aportaciones realizadas en el sector Subbético.

Debido a la ausencia de datos en la subprovincia Luso-Extremadurense, se ha recolectado nuevo material, que se encuentra depositado en el herbario MACB y que procede en su mayor parte de la provincia de Córdoba. Se han muestreado localidades del Valle de los Pedroches situado al norte de la provincia y donde dominan las dehesas de encinas y substratos graníticos; hacia el centro se ha prospectado Sierra Morena y enclaves pertenecientes a los Parques Naturales de Cardeña-Montoro y sierra de Hornachuelos situados al noreste y al oeste de la provincia respectivamente, con altitudes que oscilan entre 100-800 m y substratos ácidos. Por último, al sur de la provincia y en las zonas bajas del Parque Natural de las Sierras Subbéticas, hasta $970 \mathrm{~m}$ de altitud, y donde dominan los substratos calcáreos. En la provincia de Sevilla se han recogido ejemplares en la zona oriental del Parque Natural de la Sierra Norte, con encinas y alcornoques que crecen sobre substratos ácidos.

Se han aceptado los últimos cambios nomenclaturales en Teloschistaceae que afectan a los géneros Caloplaca, Fulgensia y Xanthoria (Arup et al. 2013); Aspicilia (Sohrabi et al. 2013); Arthoniales (Ertz et al. 2013); Diploschistes (Kraichak et al. 2014); Melanelia (Divakar et al. 2012) y Verrucaria (Gueidan et al. 2007).

De entre las especies identificadas, 11 son novedad para Andalucía, y se indican en el listado en negrita y con el símbolo $(* *)$. Las novedades provinciales se señalan en negrita y con el símbolo (*), y corresponden 122 a la provincia de Córdoba, 1 a la de Granada, 3 a la de Huelva, 2 a la Málaga y 1 a la de Sevilla. El catálogo totaliza 1228 taxones, 1185 ascolíquenes, 3 basidiolíquenes y 40 hongos liquenícolas, señalados en el texto con el símbolo (\#). Se han excluido 74 especies, por no haber sido confirmada su presencia con posterioridad a la cita. Las abreviaturas provinciales son las habituales para la Flora Ibérica.

\#Abrothallus microspermus Tul. - Ca: Rowe et al. (1996).

Acarospora badiofusca (Nyl.) Th. Fr. - Al: Egea \& Llimona (1981a, 1983, 1987); Egea et al. (1982).

Acarospora bulgarica H. Magn. \& Szatala - Al: Egea \& Llimona (1981a, 1983).

Acarospora bullata Anzi -Al: Egea \& Llimona (1981a).

Acarospora cervina (Ach.) A. Massal. - Ca: Casares \& Rowe (1988), Rowe et al. (1996); Gr: Casares \& Llimona (1986, 1989), Casares (1988); Ma: Herrera et al. (2007).

Acarospora charidema (Colmeiro) Llimona -Al: Colmeiro (1867), Llimona (1975), Egea \& Llimona (1981a, b, 1983, 1985, 1987, 1994).

Acarospora clauzadeana (Llimona) Casares \& Hafellner-Al: Llimona (1974), Casares \& Gutiérrez (1993), Gutiérrez \& Casares (1994), Guerra et al. (1995).

Acarospora epithallina H. Magn. - Al: Egea \& Llimona (1981a, b, 1983, 1987); Ca: Rowe \& Egea (1988); *Co: MACB 103150; J: Crespo et al. (1976); Se: Rowe \& Egea (1985a, b).

Acarospora fuscata (Nyl.) Arnold - Al: Llimona (1975), Egea \& Llimona (1981a, 1983, 1985, 1994); Ca: Rowe \& Egea (1986, 1988), Egea \& Rowe (1987); Gr: Egea et al. (1982), Egea \& Llimona (1983); H: Rowe \& Egea (1987a); Se: Rowe \& Egea (1985a, b, 1987a). 
Acarospora glaucocarpa (Ach.) Körb. - Ca: Casares \& Rowe (1988); J: Sampaio (1917).

Acarospora heufleriana Körb. - Al: Llimona (1975), Egea \& Llimona (1981b, 1983, 1987, 1994, 1997).

Acarospora hilaris (Dufour) Arnold -Al: Egea \& Llimona (1981a, b, 1983, 1987); Ca: Rowe \& Egea (1986, 1988); *Co: MACB 103151; H: Rowe \& Egea (1987a); J: Crespo et al. (1976); Ma: Seaward \& Arvidson (1997); Se: Llimona \& Egea (1985), Rowe \& Egea $(1985 \mathrm{a}, \mathrm{b})$

Acarospora hospitans H. Magn. -Al: Egea \& Llimona (1981a, 1983, 1987, 1994); Gr: Egea et al. (1982); Se: Rowe \& Egea (1987a).

Acarospora impressula Th. Fr. -Al: Egea \& Llimona (1981a, 1983, 1987).

Acarospora laqueata Stizenb. - Gr: Casares \& Llimona (1983, 1986, 1989), Casares (1986).

Acarospora macrospora (Hepp) A. Massal. ex Bagl. -Al: Egea \& Llimona (1994); Gr: Casares \& Llimona (1983, 1986, 1989); J: Aragón et al. (2006).

Acarospora macrospora subsp. murorum (A. Massal.) Clauzade \& $\mathrm{Cl}$. Roux - Al: Gutiérrez \& Casares (1994); Ca: Rowe et al. (1996); Gr: Casares (1988), Casares \& Llimona (1989).

Acarospora maroccana B. de Lesd. - Al: Egea \& Llimona (1981a, b, 1983, 1985, 1987, 1994).

Acarospora microcarpa (Nyl.) Wedd. - Al: Egea \& Llimona (1983, 1994).

Acarospora modenensis H. Magn - Gr: Pereira et al. (1987).

Acarospora nodulosa var. reagens (Zahlbr.) Clauzade \& Cl. Roux Al: Llimona (1974), Casares \& Gutiérrez (1993), Gutiérrez \& Casares (1994), Alonso \& Egea (1996); Gr: Casares \& Llimona (1989), Casares (1992), Casares \& Gutiérrez (1993).

Acarospora oligospora (Nyl.) Arnold - Ca: Rowe \& Egea (1988).

Acarospora picea H. Magn. - Gr: Egea et al. (1982).

Acarospora placodiiformis H. Magn. - Al: Llimona (1974), Casares \& Gutiérrez (1993), Gutiérrez \& Casares (1994); Gr: Casares \& Gutiérrez (1993).

Acarospora scotica Hue - Ca: Rowe \& Egea (1986, 1988); H: Rowe \& Egea (1987a); Se: Rowe \& Egea (1985b).

Acarospora smaragdula (Wahlenb.) A. Massal. - Ca: Rowe \& Egea (1988); Gr: Egea (1985); H: Rowe et al. (1982); Se: Rowe \& Egea (1985b, 1987a).

Acarospora sphaerospora H. Magn. - Al: Egea \& Llimona (1981a, 1983).

Acarospora subrufula (Nyl.) H. Olivier-Al: Egea \& Llimona (1981b, 1983, 1987)

Acarospora sulphurata (Arnold) Arnold-Al: Egea \& Llimona (1981a, 1983, 1987); Gr: Egea \& Llimona (1983).

Acarospora umbilicata Bagl. - Al: Egea \& Llimona (1981b, 1983, 1987, 1994); Ca: Rowe \& Egea (1988); *Co: MACB 106704; H: Rowe \& Egea (1987a); Se: Rowe \& Egea (1985b, 1987a).

Acarospora veronensis A. Massal. - Se: Rowe \& Egea (1987a).

Acarospora versicolor Bagl. \& Carestia - H: Rowe et al. (1982).

Agonimia allobata (Stizenb.) P. James - Ca: Rowe et al. (1996); J: Aragón \& Sarrión (2003).

Agonimia octospora Coppins \& P. James - Ca: Rowe et al. (1996); J: Aragón \& Sarrión (2003); Ma: Rowe et al. (1996).

Agonimia opuntiella (Buschardt \& Poelt) Vêzda - J: Aragón \& Sarrión (2003).

Agonimia tristicula (Nyl.) Zahlbr. - J: Aragón \& Sarrión (2003); Ma: Rowe et al. (1996).

Ainoa mooreana (Carroll) Lumbsch \& I. Schmitt - Ca: Rowe \& Egea (1988); Se: Rowe \& Egea (1987a).
Alyxoria ochrocincta (Werner) Ertz - Al: Torrente \& Egea (1989a). Alyxoria varia (Pers.) Ertz \& Tehler - Al: Torrente \& Egea (1989a); Ca: Colmeiro (1867), Barreno et al. (1988), Rowe et al. (1996). Amandinea maritima Giralt et al. - Ma: Lumbsch et al. (2011).

Amandinea punctata (Hoffm.) Coppins \& Scheid. - Al: Egea \& Llimona (1983, 1987), Casares \& Gutiérrez (1993); Ca: Colmeiro (1867), Barreno et al. (1988), Fos (1998); H: Fos (1998); J: Aragón et al. (2006a); Ma: Martín-Osorio (1988), Rowe et al. (1996).

Anaptychia ciliaris (L.) Körb. - Al: Colmeiro (1867), Egea \& Llimona (1981a, 1987); Ca: Colmeiro (1867), Barreno et al. (1988), Rowe \& Egea (1988), Rowe et al. (1996); Fos (1998); *Co: MACB 103727; Gr: Colmeiro (1867), Casares \& Llimona (1986, 1989), Fos (1998); J: Aragón et al. (2006a), Aragón \& Rico (1997); Ma: Abbayes (1945), Martín-Osorio (1988), Rowe et al. (1996); Fos (1998).

Anaptychia runcinata (With.) J. R. Laundon - Ca: Colmeiro (1867), Rowe \& Egea (1986).

Anema nodulosum (Nyl.) Forssell - Gr: Casares \& Llimona (1989), Moreno \& Egea (1992); Ma: Herrera et al. (2007).

Anema nummularium (Dufour) Nyl. - Al: Moreno \& Egea (1992); Ca: Rowe et al. (1996); Gr: Casares \& Llimona (1989), Moreno \& Egea (1992); Ma: Moreno \& Egea (1992).

Anema prodigulum (Nyl.) Henssen -Al: Moreno \& Egea (1992); Ma: Herrera et al. (2007).

Anisomeridium polypori (Ellis \& Everh.) M. E. Barr - Ca: Rowe et al. (1996).

Anthracocarpon virescens (Zahlbr.) Breuss - Al: Gutiérrez \& Casares (1994), Alonso \& Egea (1996), Prieto et al. (2010); Ca: Prieto et al. (2010); *Co: MACB 103725; Gr: Prieto et al. (2010); J: Prieto et al. (2010); Ma: Prieto et al. (2010).

Arthonia albopulverea Nyl. -Al: Grube \& Giralt (1996); Ma: Herrera et al. (2007).

Arthonia atra (Pers.) A. Schneid. - Ca: Colmeiro (1867), Barreno et al. (1988), Fos (1998); Gr: Colmeiro (1867); H: Torrente \& Egea (1989a).

Arthonia calcarea (Turner ex Sm.) Ertz \& Diederich - Ca: Ariño et al. (1997); Gr: Casares \& Llimona (1989).

Arthonia calcicola Nyl. - Ma: Herrera et al. (2007).

Arthonia cinnabarina (DC.) Wallr. - Ca: Colmeiro (1867), Fos (1998).

Arthonia melanophthalma Dufour - Ma: Herrera et al. (2007).

Arthonia meridionalis Zahlbr. - Al: Alonso \& Egea (1996).

Arthonia pinastri Anzi - J: Aragón et al. (2006a).

Arthonia pruinata (Pers.) Steud. ex A. L. Sm. - Ca: Rowe et al. (1996).

Arthonia punctiformis Ach. - Ca: Fos (1998); J: Aragón et al. (2006a).

Arthonia radiata (Pers.) Ach. - Al: Colmeiro (1867); Ca: Colmeiro (1867); Gr: Colmeiro (1867); Ma: Herrera et al. (2007).

Arthopyrenia cinereopruinosa (Schaer.) A. Massal. - Ca: Rowe et al. (1996).

Arthopyrenia punctiformis (Schrank) A. Massal. - Ca: Barreno et al. (1988); Ma: Herrera et al. (2007).

Arthothelium sardoum Bagl. - Al: Grube \& Giralt (1996); Ca: Fos (1998).

Aspicilia caecula (Ach.) Anzi -Al: Egea \& Llimona (1981a, 1987).

Aspicilia calcarea var. reagens (Zahlbr.) Szatala - Al: Egea \& Llimona (1981a, 1987).

Aspicilia cheresina (Müll. Arg.) Hue -Al: Alonso \& Egea (1996); Gr: Casares \& Llimona (1989).

Aspicilia cheresina var. justii (Servít) Clauzade \& Cl. Roux - Ca: Casares \& Rowe (1988); Gr: Casares \& Llimona (1986, 1989).

Aspicilia cheresina var. microspora (Arnold) Clauzade \& Cl. Roux Ca: Casares \& Rowe (1988). 
Aspicilia cinerea (L.) Körb. -Al: Egea \& Llimona (1981a, 1987); Ca: Colmeiro (1867); Ma: Colmeiro (1867); Se: Barras (1896).

Aspicilia contorta subsp. hoffmanniana S. Ekman \& Fröberg ex R. Sant. - Al: Egea \& Llimona (1981a, b, 1985, 1987, 1994), Casares \& Gutiérrez (1993), Gutiérrez \& Casares (1994); Ca: Rowe \& Egea (1986, 1988), Egea \& Rowe (1987), Ariño et al. (1995), Rowe et al. (1996); Gr: Casares \& Llimona (1982), Casares \& Gutiérrez (1993); H: Rowe et al. (1982), Rowe \& Egea (1987a); Se: Llimona \& Egea (1985), Rowe \& Egea (1985a, b, 1987a), Rowe \& Sainz-Jiménez (1988), Puertas et al. (1994), Ariño et al. (1997).

Aspicilia coronata (A. Massal.) B. de Lesd. - Ca: Casares \& Rowe (1988); Gr: Casares \& Llimona (1986, 1989).

Aspicilia cupreoglauca B. de Lesd. - Al: Egea \& Llimona (1981a, 1987); Gr: Egea et al. (1982).

Aspicilia cupreogrisea (Th. Fr.) Hue - Al: Egea \& Llimona (1981a); Gr: Egea et al. (1982), H: Rowe et al. (1982).

Aspicilia epiglypta (Norrl. ex Nyl.) Hue -Al: Egea \& Llimona (1981a, 1987); Gr: Egea et al. (1982).

Aspicilia excipularis (H. Magn.) Creveld -Al: Egea \& Llimona (1981a, 1987); Gr: Egea et al. (1982).

Aspicilia helvola Werner - Al: Egea \& Llimona (1981a, 1987).

Aspicilia intermutans (Nyl.) Arnold -Al: Llimona (1975), Egea \& Llimona (1981a, b, 1985, 1987, 1994); Ca: Rowe \& Egea (1986, 1988), Egea \& Rowe (1987), Rowe et al. (1996); *Co: MACB 106705; Gr: Casares \& Llimona (1982); H: Rowe et al. (1982), Rowe \& Egea (1987a); Se: Rowe \& Egea (1985a, b; 1987a).

Aspicilia laevata (Ach.) Arnold - Gr: Pereira et al. (1987).

Aspicilia lignicola (Anzi) Hue - J: Aragón et al. (2004c).

Aspicilia mastrucata (Wahlenb.) Th. Fr. - Gr: Egea (1985).

Aspicilia polychroma Anzi - Al: Egea \& Llimona (1987).

Aspicilia polychroma f. verruculosa Egea - Al: Egea \& Llimona (1981a); Gr: Egea et al. (1982), Egea (1985).

Aspicilia recedens (Taylor) Arnold - Al: Egea \& Llimona (1981a).

Aspicilia supertegens Arnold - Gr: Egea (1985).

Aspicilia uxoris (Werner) V. J. Rico et al. - J: Rico et al. (2007).

Athallia alnetorum (Giralt et al.) Arup et al. - Ma: Rowe et al. (1996), Herrera et al. (2007).

Athallia cerinella (Nyl.) Arup et al. - Gr: Fos (1998).

Athallia cerinelloides (Erichsen) Arup et al. - Ca: Fos (1998); Gr: Fos (1998).

Athallia holocarpa (Hoffm.) Arup et al. -Al: Egea \& Llimona (1994); Ca: Barreno et al. (1988), Rowe et al. (1996), Fos (1998); *Co: MACB 103324; Gr: Egea (1984), Casares \& Llimona (1989), Fos (1998); H: Rowe et al. (1982), Fos (1998); J: Aragón et al. (2004c, 2006a); Ma: Rowe et al. (1996), Fos (1998), Herrera et al. (2007).

Bacidia absistens (Nyl.) Arnold - Ca: Rowe et al. (1996), Fos (1998), Llop \& Hladun (2002), Llop (2007).

Bacidia arceutina (Ach.) Rehm \& Arnold - Ca: Barreno et al. (1988), Rowe et al. (1996), Llop \& Hladun (2002), Llop (2007); J: Llop (2007).

Bacidia auerswaldii (Hepp ex Stizenb.) Mig. - Ca: Llop (2007); Se: Llop \& Hladun (2002).

Bacidia bagliettoana (A. Massal. \& De Not.) Jatta - Gr: Casares (1988), Casares \& Llimona (1989), Llop \& Hladun (2002); J: Aragón et al. (2006a); Ma: Martínez \& Hafellner (1998).

Bacidia circumspecta (Norrl. \& Nyl.) Malme - Ca: Rowe et al. (1996), Llop \& Hladun (2002), Llop (2007); J: Aragón \& Sarrión (2003), Llop (2007); Ma: Rowe et al. (1996).

Bacidia fraxinea Lönnr. - Ca: Rowe et al. (1996), Llop \& Gómez-Bolea (1999), Llop \& Hladun (2002).
Bacidia friesiana (Hepp) Körb. - Ca: Llop \& Hladun (2002), Llop (2007).

Bacidia iberica Aragón \& Martínez - J: Llop (2007).

Bacidia igniarii (Nyl.) Oxner -Al: Llop \& Hladun (2002), Llop (2007); Gr: Llop (2007); J: Aragón \& Sarrión (2003).

Bacidia laurocerasi (Delise ex Duby) Zahlbr. - Ca: Barreno et al. (1988), Rowe et al. (1996), Llop \& Hladun (2002), Llop (2007).

Bacidia parathalassica Llop \& Gómez-Bolea - Al: Llop \& GómezBolea (1999), Llop (2007); Ca: Llop \& Gómez-Bolea (1999), Llop \& Hladun (2002), Llop (2007).

Bacidia phacodes Körb. - Ca: Rowe et al. (1996).

Bacidia propinqua (Hepp ex Stizenb.) Arnold - Ca: Fos (1998).

Bacidia rosella (Pers.) De Not. - Ca: Rowe et al. (1996), Llop \& Hladun (2002), Llop (2007); J: Llop (2007); Ma: Seaward \& Arvidson (1997), Llop \& Hladun (2002).

Bacidia rubella (Hoffm.) A. Massal. - Ca: Rowe et al. (1996), Fos (1998), Llop \& Gómez-Bolea (1999), Llop \& Hladun (2002), Llop (2007); J: Aragón \& Sarrión (2003), Aragón \& Martínez (2003), Llop (2007); Ma: Rowe et al. (1996), Seaward \& Arvidson (1997); Llop \& Gómez-Bolea (1999), Llop \& Hladun (2002), Llop (2007).

Bacidia subacerina Vain. - Ca: Rowe et al. (1996).

Bacidina assulata (Körb.) S. Ekman - Ma: Llop (2007).

Bacidina delicata (Larbal. ex Leight.) V. Wirth \& Vězda - Ca: Llop (2007).

Bacidina egenula (Nyl.) Vězda - Ca: Rowe \& Egea (1986), Egea \& Rowe (1987), Llop (2007).

Bacidina phacodes (Körb.) Vězda - Al: Ca: Llop (2007); Ca: Llop (2007); Ma: Llop (2007).

Bactrospora patellarioides (Nyl.) Almq. -Al: Torrente \& Egea (1989a); Ca: Rowe \& Egea (1986), Barreno et al. (1988), Torrente \& Egea (1989a), Egea \& Torrente (1993), Fos (1998); Ma: Egea \& Torrente (1993).

Bagliettoa baldensis (DC) Gueidan \& Cl. Roux - Al: Alonso \& Egea (1996); Gr: Alonso \& Egea (1996).

Bagliettoa calciseda (DC) Gueidan \& Cl. Roux - Al: Alonso \& Egea (1996); Ca: Casares \& Rowe (1988), Alonso \& Egea (1996), Rowe et al. (1996); Gr: Casares \& Llimona (1984, 1986), Alonso \& Egea (1996); Ma: Colmeiro (1867), Herrera et al. (2007).

Bagliettoa marmorea (DC) Gueidan \& Cl. Roux - Ca: Rowe et al. (1996); *Co: MACB 103894; Gr: Casares \& Llimona $(1984,1986)$.

Bagliettoa parmigera (J. Steiner) Gueidan \& Cl. Roux - Gr: Casares \& Llimona $(1984,1986)$.

Biatorella clauzadeana Llimona - Al: Llimona (1974).

\#Biatoropsis usnearum Räsänen - Ca: Rowe et al. (1996); Ma: Rowe et al. (1996).

Bilimbia sabuletorum (Schreb.) Arnold - Ca: Rowe et al. (1996); J: Aragón et al. (2006a); Ma: Rowe et al. (1996).

Biscogniauxia repanda (Fr.) Kuntze - Al: Egea \& Llimona (1983, 1984).

Blastenia crenularia (With.) Arup et al. -Al: Egea \& Llimona (1981a, b, 1983, 1997), Egea (1984); Ca: Egea (1984), Rowe \& Egea (1986, 1988), Egea \& Rowe (1987), Egea \& Llimona (1994), Rowe et al. (1996); Co: Egea (1984); Gr: Casares \& Llimona (1982); H: Rowe et al. (1982), Egea (1984); Ma: Egea (1984); Se: Egea (1984), Llimona \& Egea (1985), Rowe \& Egea (1985b, 1987a).

Blastenia ferruginea (Huds.) A. Massal. - Al: Colmeiro (1867); Ca: Colmeiro (1867), Barreno et al. (1988), Rowe et al. (1996), Fos (1998); Gr: Colmeiro (1867), Fos (1998); H: Fos (1998); J: Aragón et al. (2004c); Ma: Colmeiro (1867), Martín-Osorio (1988), Rowe et al. (1996), Fos (1998). 
Blastenia herbidella (Hue) Servít - J: Aragón et al. (2004c, 2006a); Ma: Martín-Osorio (1988), Rowe et al. (1996), van den Boom \& Rico (2006).

Blastenia hungarica (H. Magn.) Arup et al. - Ca: Barreno et al. (1988), Fos (1998); Gr: Fos (1998); H: Fos (1998); Ma: Martín-Osorio (1988).

Botryolepraria lesdainii (Hue) Canals et al. - Ca: Rowe et al. (1996), Ariño et al. (1997); Se: Ariño et al. (1997).

Brodoa intestiniformis (Vill.) Goward - Gr: Colmeiro (1867), Egea et al. (1982)

Bryoria capillaris (Ach.) Brodo \& D. Hawksw. - J: Aragón et al. (2006a).

Bryoria chalybeiformis (L.) Brodo \& D. Hawksw. - Ma: Colmeiro (1867).

Bryoria fuscescens (Gyeln.) Brodo \& D. Hawksw. - Gr: Colmeiro (1867); J: Aragón et al. (2006a), Aragón \& Rico (1997); Ma: Colmeiro (1867).

Bryoria implexa (Hoffm.) Brodo \& D. Hawksw. - J: Aragón et al. (2006a).

Buellia aethalea (Ach.) Th. Fr. - Ma: Herrera et al. (2007).

Buellia almeriensis Llimona - Al: Llimona (1974), Casares (1992), Casares \& Gutiérrez (1993), Gutiérrez \& Casares (1994), Guerra et al. (1995).

Buellia badia (Fr.) A. Massal. - Ca: Egea \& Rowe (1987), Rowe \& Egea (1988); H: Rowe \& Egea (1987a), Fos (1998); Se: Rowe \& Egea (1985a, 1987a)

Buellia cedricola Werner - J: Sarrión \& Burgaz (1995), Aragón et al. (2004c).

Buellia disciformis (Fr.) Mudd - Ca: Barreno et al. (1988), Rowe et al. (1996), Fos (1998); H: Fos (1998); J: Aragón et al. (2006a).

Buellia dispersa A. Massal. - Al: Egea \& Llimona (1981a, b, 1983, 1985, 1987, 1994, 1997); Ca: Rowe \& Egea (1986), Egea \& Rowe (1987); Ma: Herrera et al. (2007); Se: Rowe \& Egea (1985b).

Buellia enteroleucoides (Nyl.) Arnold - Gr: Egea et al. (1982).

Buellia epigaea (Hoffm.) Tuck. -Al: Casares \& Gutiérrez (1993), Gutiérrez \& Casares (1994).

Buellia erubescens Arnold - Ca: Barreno et al. (1988); H: Fos (1998).

Buellia glaucoatra (Nyl.) Clauzade - Al: Egea \& Llimona (1981a, 1983, 1987); Ca: Rowe \& Egea (1986); Gr: Egea et al. (1982).

Buellia griseovirens (Turner \& Borrer ex Sm.) Almb. - H: Fos (1998); J: Aragón et al. (2004c, 2006a); Ma: Martín-Osorio (1988), Rowe et al. (1996), Herrera et al. (2007).

Buellia heliophila Llimona - Al: Llimona (1974), Gutiérrez \& Casares (1994), Guerra et al. (1995).

Buellia iberica Giralt - J: Aragón et al. (2006a).

Buellia italica A. Massal. - Al: Egea \& Llimona (1994).

Buellia lactea (A. Massal.) Körb. - Ca: Rowe \& Egea (1986), Egea \& Rowe (1987); Se: Rowe \& Egea (1985b, 1987a).

Buellia lainea (Ach.) Clauzade - Al: Egea \& Llimona (1987).

Buellia lauri-cassiae (Fée) Müll. Arg. - Ma: Rowe et al. (1996).

Buellia leptocline A. Massal. - Al: Egea \& Llimona (1981a, 1983, 1987); Ca: Rowe \& Egea (1986); Gr: Egea et al. (1982); Se: Rowe \& Egea (1985b, 1987a).

Buellia leptoclinoides (Nyl.) J. Steiner - Al: Llimona (1975), Egea \& Llimona (1994); Ca: Rowe \& Egea (1986), Egea \& Rowe (1987), Rowe et al. (1996).

Buellia lusitanica J. Steiner - Ca: Rowe \& Egea (1986).

Buellia nivalis (Bagl. \& Carestia) Hertel - Al: Egea (1985b), Casares \& Gutiérrez (1993), Gutiérrez \& Casares (1994); Gr: Casares \& Llimona (1989), Egea (1985b).
Buellia saxorum A. Massal. - Ca: Rowe \& Egea (1986), Egea \& Rowe (1987).

Buellia schaereri De Not. - Ca: Fos (1998).

Buellia sequax (Nyl.) Zahlbr. -Al: Egea \& Llimona (1994).

Buellia spuria (Schaer.) Anzi - Gr: Casares \& Llimona (1982).

Buellia squamulata (Nyl.) Zahlbr. - Se: Rowe \& Egea (1985b).

Buellia stellulata (Taylor) Mudd - Ca: Rowe \& Egea (1986, 1988); Ma: Herrera et al. (2007).

Buellia subdisciformis (Leight.) Jatta - Al: Egea \& Llimona (1981a, b, 1983, 1987); Ca: Rowe \& Egea (1986, 1988), Egea \& Rowe (1987); Ma: Herrera et al. (2007).

Buellia subsquamosa J. Steiner - Al: Egea \& Llimona (1981a, 1987); H: Rowe et al. (1982), Rowe \& Egea (1987a); Se: Rowe \& Egea (1987a).

Buellia tesserata Körb. - Al: Llimona (1975), Llimona \& Werner (1975), Egea \& Llimona (1983, 1984, 1994), Rico et al. (2003); Ca: Rowe \& Egea (1986), Egea \& Rowe (1987); Ma: Herrera et al. (2007).

Buellia triphragmia (Nyl.) Arnold - Ca: Fos (1998); H: Fos (1998).

Buellia triseptata A. Nordin - Ca: Giralt \& Nordin (2002); H: Giralt \& Nordin (2002); J: Aragón et al. (2006a).

Buellia tumida (A. Massal.) Bagl. -Al: Egea \& Llimona (1981b, 1983, 1987).

Buellia zoharyi Galun - Al: Llimona (1974), Casares \& Gutiérrez (1993), Gutiérrez \& Casares (1994), Trinkaus \& Mayrhofer (2000); Gr: Casares \& Llimona $(1983,1989)$, Casares \& Gutiérrez (1993), Martínez-Sánchez et al. (1994).

Calicium abietinum Pers. J: Sarrión et al. (1999), Aragón et al. (2004c, 2006a), Muñiz \& Hladun (2011).

Calicium adspersum Pers. - Ca: Colmeiro (1867); H: Colmeiro (1867); Ma: Rowe et al. (1996), Sarrión et al. (1999), Muñiz \& Hladun (2011).

Calicium chlorosporum F. Wilson - Ma: Sarrión et al. (2007), Muñiz \& Hladun (2011).

Calicium corynellum (Ach.) Ach. - Se: Sarrión et al. (1999).

Calicium glaucellum Ach. - J: Sarrión et al. (1999), Aragón et al. (2004c, 2006a), Muñiz \& Hladun (2011); Ma: Seaward \& Arvidson (1997), Muñiz \& Hladun (2011).

Calicium montanum Tibell - J: Aragón et al. (2004c), Muñiz \& Hladun (2011).

Calicium parvum Tibell - J: Sarrión \& Burgaz (1995).

Calicium salicinum Pers. - Ca: Colmeiro (1867); J: Sarrión et al. (1999), Aragón et al. (2006a), Muñiz \& Hladun (2011); Ma: Rowe et al. (1996), Sarrión et al. (1999), Muñiz \& Hladun (2011).

Calicium viride Pers. - H: Fos (1998); J: Sarrión et al. (1999), Aragón et al. (2006a), Muñiz \& Hladun (2011).

Calogaya biatorina (A. Massal.) Arup et al. - Gr: Egea (1984), Casares \& Llimona (1986, 1989), Casares (1988).

Calogaya decipiens (Arnold) Arup et al. - *Co: MACB 103321; Gr: Egea (1984), Casares \& Llimona (1986, 1989).

Calogaya pusilla (A. Massal.) Arup et al. - Al: Colmeiro (1867), Alonso \& Egea (1996); Ca: Colmeiro (1867), Sampaio (1917); Gr: Colmeiro (1867), Egea (1984); Ma: Colmeiro (1867); Se: Barras (1896).

Calogaya schistidii (Anzi) Arup et al. - Ca: Casares \& Rowe (1988), Rowe et al. (1996); Gr: Poelt (1965), Casares (1988), Casares \& Llimona (1989); Ma: Rowe et al. (1996), Seaward \& Arvidson (1997).

Caloplaca aegatica Giralt et al. - Ca: Fos (1998).

Caloplaca aetnensis B. de Lesd. - Al: Egea \& Llimona (1983, 1987), Egea (1984), Llimona \& Egea (1985); Ma: Seaward \& Arvidson (1997), Herrera et al. (2007). 
Caloplaca agardhiana (Flot.) Flagey -Al: Alonso \& Egea (1996); Ca: Casares \& Rowe (1988); Gr: Casares \& Llimona $(1986,1989)$.

Caloplaca ameliensis (Nyl.) H. Olivier-Al: Egea \& Llimona (1981a, b, 1983, 1987), Egea (1984).

Caloplaca anchon-phoeniceon Poelt \& Clauzade - Gr: Casares \& Llimona (1982), Egea (1984).

Caloplaca aractina (Fr.) Häyrén - Al: Egea \& Llimona (1981a).

Caloplaca asserigena (J. Lahm) Della Torre \& Sarnth. - Ca: Fos (1998).

Caloplaca biatorina var. baumgartneri (Zahlbr.) Poelt - Al: Egea (1984), Gr: Egea (1984).

Caloplaca cerina var. cerina (Ehrh. ex Hedw.) Th. Fr. - Ca: Colmeiro (1867), Rowe et al. (1996), Fos (1998); Gr: Colmeiro (1867), Casares \& Llimona (1982), Fos (1998); J: Aragón et al. (2004c); Ma: Colmeiro (1867), Rowe et al. (1996), Fos (1998), Martínez \& Hafellner (1998), Martínez (1999), Herrera et al. (2007).

Caloplaca cerina var. chloroleuca (Sm.) Th. Fr. - Al: Egea \& Llimona (1981a); Gr: Colmeiro (1867), Casares \& Llimona (1982, 1989), Egea et al. (1982), Casares (1986), Egea \& Llimona (1987), Casares (1988); J: Aragón et al. (2006a).

Caloplaca chlorina (Flot.) Sandst. - Al: Egea (1984); Gr: Pereira et al. (1987).

Caloplaca circumalbata (Delise) Wunder - Al: Tretiach \& Muggia (2006); Gr: Casares \& Llimona (1986).

Caloplaca circumalbata var. candida (Stizenb.) Wunder - Al: Egea (1984); Gr: Casares \& Llimona (1989).

Caloplaca concinerascens (Nyl.) H. Olivier - Al: Alonso \& Egea (1996).

Caloplaca conglomerata (Bagl.) Jatta. - Al: Egea \& Llimona (1983), Llimona \& Egea (1985), Egea \& Llimona (1987); J: Egea (1984); H: Rowe \& Egea (1987a); Se: Egea (1984), Rowe \& Egea (1985a, b, 1987a).

Caloplaca conglomerata var. nigrescens Egea \& Llimona - Al: Egea \& Llimona (1981a).

Caloplaca conversa (Kremp.) Jatta - Al: Egea (1984), Egea \& Llimona (1994), Alonso \& Egea (1996); Gr: Alonso \& Egea (1996).

Caloplaca conversa var. cravensis Clauzade \& Wunder - Gr: Egea (1984), Casares \& Llimona (1989).

Caloplaca coronata (Kremp.) Jatta - Ca: Casares \& Rowe (1988); Gr: Egea (1984), Casares (1988), Casares \& Llimona (1986, 1989); Ma: Herrera et al. (2007).

Caloplaca crenularia (With.) J. R. Laundon - Al: Egea \& Llimona (1983), Egea (1984), Egea \& Llimona (1987); Ca: Rowe \& Egea (1988).

Caloplaca dalmatica (A. Massal.) H. Olivier -Al: Egea \& Llimona (1984), Alonso \& Egea (1996); Ca: Casares \& Rowe (1988), Ariño et al. (1995, 1997), Alonso \& Egea (1996); Gr: Egea (1984), Casares (1988), Casares \& Llimona (1989); Ma: Seaward \& Arvidson (1997), Herrera et al. (2007); Se: Ariño et al. (1997).

Caloplaca epithallina Lynge - Al: Egea \& Llimona (1981a, 1983, 1987); Gr: Casares \& Llimona (1982), Egea et al. (1982), Egea (1984).

Caloplaca exsecuta (Nyl.) Dalla Torre \& Sarnth. - Gr: Egea et al. (1982), Egea (1984)

Caloplaca furax Egea \& Llimona - H: Rowe \& Egea (1987a), Se: Rowe \& Egea (1987a).

Caloplaca fuscolivida (Müll. Arg.) Zahlbr. - Ca: Rowe et al. (1996).

Caloplaca gloriae Llimona \& Werner - Al: Llimona (1975), Llimona \& Werner (1975), Egea (1984), Egea \& Llimona (1994).
Caloplaca haematites (Chaub. ex St.-Amans) Zwackh - Ca: Fos (1998); Ma: Martín-Osorio (1988), Rowe et al. (1996).

Caloplaca inconnexa (Nyl.) Zahlbr. - Al: Egea \& Llimona (1981a, b, 1994), Casares \& Gutiérrez (1993), Gutiérrez \& Casares (1994); Ca: Casares \& Rowe (1988), Rowe et al. (1996); Gr: Egea (1984), Casares \& Llimona $(1986,1989)$.

Caloplaca insularis Poelt - Gr: Egea (1984).

Caloplaca irrubescens (Arnold) Zahlbr. - Al: Llimona (1975), Egea \& Llimona (1981a, b, 1983, 1987, 1994, 1997), Egea (1984), Llimona \& Egea (1985); Ca: Egea (1984), Rowe \& Egea (1986); Gr: Egea (1984); H: Rowe et al. (1982); J: Egea (1984); Ma: Herrera et al. (2007); Se: Egea (1984), Rowe \& Egea (1985a, b, 1987a).

Caloplaca lecideina (Müll. Arg.) Clauzade \& Rondon - Al: Egea \& Llimona (1981a, 1987).

Caloplaca ligustica B. de Lesd. - Al: Egea \& Llimona (1981b, 1983, 1987), Egea (1984).

Caloplaca littorea Tav. - Ma: Herrera et al. (2007).

Caloplaca lucifuga G. Thor - Ma: Rowe et al. (1996).

Caloplaca marina Wedd. - Ma: Seaward \& Arvidson (1997), Herrera et al. (2007).

Caloplaca necator Poelt \& Clauzade - Al: Llimona (1975), Egea (1984), Egea \& Llimona $(1987,1994)$.

Caloplaca nubigena (Kremp.) Dalla Torre \& Sarnth. - Gr: Egea (1984).

Caloplaca obscurella (J. Lahm) Th. Fr. - Ca: Fos (1998); Gr: Fos (1998); H: Fos (1998); Ma: Rowe et al. (1996), Fos (1998).

Caloplaca paulii Poelt - Al: Egea \& Llimona (1981a), Egea (1984).

Caloplaca pelodella (Nyl.) Hasse - J: Paz-Bermúdez et al. (2006).

Caloplaca phlogina (Ach.) Flagey - Ma: Martín-Osorio (1988).

Caloplaca pollinii (A. Massal.) Jatta - Ca: Barreno et al. (1988), Fos (1998); H: Fos (1998).

Caloplaca quercina Flagey - Ca: Barreno et al. (1988).

Caloplaca rubelliana (Ach.) Lojka-Al: Egea \& Llimona (1981a, 1983, 1987, 1994), Egea (1984).

Caloplaca sarcopidoides (Körb.) Zahlbr. - Ca: Fos (1998); H: Fos (1998).

'Caloplaca' teicholyta (Ach.) J. Steiner - Al: Egea \& Llimona (1994, 1997); Ca: Ariño et al. (1995), Alonso \& Egea (1996); Gr: Casares \& Llimona (1986, 1989); Se: Puertas et al. (1994), Ariño et al. (1997).

Caloplaca tenuatula (Nyl.) Zahlbr. - Al: Alonso \& Egea (1996); Ca: Alonso \& Egea (1996), Rowe et al. (1996); Gr: Egea (1984).

Caloplaca ulcerosa Coppins \& P. James - Ma: Rowe et al. (1996).

Caloplaca velana var. placidia (A. Massal.) Clauzade \& Cl. Roux Ca: Casares \& Rowe (1988).

Caloplaca velana var. velana (A. Massal.) Du Rietz - Gr: Casares \& Llimona (1986).

Caloplaca vitellinula (Nyl.) H. Olivier -Al: Egea (1984), Egea \& Llimona (1987).

Caloplaca xerica Poelt \& Vêzda - Al: Egea (1984), Egea \& Llimona (1994); Se: Rowe \& Egea (1987a).

Calvitimela aglaea (Sommerf.) Hafellner - Ca: Rowe \& Egea (1986, 1988), Egea \& Rowe (1987); Gr: Egea et al. (1982).

Candelaria concolor (Dicks.) Arnold - Ca: Fos (1998); Gr: Fos (1998); J: Aragón et al. (2004c, 2006a).

Candelariella aurella (Hoffm.) Zahlbr. - Al: Casares \& Gutiérrez (1993), Gutiérrez \& Casares (1994), Alonso \& Egea (1996); Ca: Casares \& Rowe (1988); Gr: Egea et al. (1982); Casares (1988), Casares \& Llimona (1986, 1989); Ma: Herrera et al. (2007); Se: Ariño et al. (1997).

Candelariella coralliza (Nyl.) H. Magn. - Ca: Egea \& Rowe (1987), Rowe \& Egea (1987b), Rowe et al. (1996). 
Candelariella medians (Nyl.) A. L. Sm. - Gr: Casares \& Llimona (1983, 1986, 1989).

Candelariella reflexa (Nyl.) Lettau - Ca: Rowe et al. (1996), Fos (1998); *Co: MACB 103897; H: Fos (1998).

Candelariella viae-lacteae $\mathrm{G}$. Thor \& V. Wirth - J: Aragón et al. (2004c).

Candelariella vitellina (Ehrht) Müll. Arg. - Al: Llimona (1975), Egea \& Llimona (1981a, 1987, 1994, 1997), Llimona \& Egea (1985); Ca: Colmeiro (1867), Rowe \& Egea $(1986,1988)$, Egea \& Rowe (1987), Fos (1998); *Co: MACB 103900; Gr: Degelius (1966), Casares \& Llimona (1982, 1986), Egea et al. (1982), Casares (1988), Fos (1998); H: Rowe \& Egea (1987a), Fos (1998); J: Aragón et al. (2004c, 2006a); Ma: Rowe et al. (1996), Herrera et al. (2007); Se: Rowe \& Egea (1985a, b, 1987a), Rowe \& Sainz-Jiménez (1988).

Candelariella vitellina var. assericola Räsänen - Gr: Casares \& Llimona (1989).

Candelariella xanthostigma (Pers. ex Ach.) Lettau - Ca: Fos (1998); J: Aragón et al. (2006a); Ma: Rowe et al. (1996).

Canoparmelia crozalsiana (B. de Lesd.) Elix \& Hale-Ca: Fos (1998).

\#Capronia peltigerae (Fuckel) D. Hawksw. - J: Martínez \& Hafellner (1998), Martínez (1999); Ma: Martínez \& Hafellner (1998), Martínez (1999).

\#Carbonea vitellinaria (Nyl.) Hertel - Gr: Egea (1985).

Carbonea vorticosa (Flörke) Hertel - Ca: Rowe \& Egea (1988).

Catapyrenium cinereum (Pers.) Körb. - Gr: Colmeiro (1867), Casares \& Llimona (1984); J: Prieto et al. (2010).

Catapyrenium daedaleum (Kremp.) Stein - Al: Prieto et al. (2010); Gr: Prieto et al. (2010); J: Aragón (2003); Ma: Prieto et al. (2010).

Catillaria chalybeia (Borrer) A. Massal. -Al: Egea \& Llimona (1981a, 1987, 1994), Alonso \& Egea (1996); Gr: Casares \& Llimona (1986, 1989); H: Fos (1998); J: Aragón et al. (2004c); Ma: Herrera et al. (2007)

Catillaria detractula (Nyl.) H. Olivier - Gr: Casares \& Llimona (1989).

Catillaria lenticularis (Ach.) Th. Fr. - Ca: Rowe et al. (1996); Gr: Casares \& Llimona (1989).

Catillaria mediterranea Hafellner - Ma: Rowe et al. (1996).

Catillaria nigroclavata (Nyl.) J. Steiner - Ca: Barreno et al. (1988), Rowe et al. (1996), Fos (1998); J: Aragón et al. (2004c, 2006a); Ma: Rowe et al. (1996).

Catillaria praedicta Tretiach \& Hafellner - Ca: Fos (1998), Tretiach \& Hafellner (1998).

Catinaria atropurpurea (Schaer.) Vězda \& Poelt - Ma: Rowe et al. (1996).

\#Cercidospora crozalsiana (H. Olivier) Nav.-Ros. et al. - Al: Navarro-Rosinés et al. (1995); Gr: Navarro-Rosinés et al. (1995); Se: Navarro-Rosinés et al. (1995).

\#Cercidospora epipolytropa (Mudd) Arnold - Gr: Degelius (1966).

Cetraria aculeata (Schreb.) Fr. - Ca: Colmeiro (1867), Rowe \& Egea (1987b), Rowe et al. (1996); H: Hernández Gallego \& Muñoz Reinoso (2005); J: Aragón \& Rico (1997); Se: Rowe \& Egea (1985b, 1987a).

Cetraria crespoae (Barreno \& Vázquez) Kärnefelt - H: Fos (1998).

Cetraria muricata (Ach.) Eckfeldt - Ca: Barreno et al. (1988).

Cetrariella commixta (Nyl.) A. Thell \& Kärnefelt - Ca: Colmeiro (1867); Gr: Casares \& Llimona (1982).

Cetrelia olivetorum (Nyl.) W. L. Culb. \& C. F. Culb. - Ca: Werner (1975), Barreno et al. (1988).

Chaenotheca brunneola (Ach.) Müll. Arg. - J: Sarrión et al. (1999),
Aragón et al. (2006a), Muñiz \& Hladun (2011); Ma: Sarrión et al. (1999), Muñiz \& Hladun (2011).

Chaenotheca chrysocephala (Turner ex Ach.) Th. Fr. - J: Sarrión et al. (1999), Aragón et al. (2006a), Muñiz \& Hladun (2011); Ma: Rowe et al. (1996).

Chaenotheca ferruginea (Turner ex Sm.) Mig. - J: Sarrión et al. (1999), Aragón et al. (2006a), Muñiz \& Hladun (2011).

Chaenotheca furfuracea (L.) Tibell - J: Sarrión et al. (1999), Aragón et al. (2006a), Muñiz \& Hladun (2011).

Chaenotheca hispidula (Ach.) Zahlbr. - Ca: Rowe et al. (1996); Ma: Rowe et al. (1996).

Chaenotheca phaeocephala (Turner) Th. Fr. - J: Sarrión et al. (1999), Aragón et al. (2006a), Muñiz \& Hladun (2011); Ma: Muñiz \& Hladun (2011).

Chaenotheca trichialis (Ach.) Th. Fr. - Gr: Colmeiro (1867); J: Sarrión et al. (1999), Aragón et al. (2006a), Muñiz \& Hladun (2011).

Chaenothecopsis debilis (Sm.) Tibell - Ma: Muñiz \& Hladun (2011).

Chaenothecopsis hospitans (Th. Fr.) Tibell - J: Muñiz \& Hladun (2011).

\#Chaenothecopsis pusilla (A. Massal.) Alb. Schmidt - Ma: Rowe et al. (1996), Sarrión et al. (1999), Muñiz \& Hladun (2011).

Chaenothecopsis subparoica (Nyl.) Tibell - Gr: Muñiz \& Hladun (2011).

Chaenothecopsis vainioana (Nádv.) Tibell - Ma: Muñiz \& Hladun (2011).

Chrysothrix candelaris (L.) J. R. Laundon - Ca: Barreno et al. (1988), Rowe et al. (1996), Fos (1998); H: Fos (1998); J: Aragón et al. (2006a).

Chrysothrix chlorina (Ach.) J. R. Laundon - Gr: Egea et al. (1982).

Circinaria caesocinerea (Nyl. ex Malbr.) A. Nordin et al. - Al: Egea \& Llimona (1981a, 1987); Ca: Fos (1998), Rowe \& Egea (1988); Gr: Colmeiro (1867), Egea et al. (1982), Pereira et al. (1987); H: Rowe et al. (1982); Ma: Herrera et al. (2007); Se: Rowe \& Egea (1985a).

Circinaria calcarea (L.) A. Nordin et al. - Al: Egea \& Llimona (1994), Alonso \& Egea (1996); Ca: Colmeiro (1867), Casares \& Rowe (1988); *Co: MACB 103726; Gr: Colmeiro (1867), Sampaio (1917), Casares (1988), Casares \& Llimona (1986, 1989), Alonso \& Egea (1996); J: Colmeiro (1867); Ma: Colmeiro (1867), Mottershead \& Lucas (2000), Herrera et al. (2007); Se: Barras (1899).

Circinaria contorta (Hoffm) A. Nordin et al. - Al: Alonso \& Egea (1996); Ca: Casares \& Rowe (1988), Rowe et al. (1996); Gr: Casares (1988), Casares \& Llimona (1986, 1989), Alonso \& Egea (1996); J: Aragón et al. (2006); Ma: Herrera et al. (2007).

Circinaria fruticulosa (Eversm.) Sohrabi - Ma: Follmann \& Crespo (1974).

Circinaria hispida (Merschk.) A. Nordin et al. - J: Aragón \& Rico (1997).

Cladonia caespiticia (Pers.) Flörke - Gr: Burgaz \& Ahti (2009).

Cladonia cariosa (Ach.) Spreng. - Gr: Burgaz \& Ahti (2009), PinoBodas et al. (2012a).

Cladonia cervicornis (Ach.) Flot. subsp. cervicornis - Ca: Burgaz \& Ahti (2009); H: Burgaz \& Ahti (2009), Pino-Bodas et al. (2010); J: Burgaz \& Ahti (2009); Ma: Burgaz \& Ahti (2009); Se: Burgaz $\&$ Ahti (2009).

Cladonia cervicornis subsp. verticillata (Hoffm.) Ahti - Ca: Abbayes (1945), H: Rowe \& Egea (1987a), Hernández Gallego \& Muñoz Reinoso (2005); Se: Rowe \& Egea (1987a).

Cladonia chlorophaea (Flörke ex Sommerf.) Spreng. -Al: Egea \& Lli- 
mona (1981a); Ca: Burgaz \& Ahti (2009); Gr: Burgaz \& Ahti (2009); H: Fos (1998), Hernández Gallego \& Muñoz Reinoso (2005), Burgaz \& Ahti (2009); J: Aragón et al. (2006a), Aragón \& Rico (1997); Ma: Burgaz \& Ahti (2009).

Cladonia ciliata Stirt. - Ca: Burgaz \& Ahti (2009); Ma: Burgaz \& Ahti (2009).

Cladonia coccifera (L.) Willd. - Ca: Colmeiro (1867), Barreno et al. (1988); H: Rowe et al. (1982); Rowe \& Egea (1987a).

Cladonia coniocraea (Flörke) Spreng. - Ca: Rowe et al. (1996); H: Rowe et al. (1982), Fos (1998); J: Aragón et al. (2006a), Burgaz \& Ahti (2009).

Cladonia corsicana (Rondon \& Vězda) Pino-Bodas et al. - Ca: PinoBodas et al. (2012b); Co: Pino-Bodas et al. (2012b); Se: PinoBodas et al. (2012b).

Cladonia cyathomorpha Stirt. ex Walt.Watson - Ca: Burgaz \& Ahti (2009); Co: Burgaz \& Ahti (2009); Gr: Burgaz \& Ahti (2009); H: Burgaz \& Ahti (2009); J: Aragón et al. (2006a); Ma: Burgaz \& Ahti (2009); Se: Burgaz \& Ahti (2009).

Cladonia dimorpha S. Hammer - Co: Burgaz \& Ahti (2009); H: Burgaz \& Ahti (2009); J: Burgaz \& Ahti (2009); Se: Burgaz \& Ahti (2009).

Cladonia diversa Asperges - J: Burgaz (2009), Burgaz \& Ahti (2009); Se: Burgaz (2009), Burgaz \& Ahti (2009).

Cladonia fimbriata (L.) Fr. - Al: Egea \& Llimona (1981a), Burgaz \& Ahti (2009); Ca: Rowe et al. (1996), Fos (1998), Burgaz \& Ahti (2009); Co: Burgaz \& Ahti (2009); Gr: Burgaz \& Ahti (2009); H: Rowe et al. (1982), Rowe \& Egea (1987a), Fos (1998); J: Aragón et al. (2006a), Aragón \& Rico (1997), Burgaz \& Ahti (2009); Ma: Rowe et al. (1996), Burgaz \& Ahti (2009); Se: Barras (1896), Burgaz \& Ahti (2009)

Cladonia firma (Nyl.) Nyl. - Ca: Burgaz \& Ahti (2009); H: Burgaz \& Ahti (2009); J: Burgaz \& Ahti (2009); Se: Rowe \& Egea (1987a), Burgaz \& Ahti (2009)

Cladonia foliacea (Huds.) Willd. -Al: Navás (1904), Abbayes (1945), Egea \& Llimona (1981b, 1994), Casares \& Gutiérrez (1993), Gutiérrez \& Casares (1994), Martínez-Sánchez et al. (1994), Alonso \& Egea (1996), Burgaz \& Ahti (2009); Ca: Colmeiro (1867), Navás (1904), Rowe \& Egea (1986, 1988), Burgaz \& Ahti (2009), Pino-Bodas et al. (2010); Co: Navás (1904); Burgaz \& Ahti (2009); Gr: Colmeiro (1867), Casares \& Llimona (1989), Casares \& Gutiérrez (1993), Alonso \& Egea (1996), Burgaz \& Ahti (2009), Pino-Bodas et al. (2010); H: Follmann (1973), Rowe et al. (1982), Rowe \& Egea (1987a), Hernández Gallego \& Muñoz Reinoso (2005), Burgaz \& Ahti (2009); J: Colmeiro (1867), Aragón \& Rico (1997), Burgaz \& Ahti (2009); Ma: Colmeiro (1867), Barras (1896), Sampaio (1917), Abbayes (1945), Burgaz \& Ahti (2009); Se: Barras (1896, 1899), Silvestre \& Rowe (1982), Burgaz \& Ahti (2009).

Cladonia furcata (Huds.) Schrad. -Al: Colmeiro (1867), Egea \& Llimona (1994), Burgaz \& Ahti (2009); Ca: Colmeiro (1867), Burgaz \& Ahti (2009); Gr: Colmeiro (1867), Navás (1904), Burgaz \& Ahti (2009); J: Burgaz \& Ahti (2009); Ma: Colmeiro (1867), Burgaz \& Ahti (2009); Se: Lázaro Ibiza (1898b).

Cladonia glauca Flörke - H: Burgaz \& Ahti (2009); J: Aragón et al. (2006a); Se: Burgaz \& Ahti (2009).

Cladonia gracilis (L.) Willd. - Ca: Rowe \& Egea (1988); Gr: Colmeiro (1867); Se: Lázaro Ibiza (1898b).

Cladonia hammeri Ahti - Ca: Burgaz \& Ahti (2009); Co: Burgaz \& Ahti (2009); Gr: Burgaz \& Ahti (2009); J: Burgaz \& Ahti (2009); Ma: Burgaz \& Ahti (2009); Se: Burgaz \& Ahti (2009).
Cladonia humilis (With.) J. R. Laundon - Ca: Burgaz \& Ahti (2009); Co: Burgaz \& Ahti (2009); Gr: Burgaz \& Ahti (2009); H: Burgaz \& Ahti (2009); J: Aragón et al. (2006a), Burgaz \& Ahti (2009); Ma: Burgaz \& Ahti (2009); Se: Burgaz \& Ahti (2009).

Cladonia luteoalba A. Wilson \& Wheldon - H: Burgaz (2009), Burgaz \& Ahti (2009).

Cladonia macilenta Hoffm. - Ca: Burgaz (2009), Burgaz \& Ahti (2009) J: Aragón \& Rico (1997).

Cladonia mediterranea P. A. Duvign. \& Abbayes - Ca: Burgaz \& Ahti (2009); H: Hernández Gallego \& Muñoz Reinoso (2005), Burgaz \& Ahti (2009).

Cladonia ochrochlora Flörke - J: Lázaro Ibiza (1898b), Aragón et al. (2006a), Burgaz \& Ahti (2009).

Cladonia phyllophora Ehrh. ex Hoffm. - Ca: Burgaz \& Ahti (2009); Co: Burgaz \& Ahti (2009); Gr: Colmeiro (1867), Burgaz \& Ahti (2009); H: Burgaz \& Ahti (2009); J: Burgaz \& Ahti (2009); Ma: Burgaz \& Ahti (2009); Se: Burgaz \& Ahti (2009).

Cladonia pocillum (Ach.) O. J. Rich. -Al: Abbayes (1945), Casares \& Gutiérrez (1993), Gutiérrez \& Casares (1994), Alonso \& Egea (1996), Burgaz \& Ahti (2009); Ca: Burgaz \& Ahti (2009); Co: Burgaz \& Ahti (2009); Gr: Casares (1988), Casares \& Llimona (1989), Casares \& Gutiérrez (1993), Martínez-Sánchez et al. (1994), Alonso \& Egea (1996), Burgaz \& Ahti (2009); J: Aragón \& Rico (1997), Burgaz \& Ahti (2009); Ma: Burgaz \& Ahti (2009); Se: Barras (1896).

Cladonia prolifica S. Hammer \& Ahti - Ca: Burgaz \& Ahti (2009); Co: Burgaz \& Ahti (2009); H: Burgaz \& Ahti (2009); Ma: Burgaz \& Ahti (2009); Se: Burgaz \& Ahti (2009).

Cladonia pseudopityrea Vain. - Ca: Burgaz \& Ahti (2009); H: Burgaz \& Ahti (2009).

Cladonia pulvinella S. Hammer - Al: Burgaz \& Ahti (2009); J: Burgaz \& Ahti (2009); Ma: Burgaz \& Ahti (2009); Se: Burgaz \& Ahti (2009).

Cladonia pyxidata (L.) Hoffm. -Al: Colmeiro (1867), Egea \& Llimona (1981b, 1994); Ca: Fos (1998), Burgaz \& Ahti (2009); Co: Burgaz \& Ahti (2009); Gr: Colmeiro (1867), Sampaio (1917), Burgaz \& Ahti (2009); H: Rowe et al. (1982), Rowe \& Egea (1987a); J: Colmeiro (1867), Aragón et al. (2006a), Aragón \& Rico (1997), Burgaz \& Ahti (2009); Ma: Colmeiro (1867), Burgaz \& Ahti (2009).

Cladonia ramulosa (With.) J. R. Laundon - Ca: Burgaz \& Ahti (2009); Co: Burgaz \& Ahti (2009); H: Rowe et al. (1982), Hernández Gallego \& Muñoz Reinoso (2005), Burgaz \& Ahti (2009); J: Aragón et al. (2006a); Se: Burgaz \& Ahti (2009).

Cladonia rangiformis Hoffm. - Al: Navás (1904), Egea \& Llimona (1994), Alonso \& Egea (1996), Burgaz \& Ahti (2009); Ca: Sampaio (1917), Rowe \& Egea (1987b), Barreno et al. (1988), Rowe et al. (1996), Burgaz \& Ahti (2009); Co: Navás (1904), Burgaz \& Ahti (2009); Gr: Alonso \& Egea (1996), Burgaz \& Ahti (2009); H: Rowe et al. (1982), Hernández Gallego \& Muñoz Reinoso (2005), Burgaz \& Ahti (2009); J: Aragón \& Rico (1997), Burgaz \& Ahti (2009); Ma: Burgaz \& Ahti (2009); Se: Rowe \& Egea (1987a), Burgaz \& Ahti (2009).

Cladonia squamosa (Scop.) Hoffm. - Ca: Burgaz \& Ahti (2009); J: Aragón et al. (2006a), Aragón \& Rico (1997), Burgaz \& Ahti (2009).

Cladonia subcervicornis (Vain.) Kernst. - Ca: Burgaz \& Ahti (2009).

Cladonia subrangiformis (L.) Scriba ex Sandst. - Al: Casares \& Gutiérrez (1993), Gutiérrez \& Casares (1994), Martínez-Sánchez et al. (1994), Alonso \& Egea (1996), Burgaz \& Ahti (2009); Gr: 
Alonso \& Egea (1996), Burgaz \& Ahti (2009); J: Burgaz \& Ahti (2009).

Cladonia subturgida Samp. - Al: Burgaz \& Ahti (2009); Co: Burgaz \& Ahti (2009), Pino-Bodas et al. (2012b); Gr: Burgaz \& Ahti (2009), Pino-Bodas et al. (2012b); H: Burgaz \& Ahti (2009), Pino-Bodas et al. (2012b); J: Burgaz \& Ahti (2009), Pino-Bodas et al. (2012b); Ma: Burgaz \& Ahti (2009); Se: Burgaz \& Ahti (2009), Pino-Bodas et al. (2012b).

Cladonia subulata (L.) Weber ex F. H. Wigg. - Ca: Burgaz \& Ahti (2009); Co: Burgaz \& Ahti (2009); J: Aragón et al. (2006a); H: Rowe et al. (1982); Se: Burgaz \& Ahti (2009).

Cladonia symphycarpa (Flörke) Fr. - Gr: Burgaz \& Ahti (2009); H: Rowe et al. (1982).

Clauzadea chondrodes (A. Massal.) Clauzade \& Cl. Roux - Al: Meyer (2002); Ca: Meyer (2002); Gr: Meyer (2002).

Clauzadea immersa (Hoffm.) Hafellner \& Bellem. - Al: Colmeiro (1867), Alonso \& Egea (1996), Meyer (2002); Ca: Colmeiro (1867), Casares \& Rowe (1988), Alonso \& Egea (1996), Ariño et al. (1997); Gr: Colmeiro (1867), Casares \& Llimona $(1986,1989)$, Alonso \& Egea (1996), Meyer (2002); Ma: Colmeiro (1867).

Clauzadea metzleri (Körb.) Clauzade \& Cl. Roux - Gr: Casares \& Llimona (1989).

Clauzadea monticola (Ach.) Hafellner \& Bellem. - Al: Gutiérrez \& Casares (1994); Gr: Sampaio (1917), Casares \& Llimona (1989); J: Meyer (2002);

Coccocarpia erythroxyli (Spreng.) Swinscow \& Krog - Al: Egea \& Llimona (1994), Pérez Valcárcel et al. (2010); Gr: Colmeiro (1867).

Collema auriforme (With.) Coppins \& J. R. Laundon - Ca: Rowe et al. (1996), Ariño et al. (1997), Carvalho (2012); *Co: MACB 103291; Gr: Casares (1988), Casares \& Llimona (1986, 1989), Carvalho (2012); H: Carvalho (2012); J: Degelius (1954), Carvalho (2012); Ma: Martín-Osorio (1988), Rowe et al. (1996), Carvalho (2012).

Collema callopismum A. Massal. - Ma: Carvalho (2012).

Collema coccophorum Tuck. - Al: Casares \& Gutiérrez (1993), Gutiérrez \& Casares (1994), Guerra et al. (1995), Carvalho (2012); Gr: Casares \& Gutiérrez (1993), Carvalho (2012).

Collema conglomeratum Hoffm. - Gr: Colmeiro (1867), Carvalho (2012).

Collema crispum (Huds.) Weber ex F. H. Wigg. - Al: Alonso \& Egea (1996), Carvalho (2012); Ca: Colmeiro (1867), Degelius (1954), Alonso \& Egea (1996), Carvalho (2012); Gr: Colmeiro (1867), Degelius (1954), Alonso \& Egea (1996), Carvalho (2012); H: Carvalho (2012); J: Degelius (1954), Carvalho (2012); Ma: Degelius (1954), Herrera et al. (2007), Carvalho (2012); Se: Lázaro Ibiza (1898a), Degelius (1954), Puertas et al. (1994), Ariño et al. (1997), Carvalho (2012).

Collema crispum var. metzleri (Arnold) Degel. - Ca: Ariño et al. (1995), Carvalho (2012).

Collema cristatum (L.) Weber var. cristatum - Al: Casares \& Gutiérrez (1993), Gutiérrez \& Casares (1994), Carvalho (2012); Ca: Degelius (1954), Casares \& Rowe (1988), Rowe et al. (1996), Carvalho (2012); Co: Carvalho (2012); Gr: Colmeiro (1867), Degelius (1954), Casares (1988), Casares \& Llimona $(1986,1989)$ Carvalho (2012); J: Degelius (1954), Aragón \& Rico (1997), Carvalho (2012); Ma: Degelius (1954), Carvalho (2012); Se: Carvalho (2012).

Collema cristatum var. marginale (Huds.) Degel. - Ca: Carvalho (2012); Ma: Carvalho (2012); J: Carvalho (2012).

Collema fasciculare (L.) Weber - Ca: Rowe et al. (1996), Carvalho (2012); Gr: Carvalho (2012); J: Degelius (1954), Carvalho
(2012); Ma: Rowe et al. (1996), Seaward \& Arvidson (1997), Carvalho (2012).

Collema flaccidum (Ach.) Ach. - Ca: Fos (1998), Carvalho (2012); Gr: Carvalho (2012); J: Degelius (1954); Aragón \& Rico (1997), Carvalho (2012); Ma: Seaward \& Arvidson (1997), Carvalho (2012).

Collema fragile Taylor - Ca: Degelius (1954), Carvalho (2012); Ma: Degelius (1954), Carvalho (2012).

Collema fragrans (Sm.) Ach. - *Co: MACB 103903; J: Carvalho (2012); Ma: Rowe et al. (1996), Carvalho (2012).

Collema furfuraceum (Arnold) Du Rietz - Ca: Degelius (1954), Barreno et al. (1988), Fos (1998), Hernández Gallego et al. (1998), Carvalho (2012); Co: Carvalho (2012); Gr: Carvalho (2012); H: Carvalho (2012); J: Degelius (1954), Aragón et al. (2006a), Aragón \& Rico (1997), Carvalho (2012); Ma: Degelius (1954), Rowe et al. (1996), Carvalho (2012).

Collema fuscovirens (With.) J. R. Laundon - Ca: Colmeiro (1867), Carvalho (2012); *Co: MACB 103297; Gr: Degelius (1954), Carvalho (2012); J: Degelius (1954), Aragón \& Rico (1997), Carvalho (2012); Ma: Degelius (1954), Carvalho (2012).

Collema glebulentum (Nyl. ex Cromb.) Degel. - Gr: Carvalho (2012).

Collema italicum B. de Lesd. - Ca: Carvalho (2012); Ma: Carvalho (2012).

Collema latzelii Zahlbr. - Ca: Degelius (1954), Carvalho (2012); Ma: Degelius (1954), Carvalho (2012).

Collema ligerinum (Hy) Harm. - J: Degelius (1954), Aragón \& Rico (1997), Carvalho (2012); Ma: Carvalho (2012).

Collema limosum (Ach.) Ach. - Ma: Seaward \& Arvidson (1997), Carvalho (2012).

Collema multipartitum Sm. - Gr: Degelius (1954), Carvalho (2012); J: Degelius (1954), Carvalho (2012); Ma: Degelius (1954), Carvalho (2012).

Collema multipunctum Degel. - Ca: Degelius (1954), Fos (1998), Carvalho (2012); Ma: Carvalho (2012).

Collema nigrescens (Huds.) DC. - Al: Carvalho (2012); Ca: Colmeiro (1867), Degelius (1954), Barreno et al. (1988), Rowe et al. (1996), Fos (1998), Carvalho (2012); Gr: Fos (1998), Carvalho (2012); J: Degelius (1954), Carvalho (2012); Ma: Colmeiro (1867), Rowe et al. (1996), Fos (1998), Carvalho (2012).

Collema occultatum Bagl. - Ca: Carvalho (2012); J: Degelius (1954), Aragón \& Rico (1997), Carvalho (2012); Ma: Degelius (1954), Rowe et al. (1996), Carvalho (2012).

Collema parvum Degel. - Ca: Carvalho (2012); J: Degelius (1954), Carvalho (2012).

Collema polycarpon Hoffm. - Ca: Degelius (1954), Casares \& Rowe (1988); Gr: Degelius (1954), Casares \& Llimona (1989), Carvalho (2012); J: Degelius (1954), Carvalho (2012); Ma: Degelius (1954), Seaward \& Arvidson (1997), Carvalho (2012).

Collema polycarpon subsp. corcyrense (Arnold) Pišút - Ca: Carvalho (2012); Gr: Casares \& Llimona (1989); Carvalho (2012); J: Carvalho (2012); Ma: Carvalho (2012).

Collema ryssoleum (Tuck.) A. Schneid. -Al: Egea \& Llimona (1981a, 1987), Llimona \& Egea (1985), Carvalho (2012); Ca: Degelius (1954), Barreno et al. (1988), Rowe \& Egea (1988), Carvalho (2012); Ma: Degelius (1954), Seaward \& Arvidson (1997), Carvalho (2012); Se: Rowe \& Egea (1985a, b), Carvalho (2012).

Collema subflaccidum Degel. - Ca: Fos (1998); Ma: Rowe et al. (1996), Fos (1998).

Collema subfurvum (Müll. Arg.) Degel. - J: Degelius (1954).

Collema subnigrescens Degel. - Ca: Colmeiro (1867), Degelius (1954), Rowe et al. (1996), Fos (1998), Carvalho (2012); Co: Car- 
valho (2012); H: Carvalho (2012); J: Degelius (1954), Aragón \& Rico (1997), Carvalho (2012); Ma: Degelius (1954), Martín-Osorio (1988), Rowe et al. (1996), Seaward \& Arvidson (1997), Carvalho (2012).

Collema substellatum H. Magn. - Gr: Hansen (1993).

Collema tenax (Sw.) Ach. - Al: Degelius (1954), Casares \& Gutiérrez (1993), Martínez-Sánchez et al. (1994), Carvalho (2012); Ca: Degelius (1954), Ariño et al. (1997), Carvalho (2012); *Co: MACB 103299; Gr: Degelius (1954), Casares (1988), Casares \& Llimona (1986, 1989), Martínez-Sánchez et al. (1994), Alonso \& Egea (1996), Carvalho (2012); H: Carvalho (2012); J: Degelius (1954), Carvalho (2012); Ma: Colmeiro (1867), Degelius (1954), Carvalho (2012); Se: Carvalho (2012).

Collema tenax var. corallinum (A. Massal.) Degel. -Al: Gutiérrez \& Casares (1994); Ca: Rowe et al. (1996).

Collema undulatum Laurer ex Flot. - Ca: Casares \& Rowe (1988), Carvalho (2012); *Co: MACB 103908; Gr: Degelius (1954), Carvalho (2012); J: Degelius (1954), Aragón \& Rico (1997), Carvalho (2012); Ma: Otálora et al. (2008), Carvalho (2012).

Collema undulatum var. granulosum Degel. - Gr: Casares \& Llimona $(1986,1989)$.

Coniocybe furfuracea (L.) Ach. -Al: Egea \& Llimona (1981a).

\#Corticifraga fuckelii (Rehm) D. Hawksw. \& R. Sant. - J: Martínez \& Hafellner (1998); Ma: Martínez (1999).

\#Corticifraga peltigerae (Fuckel) D. Hawksw. \& R. Sant. - J: Martínez \& Hafellner (1998), Martínez (1999).

Coscinocladium gaditanum (Clemente) A. Crespo et al. (sub Variolaria gaditana Clem. Ens. Var. Vid.) - Ca: Kunze (1846), Colmeiro (1867), Crespo et al. (2004).

Cresponea premnea (Ach.) Egea \& Torrente - Ca: Torrente \& Egea (1989a).

Cyphelium inquinans (Sm.) Trevis. - J: Sarrión et al. (1999), Aragón et al. (2006a), Muñiz \& Hladun (2011).

Cyphelium lecideinum (Nyl.) Trevis. - Ca: Rowe \& Egea (1988); Se: Sarrión et al. (1999).

Cyphelium tigillare (Ach.) Ach. - Gr: Colmeiro (1867), Muñiz \& Hladun (2011); J: Sarrión et al. (1999), Aragón et al. (2004c, 2006a), Muñiz \& Hladun (2011).

\#Dacampia rufescentis (Vouaux) D. Hawksw. - J: Martínez \& Hafellner (1998), Martínez (1999); Ma: Martínez (1999).

\#Dactylospora parasitica (Flörke) Zopf - Ma: Rowe et al. (1996).

\#Dactylospora saxatilis (Schaer.) Hafellner - Al: Egea \& Llimona (1981a, 1983); Ca: Rowe \& Egea (1986, 1988); Egea \& Rowe (1987); Se: Rowe \& Egea (1985b).

Degelia atlantica (Degel.) P. M. Jørg. \& P. James - J: Martínez \& Aragón (1994), Aragón \& Rico (1997), Carballal et al. (2010b); Ma: Martín-Osorio (1988), Martínez \& Aragón (1994).

Degelia plumbea (Lightf.) P. M. Jørg. \& P. James - Ca: Colmeiro (1867), Barreno et al. (1988), Martínez \& Aragón (1994), Rowe et al. (1996), Fos (1998), Hernández Gallego et al. (1998), Carballal et al. (2010b); Gr: Colmeiro (1867), Martínez \& Aragón (1994); J: Aragón et al. (2006a), Aragón \& Rico (1997), Carballal et al. (2010b); Ma: Abbayes (1945), Martín-Osorio (1988), Rowe et al. (1996), Martínez \& Aragón (1994), Seaward \& Arvidson (1997), Carballal et al. (2010b).

Dendrographa decolorans (Turner \& Borrer ex Sm.) Ertz \& Tehler Ca: Colmeiro (1867), Rowe et al. (1996), Fos (1998); Gr: Colmeiro (1867); H: Fos (1998); Ma: Colmeiro (1867).

Dermatocarpon decipiens (A. Massal.) Dalla Torre \& Sarnth. - Al: Egea \& Llimona (1981a, 1987); Gr: Egea et al. (1982).
Dermatocarpon luridum (Dill. ex With.) J. R. Laundon - Ca: Colmeiro (1867); Gr: Colmeiro (1867), Egea et al. (1982), Pereira et al. (1987); Ma: Colmeiro (1867).

Dermatocarpon meiophyllizum Vain. - Gr: Casares \& Llimona (1982), Pereira et al. (1987).

Dermatocarpon miniatum var. cirsodes (Ach.) Zahlbr. - Al: Egea \& Llimona (1981a); Gr: Egea et al. (1982), Casares (1988).

Dermatocarpon miniatum var. complicatissimum (Nyl.) Lettau - Gr: Egea et al. (1982); J: Aragón \& Rico (1997).

Dermatocarpon miniatum var. complicatum (Lightf.) Th. Fr. - Gr: Colmeiro (1867), Egea et al. (1982).

Dermatocarpon miniatum (L.) W. Mann var. miniatum - Al: Alonso \& Egea (1996); Ca: Colmeiro (1867), Casares \& Rowe (1988); Gr: Colmeiro (1867), Werner (1979), Casares \& Llimona (1984, 1986), Casares (1988, 1992); Ma: Colmeiro (1867).

Dermatocarpon miniatum var. umbilicatum (Schaer.) Vain. - Gr: Casares \& Llimona (1982).

Dermatocarpon rivulorum (Arnold) Dalla Torre \& Sarnth. - Gr: Egea et al. (1982).

Dermatocarpon trachyticum (Hazsl.) Vain. - Gr: Casares \& Llimona (1984, 1986).

\#Didymellopsis pulposi (Zopf) Grube \& Hafellner - Ma: López de Silanes et al. (2009).

Dimelaena oreina (Ach.) Norman -Al: Egea \& Llimona (1983, 1987); *Co: MACB 103158; Gr: Colmeiro (1867), Prieto \& Espinosa (1971), Egea et al. (1982).

Dimelaena radiata (Tuck.) Hale \& W. L. Culb. - Al: Llimona (1975), Egea \& Llimona (1981b, 1983, 1987, 1994), Rico et al. (2003); Ma: Herrera et al. (2007).

Dimerella pineti (Schrad.) Vězda - Ca: Colmeiro (1867), Rowe et al. (1996), Fos (1998), Paz Bermúdez (2004).

Diploicia canescens (Dicks.) A. Massal. - Ca: Colmeiro (1867), Silvestre \& Rowe (1982).

Diploicia subcanescens (Werner) Hafellner \& Poelt - Al: Llimona (1975), Egea \& Llimona (1981b, 1983, 1984, 1987, 1994), Alonso \& Egea (1996); Gr: Alonso \& Egea (1996); Ma: Alonso \& Egea (1996), Herrera et al. (2007).

Diploschistes actinostomus (Ach.) Zahlbr. - Al: Egea \& Llimona (1981a, b, 1983, 1987, 1994); *Co: 106706; Ca: Rowe \& Egea (1986, 1988); Se: Rowe \& Egea (1985b).

Diploschistes caesioplumbeus (Nyl.) Vain. - Se: Rowe \& Egea (1987a).

Diploschistes diacapsis (Ach.) Lumbsch - Al: Llimona (1974), Egea \& Llimona (1981a, 1987), Casares \& Gutiérrez (1993), Martínez-Sánchez et al. (1994), Alonso \& Egea (1996), Pintado et al. (2005), Souza-Egipsy et al. (2002), Gutiérrez \& Casares (1994); Gr: Casares \& Llimona (1989), Casares \& Gutiérrez (1993), Martínez-Sánchez et al. (1994), Alonso \& Egea (1996); Ma: Colmeiro (1867), Seaward \& Arvidson (1997), Mottershead \& Lucas (2000).

Diploschistes euganeus (A. Massal.) Zahlbr. - Ca: Rowe \& Egea (1986); Se: Rowe \& Egea (1985b).

Diploschistes gypsaceus (Ach.) Zahlbr. -Al: Egea \& Llimona (1981a, 1987); Gr: Casares \& Llimona (1989).

Diploschistes muscorum (Scop.) R. Sant. -Al: Egea \& Llimona (1994), Alonso \& Egea (1996); Ca: Colmeiro (1867), Sampaio (1917); Gr: Colmeiro (1867), Casares \& Llimona (1989); H: Rowe et al. (1982), Rowe \& Egea (1987a); J: Colmeiro (1867); Ma: Rowe et al. (1996).

Diploschistes ocellatus var. almeriensis Llimona -Al: Llimona (1974), Casares \& Gutiérrez (1993), Martínez-Sánchez et al. (1994), Gu- 
tiérrez \& Casares (1994), Guerra et al. (1995), Winka et al. (1998), Martín et al. (2000).

Diploschistes scruposus (Schreb.) Norm. -Al: Egea \& Llimona (1981b, 1983, 1987), Llimona \& Egea (1985); Ca: Colmeiro (1867), Rowe \& Egea (1986, 1988), Egea \& Rowe (1987), Rowe et al. (1996), Fos (1998); *Co: MACB 103402; Gr: Colmeiro (1867), Egea et al. (1982), Casares \& Llimona (1989); H: Rowe et al. (1982), Rowe \& Egea (1987a), Hernández Gallego \& Muñoz Reinoso (2005); J: Colmeiro (1867); Ma: Colmeiro (1867), Rowe et al. (1996); Se: Colmeiro (1867), Barras (1899), Lázaro Ibiza (1898a), Rowe \& Egea (1985a, b, 1987a).

Diplotomma alboatrum (Hoffm.) Flot. - Ca: Rowe et al. (1996); Ma: Martín-Osorio (1988), Rowe et al. (1996).

Diplotomma ambiguum (Ach.) Flagey - Al: Egea \& Llimona (1994); Gr: Colmeiro (1867).

Diplotomma chlorophaeum (Hepp ex Leight.) Szatala - Al: Egea \& Llimona (1981a, 1983, 1994); Ca: Rowe \& Egea (1986).

Diplotomma hedinii (H. Magn.) P. Clerc \& Cl. Roux -Al: Egea \& Llimona (1981a, 1987, 1994), Casares \& Gutiérrez (1993), Gutiérrez \& Casares (1994), Alonso \& Egea (1996); Ca: Casares \& Rowe (1988), Alonso \& Egea (1996), Rowe et al. (1996); *Co: MACB 103403; Gr: Casares (1988, 1992), Casares \& Llimona (1986, 1989).

Diplotomma venustum (Körb.) Körb. - Al: Egea \& Llimona (1981b); Ca: Casares \& Rowe (1988); Gr: Casares (1988), Casares \& Llimona (1986, 1989); Ma: Nordin (2000), Herrera et al. (2007).

Dirina ceratoniae (Ach.) Fr. - Ma: Herrera et al. (2007).

Dirina massiliensis Durieu \& Mont. -Al: Egea \& Llimona (1994), Gutiérrez \& Casares (1994), Alonso \& Egea (1996); Ca: Egea \& Rowe (1987); Gr: Casares \& Llimona (1989), Alonso \& Egea (1996); Ma: Herrera et al. (2007).

Dirina massiliensis f. sorediata (Müll. Arg.) Tehler -Al: Egea \& Llimona (1984, 1994); Ca: Egea \& Rowe (1987); Gr: Casares \& Llimona (1984); Se: Ariño et al. (1997).

Dirina massiliensis. f. schistosa (Bagl.) Durieu et Mont-Al: Egea \& Llimona (1981b); Ca: Rowe \& Egea (1986).

Dirina paradoxa subsp. africana (Fée) Tehler - Ma: Herrera et al. (2007).

Endocarpon pusillum Hedw. - Al: Casares \& Gutiérrez (1993), Gutiérrez \& Casares (1994), Alonso \& Egea (1996); *Co: MACB 103404; Gr: Casares \& Llimona (1984), Martínez-Sánchez et al. (1994); J: Aragón \& Rico (1997); Se: Ariño et al. (1997).

Endohyalina insularis (Arnold) Giralt, Van den Boom \& Elix - Al: Egea (1985).

Enterographa hutchinsiae (Leight.) A. Massal. - Ca: Rowe \& Egea (1986).

Enterographa zaborskiana (M. Choisy \& Werner) Egea \& Torrente Ca: Torrente \& Egea (1989a)

Evernia prunastri (L.) Ach. - Ca: Colmeiro (1867), Sampaio (1917), Abbayes (1945), Rowe \& Egea (1986), Barreno et al. (1988), Rowe et al. (1996), Hernández Gallego et al. (1998), Fos (1998), Diederich \& Etayo (2000); Co: Diederich \& Etayo (2000); Gr: Colmeiro (1867), Fos (1998); H: Colmeiro (1867), Rowe et al. (1982), Fos (1998); J: Lázaro Ibiza (1898a), Sampaio (1917), Aragón \& Rico (1997), Aragón et al. (2006a); Ma: Colmeiro (1867), Sampaio (1917), Martín-Osorio (1988), Rowe et al. (1996), Fos (1998); Se: Barras (1896, 1899), Silvestre \& Rowe (1982).

Farnoldia jurana (Schaer.) Hertel subsp. jurana - Gr: Casares \& Llimona $(1986,1989)$.

Flavoparmelia caperata (L.) Hale - Ca: Colmeiro (1867), Sampaio
(1917), Rowe \& Egea (1986), Egea \& Rowe (1987), Barreno et al. (1988), Rowe et al. (1996), Fos (1998), Hernández Gallego et al. (1998); Co: Navás (1901); Gr: Colmeiro (1867); H: Rowe et al. (1982), Fos (1998); J: Aragón \& Rico (1997); Ma: Colmeiro (1867), Martín-Osorio (1988), Rowe et al. (1996), Fos (1998); Se: González-Fragoso (1883), Barras (1896), Lázaro Ibiza (1898b), Navás (1901), Silvestre \& Rowe (1982).

Flavoparmelia caperata var. laevissima (Gyeln.) Ozenda \& Clauzade - Ma: Rowe et al. (1996).

Flavoparmelia soredians (Nyl.) Hale - Ca: Sampaio (1917), Barreno et al. (1988), Rowe et al. (1996), Fos (1998); *Co: MACB 103412; H: Fos (1998).

Flavopunctelia flaventior (Stirt.) Hale - *Co: MACB 103910; J: Aragón \& Rico (1997), Aragón et al. (2006a).

Flavoplaca citrina (Hoffm.) Arup et al. - Al: Casares \& Gutiérrez (1993), Gutiérrez \& Casares (1994), Alonso \& Egea (1996); Ca: Sampaio (1917), Fos (1998), Ariño et al. (1995, 1997), Ariño \& Saiz-Jimenez (1996), Rowe et al. (1996); Co: Colmeiro (1867); Gr: Egea (1984), Casares \& Llimona (1986, 1989); J: Aragón et al. (2006a); Ma: Herrera et al. (2007); Se: Puertas et al. (1994), Ariño et al. (1997).

Flavoplaca granulosa (Müll. Arg.) Arup et al. - Gr: Egea (1984); Ma: Seaward \& Arvidson (1997).

Flavoplaca microthallina (Wedd.) Arup et al. - Ma: Herrera et al. (2007).

Fulgensia poeltii Llimona-Al: Llimona (1974), Casares \& Gutiérrez (1993), Guerra et al. (1995); Gr: Casares \& Gutiérrez (1993).

Fuscidea cyathoides (Ach.) V. Wirth \& Vězda - Ca: Crespo \& Bueno (1984), Rowe \& Egea (1986), Egea \& Rowe (1987), Barreno et al. (1988).

Fuscidea lygaea (Ach.) V. Wirth \& Vězda - Ca: Rowe \& Egea (1986, 1987b), Rowe et al. (1996); Se: Rowe \& Egea (1987a).

Fuscopannaria ignobilis (Anzi) P. M. Jørg. - Ca: Paz-Bermúdez et al. (2010a); J: Aragón \& Rico (1997), Paz-Bermúdez et al. (2010a); Ma: Seaward \& Arvidson (1997), Paz-Bermúdez et al. (2010a).

Fuscopannaria leucosticta (Tuck.) P. M. Jørg. - Ca: Rowe et al. (1996); Ma: Martín-Osorio (1988).

Fuscopannaria mediterranea (Tav.) P. M. Jørg. - Ca: Martínez \& Aragón (1994), Rowe et al. (1996), Fos (1998), Calatayud et al. (2000), Paz-Bermúdez et al. (2010a); *Co: MACB 103112; J: Martínez \& Aragón (1994), Aragón \& Rico (1997), Aragón et al. (2004c, 2006a), Paz-Bermúdez et al. (2010a); Ma: Rowe et al. (1996), Seaward \& Arvidson (1997), Fos (1998), Paz-Bermúdez et al. (2010a); Se: Paz-Bermúdez et al. (2010a).

Fuscopannaria olivacea (P. M. Jørg.) P. M. Jørg. - Ca: Rowe et al. (1996), Fos (1998), Paz-Bermúdez et al. (2010a); J: Aragón \& Rico (1997), Aragón et al. (2004c), Paz-Bermúdez et al. (2010a); Ma: Rowe et al. (1996), Seaward \& Arvidson (1997).

Gloeoheppia turgida (Ach.) Gyeln. -Al: Egea (1989), Alonso \& Egea (1996); Gr: Casares \& Llimona (1989), Alonso \& Egea (1996).

Glyphopeltis ligustica (B.de Lesd.) Timdal -Al: Llimona \& Egea (1985), Egea et al. (1995); Ca: Rowe \& Egea (1988), Egea et al. (1995); Gr: Timdal (1988), Egea et al. (1995); Se: Egea et al. (1995).

\#Guignardia insularis (A. Massal.) Keissl. - Gr: Casares \& Llimona (1984, 1986).

Gyalecta derivata (Nyl.) H. Olivier - Ca: Fos (1998), López de Silanes \& Álvarez (2004); Gr: Fos (1998).

Gyalecta jenensis (Batsch) Zahlbr. - Ma: López de Silanes \& Álvarez (2004).

Gyalecta truncigena (Ach.) Hepp - Ca: Rowe et al. (1996). 
Gyalecta ulmi (Sw.) Zahlbr. -Al: López de Silanes \& Álvarez (2004); Ca: López de Silanes \& Álvarez (2004); Ma: Colmeiro (1867).

Gyalolechia desertorum (Huds.) Søchting et al. - Al: Llimona (1974), Casares \& Gutiérrez (1993); Gr: Casares \& Gutiérrez (1993), Martínez-Sánchez et al. (1994); Ma: Seaward \& Arvidson (1997).

Gyalolechia flavorubescens (Huds.) Søchting et al. - Ca: Fos (1998); ${ }^{*}$ Co: MACB 103896; Gr: Colmeiro (1867), Fos (1998); J: Aragón et al. (2004c, 2006a); Ma: Colmeiro (1867), Herrera et al. (2007).

Gyalolechia flavovirescens (Wulfen) Søchting et al. -Al: Egea \& Llimona (1981a, 1987); Gr: Egea (1984), Casares \& Llimona (1989); J: Colmeiro (1867); Ma: Herrera et al. (2007); Se: Barras (1896).

Gyalolechia fulgens (Sw.) Søchting et al. -Al: Alonso \& Egea (1996); Ca: Colmeiro (1867), Casares \& Rowe (1988); Co: Colmeiro (1867); Gr: Colmeiro (1867), Casares \& Llimona (1989), Alonso \& Egea (1996); Ma: Colmeiro (1867); Se: Barras (1896, 1899), Silvestre \& Rowe (1982).

Gyalolechia fulgida (Nyl.) Søchting et al. - Al: Casares \& Gutiérrez (1993), Gutiérrez \& Casares (1994), Martínez-Sánchez et al. (1994), Alonso \& Egea (1996); Ca: Rowe et al. (1996); Gr: Sampaio (1917), Casares (1988), Casares \& Llimona (1989), Casares \& Gutiérrez (1993), Martínez-Sánchez et al. (1994), Alonso \& Egea (1996).

Gyalolechia klementii (Kalb) Søchting et al. - Ca: Sa del Pinar de Grazalema (Kalb 1970).

Gyalolechia subbracteata (Nyl.) Søchting et al. - Al: Casares \& Gutiérrez (1993), Gutiérrez \& Casares (1994), Martínez-Sánchez et al. (1994); Gr: Casares \& Gutiérrez (1993), Martínez-Sánchez et al. (1994), Alonso \& Egea (1996)

Haematomma ochroleucum (Neck.) J. R. Laundon - Ca: Colmeiro (1867); Gr: Colmeiro (1867).

Haematomma ochroleucum var. porphyrium (Pers.) J. R. LaundonCa: Rowe \& Egea $(1986,1988)$.

\#Hawksworthiana peltigericola (D. Hawksw.) U. Braun-Ma: Martínez \& Hafellner (1998), Martínez (1999).

Heppia despreauxii (Mont.) Tuck. -Al: Egea (1989); Ca: Egea (1989); Gr: Egea (1989), Alonso \& Egea (1996); Ma: Seaward \& Arvidson (1997).

Heppia solorinoides (Nyl.) Nyl. -Al: Egea (1989), Casares \& Gutiérrez (1993), Gutiérrez \& Casares (1994), Alonso \& Egea (1996); Gr: Casares \& Llimona (1983, 1989), Alonso \& Egea (1996).

Heterodermia leucomelos (L.) Poelt - Ca: Colmeiro (1867), Abbayes (1945), Barreno et al. (1988), Rowe et al. (1996); Ma: Fos (1998).

Heterodermia obscurata (Nyl.) Trevis - Al: Egea (1989); Ca: Barreno et al. (1988), Fos (1998).

Heteroplacidium contumescens (Nyl.) Breuss - Al: Egea \& Llimona (1981a, 1994), Llimona \& Egea (1985), Prieto et al. (2010); Gr: Prieto et al. (2010); Ma: Prieto et al. (2010).

Heteroplacidium divisum (Zahlbr.) Breuss -Al: Alonso \& Egea (1996), Prieto et al. (2010); Gr: Alonso \& Egea (1996), Prieto et al. (2010).

Heteroplacidium imbricatum (Nyl.) Breuss - Al: Egea \& Llimona (1981b); Ca: Prieto et al. (2010); Gr: Casares \& Llimona (1984), Prieto et al. (2010); J: Prieto et al. (2010).

Heteroplacidium fusculum (Nyl.) Gueidan \& Cl. Roux - Ma: Herrera et al. (2007).

Heteroplacidium phaeocarpoides (Nyl.) Breuss - Al: Prieto et al. (2010).

Hydropunctaria amphibia (Clemente) Orange - Gr: Casares \& Llimona (1984); Ma: Herrera et al. (2007).
Hydropunctaria rheitrophila (Zsch.) Keller et al. - Gr: Pereira et al. (1987); H: Valcárcel \& López de Silanes (2010).

Hymenelia epulotica (Ach.) Lutzoni - Ca: Casares \& Rowe (1988), Rowe et al. (1996); Gr: Casares \& Llimona (1983, 1986, 1989).

Hymenelia melanocarpa (Kremp.) Arnold - Ca: Casares \& Rowe (1988).

Hyperphyscia adglutinata (Flörke) H. Mayrhofer \& Poelt - Ca: Rowe et al. (1996); Ma: Seaward \& Arvidson (1997).

Hypocenomyce anthracophila (Nyl.) P. James \& Gotth. Schneid. - J: Aragón et al. (2006a).

Hypocenomyce scalaris (Ach. ex Lilj.) M. Choisy - J: Sarrión \& Burgaz (1995), Aragón et al. (2004c, 2006a).

Hypogymnia bitteri (Lynge) Ahti - Ca: Rowe et al. (1996).

Hypogymnia farinacea Zopf - J: Aragón \& Rico (1997), Aragón et al. (2006a).

Hypogymnia physodes (L.) Nyl. - Ca: Abbayes (1945), Barreno et al. (1988), Fos (1998); H: Fos (1998); J: Aragón \& Rico (1997), Aragón et al. (2006a); Ma: Colmeiro (1867), Martín-Osorio (1988), Rowe et al. (1996).

Hypogymnia tubulosa (Schaer.) Hav. - Ca: Abbayes (1945), Egea \& Rowe (1987), Barreno et al. (1988), Rowe et al. (1996); H: Rowe et al. (1982), Fos (1998); J: Aragón \& Rico (1997), Aragón et al. (2004c, 2006a); Ma: Martín-Osorio (1988), Rowe et al. (1996), Fos (1998).

\#Illosporiopsis christiansenii (B. L. Brady \& D. Hawksw.) D. Hawksw. - Ma: Rowe et al. (1996).

Immersaria athroocarpa (Ach.) Rambold \& Pietschm. - Al: Egea \& Llimona (1981a, 1987).

Imshaugia aleurites (Ach.) S. L. F.Mey. - J: Aragón \& Rico (1997), Aragón et al. (2006a).

Ingaderia troglodytica Feige \& Lumbsch-Al: Alonso \& Egea (1996).

Ingvariella bispora (Bagl.) Guderley \& Lumbsch - Ca: Rowe \& Egea (1987b), Rowe et al. (1996).

\#Kiliasia athallina (Hepp) Hafellner-Gr: Casares \& Llimona (1989).

Koerberia biformis A. Massal. - Ca: Rowe et al. (1996), Burgaz (2011a, b); *Co: MACB 103108; J: Aragón \& Rico (1997), Aragón \& Sarrión (2003); Ma: Seaward (1983), Rowe et al. (1996), Burgaz (2011a, b); Se: Burgaz (2011a, b).

Koerberia sonomensis (Tuck.) Henssen - J: Burgaz (2011a, b).

Lasallia pustulata (L.) Mérat - Ca: Colmeiro (1867), Rowe \& Egea (1986, 1988), Egea \& Llimona (1987), Egea \& Rowe (1987); Co: Colmeiro (1867); Gr: Colmeiro (1867); J: Sarrión \& Burgaz (1995); H: Rowe et al. (1982), Rowe \& Egea (1987a); Se: Rowe \& Egea (1985a, 1987a).

Lecanactis abietina (Ehrh. ex Ach.) Körb. - Ca: Rowe \& Egea (1986), Egea \& Rowe (1987); Gr: Colmeiro (1867).

Lecanactis plocina (Ach.) A. Massal. - Ca: Rowe \& Egea (1986), Egea \& Rowe (1987).

Lecania aipospila (Wahlenb.) Th. Fr. - Ma: Herrera et al. (2007).

Lecania arenaria (Anzi) Flagey -Al: Egea \& Llimona (1981a, 1987).

Lecania atrynoides M. Knowles - Ma: Herrera et al. (2007).

Lecania cuprea (A. Massal.) Van den Boom \& Coppins - Ma: Rowe et al. (1996); Se: Ariño et al. (1997).

Lecania cyrtella (Ach.) Th. Fr. - Ca: Barreno et al. (1988); Ma: Rowe et al. (1996), Herrera et al. (2007).

Lecania erysibe (Ach.) Mudd - Ca: Casares \& Rowe (1988); Gr: Casares (1988), Casares \& Llimona (1986, 1989).

Lecania fuscella (Schaer.) A. Massal. - Gr: Fos (1998).

Lecania hutchinsiae (Nyl.) A. L. Sm. - Ma: Herrera et al. (2007).

Lecania koerberiana J. Lahm - J: Aragón et al. (2006a). 
Lecania naegelii (Hepp) Diederich \& Van den Boom - Ca: Rowe et al. (1996), Fos (1998); Gr: Fos (1998); H: Fos (1998); Ma: Rowe et al. (1996).

Lecania nylanderiana A. Massal. - Al: Gutiérrez \& Casares (1994), Alonso \& Egea (1996); Ma: Rowe et al. (1996).

Lecania rabenhorstii (Hepp) Arnold - Gr: Casares \& Llimona (1986, 1989).

Lecania spadicea (Flot.) Zahlbr. - Al: Egea \& Llimona (1994).

Lecania turicensis (Hepp) Müll. -Al: Egea \& Llimona (1994), Gutiérrez \& Casares (1994), Alonso \& Egea (1996); Ca: Ariño et al. (1995, 1997), Ariño \& Saiz-Jimenez (1996), Alonso \& Egea (1996); Gr: Alonso \& Egea (1996); Ma: Herrera et al. (2007); Se: Puertas et al. (1994), Ariño et al. (1997).

Lecanographa lyncea (Sm.) Egea \& Torrente - Ca: Colmeiro (1867), Barreno et al. (1988), Torrente \& Egea (1989a).

Lecanora achariana A. L. Sm. - Gr: Casares \& Llimona (1982).

Lecanora admontensis Zahlbr. - Gr: Casares (1988), Casares \& Llimona (1989).

Lecanora agardhiana Ach. subsp. agardhiana - Gr: Casares \& Llimona $(1986,1989)$.

Lecanora albella (Pers.) Ach. - Ca: Barreno et al. (1988), Rowe et al. (1996), Fos (1998); *Co: MACB 103331; H: Fos (1998); Ma: Fos (1998); Se: Lázaro Ibiza (1898a).

Lecanora albescens (Hoffm.) Branth \& Rostr. - Al: Egea \& Llimona (1981a, b, 1987, 1994), Gutiérrez \& Casares (1994), Alonso \& Egea (1996); Ca: Sampaio (1917), Casares \& Rowe (1988), Ariño et al. (1995, 1997), Rowe et al. (1996); Gr: Colmeiro (1867), Casares \& Llimona (1986, 1989), Alonso \& Egea (1996); Se: Puertas et al. (1994), Ariño et al. (1997).

Lecanora argentata (Ach.) Malme - Ca: Barreno et al. (1988), Rowe et al. (1996), Fos (1998); H: Fos (1998)

Lecanora argopholis (Ach.) Ach. -Al: Egea \& Llimona (1981a, 1987, 1994); Gr: Pereira et al. (1987).

Lecanora caesiosora Poelt -Al: Egea \& Llimona (1983), Egea (1985); Gr: Egea (1985).

Lecanora campestris (Schaer.) Hue - Al: Egea \& Llimona (1981a, b, 1983, 1985, 1987, 1994), Casares \& Gutiérrez (1993), Gutiérrez \& Casares (1994); Ca: Rowe \& Egea $(1986,1988)$, Egea \& Rowe (1987); Gr: Casares \& Llimona (1989), Alonso \& Egea (1996); H: Rowe et al. (1982), Rowe \& Egea (1987a); Se: Barras (1896), Rowe \& Egea (1985a, 1987a).

Lecanora carpinea (L.) Vain. - Ca: Colmeiro (1867); *Co: MACB 103332; J: Aragón et al. (2006a); Ma: Herrera et al. (2007).

Lecanora cenisia Ach. -Al: Egea \& Llimona (1981b, 1987); Gr: Egea et al. (1982)

Lecanora cernohorskyana Clauzade \& Vězda - Al: Egea \& Llimona (1981b).

Lecanora chlarotera Nyl. - Ca: Barreno et al. (1988), Rowe et al. (1996); *Co: MACB 103912; J: Aragón et al. (2004c, 2006a); Ma: Martín-Osorio (1988), Rowe et al. (1996).

Lecanora circumborealis Brodo \& Vitik. - Ca: Fos (1998); Gr: Ibáñez \& Burgaz (1998); H: Fos (1998).

Lecanora coilocarpa (Ach.) Nyl. - Ca: Barreno et al. (1988).

Lecanora concolor Ramond - Gr: Degelius (1966), Prieto \& Espinosa (1971), Egea et al. (1982).

Lecanora conferta (Duby ex Fr.) Grognot - Al: Egea \& Llimona (1981a); Gr: Casares \& Llimona (1989), Se: Barras (1896, 1899).

Lecanora coniferarum Printzen - J: Martínez \& Aragón (2004), Aragón et al. (2006a).

Lecanora conizaeoides Nyl. ex Cromb. - J: Martínez \& Aragón (2004).
Lecanora conizella Nyl. - Ca: Rowe et al. (1996).

Lecanora crenulata Hook. - Al: Casares \& Gutiérrez (1993), Gutiérrez \& Casares (1994); Ca: Colmeiro (1867), Rowe et al. (1996); Gr: Colmeiro (1867), Casares (1988), Casares \& Llimona (1986, 1989).

Lecanora densa (Âliwa \& Wetmore) Printzen - J: Aragón et al. (2006a).

Lecanora dispersa (Pers.) Röhl. -Al: Egea \& Llimona (1981a, b, 1987, 1994), Casares \& Gutiérrez (1993), Gutiérrez \& Casares (1994), Alonso \& Egea (1996); Ca: Rowe \& Egea (1986), Casares \& Rowe (1988), Ariño et al. (1995), Rowe et al. (1996), Fos (1998); Gr: Casares (1988), Casares \& Llimona (1986, 1989); Ma: Herrera et al. (2007); Se: Ariño et al. (1997).

Lecanora dispersa f. pruinosa B. de Lesd. - Gr: Casares \& Llimona (1986), Casares (1988).

Lecanora epanora (Ach.) Ach. - Gr: Egea et al. (1982).

Lecanora expallens Ach. - Ca: Fos (1998); J: Aragón et al. (2006a).

Lecanora frustulosa (Dicks.) Ach. -Al: Egea \& Llimona (1981a, 1983, 1987); Gr: Egea et al. (1982).

Lecanora gangaleoides Nyl.-Al: Egea \& Llimona (1981a, b, 1983, 1987, 1994), Casares \& Gutiérrez (1993), Gutiérrez \& Casares (1994), Alonso \& Egea (1996); Ca: Rowe \& Egea (1986, 1988); Gr: Casares \& Llimona (1982), Egea et al. (1982); H: Rowe \& Egea (1987a).

Lecanora garovaglioi (Körb.) Zahlbr. - Al: Egea \& Llimona (1981a, 1983, 1987); Gr: Egea et al. (1982).

Lecanora hagenii (Ach.) Ach. - Gr: Casares (1988), Casares \& Llimona (1989); J: Aragón et al. (2004c, 2006a).

Lecanora helicopis (Wahlenb. ex Ach.) Ach. - Ma: Herrera et al. (2007).

Lecanora horiza (Ach.) Linds. - Ca: Rowe et al. (1996), Fos (1998); J: Aragón et al. (2004c, 2006a); Ma: Rowe et al. (1996).

Lecanora hybocarpa (Tuck.) Brodo - Ca: Rowe et al. (1996), Fos (1998); Ma: Rowe et al. (1996).

Lecanora incusa (Flot.) Vain. - Gr: Prieto \& Espinosa (1971).

Lecanora intricata (Ach.) Ach. - Ca: Rowe \& Egea (1986), Egea \& Rowe (1987).

Lecanora intumescens (Rebent.) Rabenh. - Ma: Rowe et al. (1996).

Lecanora laatokkensis (Räsänen) Poelt-Al: Egea \& Llimona (1981a, 1987); Ca: Rowe \& Egea (1986).

Lecanora lividocinerea Bagl. - Ca: Barreno et al. (1988), Fos (1998).

Lecanora lojkaeana Szatala -Al: Egea (1985), Egea \& Llimona (1987).

Lecanora meridionalis H. Magn. - *Co: MACB 103913; Gr: Ibáñez \& Burgaz (1998).

Lecanora mughicola Nyl. - J: Martínez \& Aragón (2004).

Lecanora muralis (Schreb.) Rabenh. - Al: Colmeiro (1867), Egea \& Llimona (1981a, b, 1985, 1994, 1997); Ca: Rowe \& Egea (1986, 1987, 1987b, 1988), Rowe et al. (1996); *Co: MACB 103914; Gr: Colmeiro (1867), Sampaio (1917), Werner (1979), Egea et al. (1982), Casares \& Llimona (1989); H: Rowe \& Egea (1987a); J: Colmeiro (1867); Ma: Colmeiro (1867), Herrera et al. (2007); Se: Barras (1896), Llimona \& Egea (1985), Rowe \& Egea (1985a, 1987a), Sáiz-Jiménez et al. (1991).

Lecanora muralis var. dubyi (Müll. Arg.) Poelt -Al: Egea \& Llimona (1981a, 1987); Ca: Rowe \& Egea (1987b), Rowe et al. (1996); Gr: Degelius (1966), Egea et al. (1982); Se: Rowe \& Egea (1985b, 1987a).

Lecanora muralis var. versicolor (Pers.) Tuck. - Al: Egea \& Llimona (1981a), Alonso \& Egea (1996); Gr: Colmeiro (1867), Sampaio (1917), Casares (1988, 1992), Casares \& Llimona $(1986,1989)$, Alonso \& Egea (1996).

Lecanora orosthea (Ach.) Ach. - Ca: Egea \& Rowe (1987), Rowe \& Egea (1986, 1988); Se: Rowe \& Egea (1987a). 
Lecanora peltata (Ramond) Steud. - Gr: Degelius (1966), Prieto \& Espinosa (1971).

Lecanora poeltiana Clauzade \& Cl. Roux - Gr: Casares \& Llimona (1989).

Lecanora polytropa (Ehrh.) Rabenh. - Al: Egea \& Llimona (1981a, 1987); Ca: Colmeiro (1867), Rowe et al. (1996); Gr: Casares \& Llimona (1982), Egea et al. (1982), Martínez \& Aragón (2004).

Lecanora prominens Asta et al. - Ca: Rowe et al. (1996); Gr: Casares \& Llimona $(1983,1989)$.

Lecanora pruinosa Chaub. - Se: Ariño et al. (1997).

Lecanora pulicaris (Pers.) Ach. - ${ }^{*}$ Co: MACB 103915; Ca: Fos (1998); Gr: Fos (1998); H: Fos (1998); Ma: Fos (1998).

Lecanora rubicunda Bagl. - Ca: Fos (1998).

Lecanora rubina (Vill.) Ach. - Gr: Prieto \& Espinosa (1971).

Lecanora rupicola (L.) Zahlbr. -Al: Colmeiro (1867), Egea \& Llimona (1981a, 1987); Ca: Colmeiro (1867), Rowe \& Egea (1987b), Rowe et al. (1996); Gr: Colmeiro (1867), Sampaio (1917), Prieto \& Espinosa (1971), Casares \& Llimona (1982), Egea et al. (1982).

Lecanora rupicola subsp. subplanata (Nyl.) Leuckert \& Poelt - Al: Egea \& Llimona (1994).

Lecanora saligna (Schrad.) Zahlbr. - Gr: Colmeiro (1867); J: Martínez \& Aragón (2004).

Lecanora schistina (Nyl.) Arnold - Al: Egea \& Llimona (1981a, b, 1983, 1987, 1994); Ca: Egea \& Rowe (1987); Ma: Herrera et al. (2007).

Lecanora sienae B. de Lesd. - Ca: Barreno et al. (1988).

Lecanora strobilina (Spreng.) Kieff. - Ca: Barreno et al. (1988), Fos (1998); H: Fos (1998); J: Aragón et al. (2006a); Ma: Seaward \& Arvidson (1997), Fos (1998).

Lecanora subcarnea (Lilj.) Ach. - Ca: Rowe \& Egea (1986, 1988); H: Rowe \& Egea (1987a); J: Sampaio (1917).

Lecanora subrugosa Nyl. - Ca: Barreno et al. (1988), Rowe et al. (1996), Fos (1998)

Lecanora sulphurata (Ach.) Nyl. - Al: Llimona (1975), Egea \& Llimona $(1983,1987,1994)$.

Lecanora sulphurea (Hoffm.) Ach. - Al: Egea \& Llimona (1981a, b, 1983, 1987, 1994); Ca: Rowe \& Egea (1987b), Rowe et al. (1996); Gr: Werner (1979), Casares \& Llimona (1989).

Lecanora sulphurella Hepp - Al: Llimona (1975), Egea \& Llimona $(1983,1994)$.

Lecanora swartzii (Ach.) Ach. - Al: Egea \& Llimona (1981a, 1987); Gr: Egea et al. (1982).

Lecanora sylvestris (Nyl.) Zahlbr. - Ca: Fos (1998).

Lecanora symmicta (Ach.) Ach. - Ca: Barreno et al. (1988), Fos (1998); J: Aragón et al. (2006a).

Lecanora umbrosa Degel. - Al: Egea \& Llimona (1981a); Gr: Egea et al. (1982).

Lecanora varia (Hoffm.) Ach. - Ca: Colmeiro (1867); J: Sarrión \& Burgaz (1995), Aragón et al. (2004c), Martínez \& Aragón (2004).

Lecidea atrobrunnea (Ramond) Schaer. -Al: Egea \& Llimona (1981a, 1987); Gr: Sampaio (1917), Degelius (1966), Casares \& Llimona (1982), Egea et al. (1982).

Lecidea auriculata Th. Fr. - Al: Egea \& Llimona (1981a, 1987); Gr: Degelius (1966).

Lecidea berengeriana (A. Massal.) Nyl. - Ca: Rowe et al. (1996).

Lecidea circinarioides Casares \& Hafellner-Al: Llimona (1974), Casares \& Gutiérrez (1993), Gutiérrez \& Casares (1994), Casares et al. (1996); Gr: Casares \& Gutiérrez (1993), Casares et al. (1996).

Lecidea conferenda Nyl. - Al: Egea \& Llimona (1981a, 1987); Gr: Egea et al. (1982), Pereira et al. (1987); Se: Rowe \& Egea (1985b).
Lecidea confluens (Weber) Ach. - Al: Colmeiro (1867), Egea \& Llimona (1981a, 1987); Ca: Colmeiro (1867); Gr: Colmeiro (1867), Casares \& Llimona (1982), Egea et al. (1982); J: Colmeiro (1867); Ma: Colmeiro (1867).

Lecidea exigua Chaub. - Ca: Rowe et al. (1996); J: Aragón et al. (2004c).

Lecidea fuscoatra (L.) Ach. -Al: Egea \& Llimona (1981a, 1987); Ca: Colmeiro (1867), Rowe \& Egea (1986, 1988), Rowe et al. (1996); Gr: Colmeiro (1867); H: Rowe \& Egea (1987a); Se: Rowe \& Egea (1985a, b, 1987a).

Lecidea grisella Flörke - Al: Egea \& Llimona (1994); Ca: Rowe \& Egea (1986, 1988); H: Rowe \& Egea (1987a); Se: Rowe \& Egea (1985a, b, 1987a).

Lecidea gypsicola Llimona-Al: Llimona (1974), Casares et al. (1996); Gr: Casares \& Gutiérrez (1993), Casares et al. (1996).

Lecidea holopolia (Tuck.) Zahlbr. - J: Aragón et al. (2006a).

Lecidea hypnorum Lib. - Ca: Rowe et al. (1996).

Lecidea hypopta Ach. - J: Aragón et al. (2006a).

Lecidea lactea Flörke ex Schaer. -Al: Egea \& Llimona (1981a, 1987).

Lecidea lithophila (Ach.) Ach. - Al: Egea \& Llimona (1981a, 1987); Gr: Sampaio (1917).

Lecidea mutabilis Clemente -Al: Colmeiro (1867); Ca: Colmeiro (1867).

Lecidea obluridata Nyl. - Al: Egea \& Llimona (1981a, 1987, 1994); Ca: Rowe \& Egea (1988); Se: Rowe \& Egea (1985b, 1987a).

Lecidea paratropoides Müll. Arg. - Ca: Rowe et al. (1996).

Lecidea promiscens Nyl. - Gr: Egea et al. (1982).

Lecidea promiscua Nyl. - Gr: Sampaio (1917).

Lecidea rhaetica Th. Fr. - Gr: Casares \& Llimona (1982).

Lecidea rimiseda Nyl. - Gr: Prieto \& Espinosa (1971), Egea et al. (1982).

Lecidea sanguineoatra (Wulfen) Ach. - Ca: Rowe et al. (1996).

Lecidea sarcogynoides Körb. - Al: Egea \& Llimona (1981a, 1987); Ca: Rowe \& Egea (1986, 1988); H: Rowe \& Egea (1987a); Se: Rowe \& Egea (1985a, b).

Lecidea subcinerascens Nyl. - Ca: Rowe et al. (1996).

Lecidea syncarpa Zahlbr. - Gr: Egea et al. (1982).

Lecidea tessellata Flörke - Al: Egea \& Llimona (1981a, 1987); Gr: Casares \& Llimona (1982), Egea et al. (1982).

Lecidella achristotera (Nyl.) Hertel \& Leuckert - Ca: Fos (1998); Gr: Fos (1998); H: Fos (1998); Ma: Fos (1998).

Lecidella alaiensis (Vain.) Hertel - Gr: Casares \& Llimona (1986, 1989).

Lecidella asema (Nyl.) Knoph \& Hertel-Al: Egea \& Llimona (1981a, b, 1983, 1987, 1994); Ca: Rowe \& Egea (1986).

Lecidella carpathica Körb. - Al: Egea \& Llimona (1981a, b), Llimona \& Egea (1985), Egea \& Llimona (1987); Ca: Rowe et al. (1996); *Co: MACB 106702; Gr: Werner (1979), Egea et al. (1982), Casares (1988), Casares \& Llimona (1986, 1989); H: Rowe \& Egea (1987a); Se: Rowe \& Egea (1987a).

Lecidella elaeochroma (Ach.) M. Choisy - Al: Colmeiro (1867), Llimona (1975); Ca: Colmeiro (1867), Rowe et al. (1996), Fos (1998); Gr: Colmeiro (1867); *Co: MACB 103336; H: Fos (1998); J: Aragón et al. (2004c, 2006a); Ma: Colmeiro (1867), Rowe et al. (1996).

Lecidella euphorea (Flörke) Hertel - Ca: Barreno et al. (1988), Fos (1998); H: Fos (1998); Ma: Martín-Osorio (1988).

Lecidella inamoena (Müll. Arg.) Hertel - Ca: Casares \& Rowe (1988), Rowe et al. (1996); Gr: Casares (1988), Casares \& Llimona (1986, 1989). 
Lecidella patavina (A. Massal.) Knoph \& Leuckert - Ma: Herrera et al. (2007).

Lecidella pulveracea (Schaer.) P. Syd. - J: Aragón et al. (2006a); Ma: Rowe et al. (1996).

Lecidella stigmatea (Ach.) Hertel \& Leuckert - Al: Egea \& Llimona (1981a, 1987), Alonso \& Egea (1996); Ca: Rowe \& Egea (1987b), Rowe et al. (1996); Gr: Degelius (1966), Werner (1979), Egea et al. (1982), Casares (1988), Casares \& Llimona (1989).

Lecidella stigmatea f. egena (Kremp.) Clauzade \& Cl. Roux - Gr: Egea et al. (1982).

Lempholemma elveloideum (Ach.) Zahlbr. - Gr: Casares \& Llimona (1989).

Lempholemma polyanthes (Bernh.) Malme - Gr: Egea et al. (1982).

Lepraria aeruginosa (F. H. Wigg.) Sm. - Ca: Rowe \& Egea (1987b), Barreno et al. (1988), Rowe et al. (1996), Hernández Gallego et al. (1998); H: Rowe et al. (1982), Rowe \& Egea (1987a).

Lepraria caesioalba (B. de Lesd.) J. R. Laundon - Ca: Fos (1998).

Lepraria incana (L.) Ach. - Al: Egea \& Llimona (1981a, 1994); Ca: Rowe \& Egea (1986, 1988), Egea \& Rowe (1987), Barreno et al. (1988), Fos (1998); Gr: Egea et al. (1982), Casares \& Llimona (1989); Ma: Rowe et al. (1996), Herrera et al. (2007); J: Aragón et al. (2004c, 2006a); Se: Rowe \& Egea (1985b, 1987a).

Lepraria isidiata (Llimona) Llimona \& A. Crespo - Al: Llimona (1974), Casares \& Gutiérrez (1993), Gutiérrez \& Casares (1994), Martínez-Sánchez et al. (1994), Guerra et al. (1995); Gr: Casares \& Gutiérrez (1993), Martínez-Sánchez et al. (1994).

Lepraria lobificans Nyl. - Ca: Rowe et al. (1996); J: Aragón et al. (2004c).

Lepraria neglecta (Nyl.) Erichsen - Ca: Rowe \& Egea (1987b), Rowe et al. (1996); Gr: Egea et al. (1982); Se: Rowe \& Egea (1985b, 1987a).

Lepraria nivalis J. R. Laundon - Al: Egea \& Llimona (1994), Alonso \& Egea (1996); Ca: Rowe \& Egea (1986), Barreno et al. (1988); Gr: Casares (1988), Casares \& Llimona (1986, 1989); H: Rowe et al. (1982); Ma: Seaward \& Arvidson (1997); Se: Rowe \& Egea (1985a, b), Ariño et al. (1997).

Lepraria vouauxii (Hue) R. C. Harris - Ca: Rowe et al. (1996); Ma: Seaward \& Arvidson (1997).

Leprocaulon quisquiliare (Leers) M. Choisy - Al: Casares \& Gutiérrez (1993), Egea \& Llimona (1994), Gutiérrez \& Casares (1994); Ca: Rowe \& Egea $(1986,1988)$, Egea \& Rowe (1987), Rowe et al. (1996); *Co: MACB 103102; Gr: Navás (1904), Casares \& Gutiérrez (1993); Ma: Rowe et al. (1996); Se: Rowe \& Egea (1985b, 1987a).

Leproloma membranaceum (Dicks.) Vain. - Ca: Rowe \& Egea (1986); Gr: Egea et al. (1982); Se: Rowe \& Egea (1985b).

Leproplaca chrysodeta (Vain. ex Räsänen) J. R. Laundon - Ca: Rowe et al. (1996).

Leproplaca xantholyta (Nyl.) Hue - Al: Casares \& Gutiérrez (1993), Gutiérrez \& Casares (1994), Alonso \& Egea (1996); Ca: Ariño et al. (1997), Rowe \& Egea (1988); Gr: Casares (1988), Casares \& Llimona (1989), Casares \& Gutiérrez (1993); Ma: Rowe et al. (1996); Se: Rowe \& Egea (1987a), Ariño et al. (1997).

Leptochidium albociliatum (Desm.) M. Choisy - Ca: Rowe \& Egea (1988); *Co: MACB 103110; Gr: Burgaz (2011a, b); H: Rowe \& Egea (1987a), Burgaz (2011a, b); J: Burgaz (2011a, b); Ma: Seaward \& Arvidson (1997), Burgaz (2011a, b).

Leptogium aragonii Otálora - J: Otálora et al. (2008).

Leptogium azureum (Sw. ex Ach.) Mont. - Ca: Aragón et al. (2004a).

Leptogium brebissonii Mont. - Ca: Rowe et al. (1996); J: Aragón \& Rico (1997); Ma: Seaward \& Arvidson (1997).
Leptogium corticola (Taylor) Tuck. - Ma: Rowe et al. (1996).

Leptogium cyanescens (Pers.) Körb. - Ca: Fos (1998); Ma: Seaward \& Arvidson (1997).

Leptogium ferax (Durieu \& Mont.) Rabenh. - Ma: Seaward \& Arvidson (1997).

Leptogium furfuraceum (Harm.) Sierk - Ma: Rowe et al. (1996), Seaward \& Arvidson (1997).

Leptogium gelatinosum (With.) J. R. Laundon-Ca: Rowe et al. (1996); *Co: MACB 103916; Gr: Otálora et al. (2008); Ma: Martín-Osorio (1988), Rowe et al. (1996).

Leptogium lichenoides (L.) Zahlbr. - Ca: Rowe et al. (1996); *Co: MACB 103339; Gr: Casares (1988), Casares \& Llimona (1989); J: Aragón \& Rico (1997), Aragón \& Sarrión (2003), Aragón et al. (2004c, 2006a), Otálora et al. (2004, 2008); Ma: Rowe et al. (1996), Otálora et al. (2008).

Leptogium massiliense Nyl. - Ca: Casares \& Rowe (1988); Gr: Casares \& Llimona (1989); J: Aragón \& Rico (1997).

Leptogium palmatum (Huds.) Mont. - Ca: Barreno et al. (1988), Rowe \& Egea (1988).

Leptogium pulvinatum (Hoffm.) Otálora - *Co: MACB 103344; Ma: Otálora et al. (2008).

Leptogium pulvinatum var. quercicola (Otálora et al.) Otálora - Al: Otálora et al. (2004); J: Otálora et al. (2008).

Leptogium saturninum (Dicks.) Nyl. - Ca: Rowe et al. (1996); J: Aragón \& Rico (1997); Ma: Rowe et al. (1996), Seaward \& Arvidson (1997).

Leptogium schraderi (Bernh.) Nyl. - J: Aragón \& Rico (1997); Ma: Seaward \& Arvidson (1997).

Leptogium subaridum P. M. Jørg. \& Goward - J: Aragón et al. (2004b).

Leptogium subtile (Schrad.) Torss. - Ca: Rowe et al. (1996); J: Aragón \& Sarrión (2003).

Leptogium teretiusculum (Flörke) Arnold - Ca: Rowe et al. (1996), Fos (1998); J: Aragón \& Sarrión (2003); Ma: Fos (1998).

Leptorhaphis oleae (A. Massal.) Körb. - Ca: Rowe et al. (1996).

\#Leptosphaeria ramalinae (Desm.) Sacc. - Ma: Rowe et al. (1996).

Lethariella intricata (Moris) Krog - Ca: Rowe \& Egea (1987b), Rowe et al. (1996).

\#Lichenochora mediterraneae Calat. et al. - Ca: Calatayud et al. (2000).

\#Lichenodiplis lecanorae (Vouaux) Dyko \& D. Hawksw. - Ma: Rowe et al. (1996).

Lichenomphalia meridionalis (Contu \& La Rocca) P. A. Moreau \& Courtec. - *H: MACB 103346; J: Rico \& Barrasa (2011).

Lichenomphalia umbellifera (L.: Fr.) Redhead et al. - Gr: Rico \& Barrasa (2011).

Lichenomphalia velutina (Quél.) Redhead et al. - H: Rico \& Barrasa (2011).

\#Lichenopuccinia poeltii D. Hawksw. \& Hafellner - Ma: Rowe et al. (1996).

\#Lichenostigma diploiciae Calat. et al. - Al: Calatayud et al. (2002).

Lichina pygmaea (Lightf.) C. Agardh - Ca: Colmeiro (1867).

Lichinella algerica (J. Steiner) P. P. Moreno \& Egea - Al: Moreno \& Egea (1989), Egea \& Llimona (1994).

Lichinella cribellifera (Nyl.) P. P. Moreno \& Egea - Al: Egea \& Llimona (1981a, 1983, 1985, 1987, 1994); Ma: Herrera et al. (2007).

Lichinella sinaica (Galun \& Marton) P. P. Moreno \& Egea -Al: Moreno \& Egea (1989).

Lichinella stipatula Nyl. - Al: Egea \& Llimona (1981a, 1983, 1987, 1994) Llimona \& Egea (1985); Ca: Rowe \& Egea (1988); H: Rowe \& Egea (1987a); Ma: Herrera et al. (2007); Se: Llimona \& Egea (1985), Rowe \& Egea (1985a, b). 
Lithographa graphidioides (Cromb.) Imshaug ex Coppins \& Fryday Ma: Rowe et al. (1996).

\#Llimoniella scabridula (Müll. Arg.) Nav.-Ros. \& Hafellner-Al: Casares \& Gutiérrez (1993), Guerra et al. (1995).

Lobaria amplissima (Scop.) Forssell - Ca: Aragón \& Martínez (1995), Fos (1998), Hernández Gallego et al. (1998); J: Aragón et al. (2006a); Ma: Colmeiro (1867), Martín-Osorio (1988), Rowe et al. (1996), Fos (1998), Burgaz \& Martínez (2003), Seaward \& Arvidson (1997).

Lobaria pulmonaria (L.) Hoffm. -Al: Colmeiro (1867); Ca: Colmeiro (1867), Aragón \& Martínez (1995), Rowe et al. (1996), Hernández Gallego et al. (1998); H: Rowe et al. (1982), Aragón \& Martínez (1995); J: Aragón \& Rico (1997), Burgaz \& Martínez (2003), Aragón et al. (2006a); Ma: Colmeiro (1867), Martín-Osorio (1988), Rowe et al. (1996), Seaward \& Arvidson (1997), Burgaz \& Martínez (2003).

Lobaria scrobiculata (Scop.) P. Gaertn. - Ca: Colmeiro (1867), Aragón \& Martínez (1995), Rowe et al. (1996), Fos (1998), H: Burgaz \& Martínez (2003); J: Aragón \& Rico (1997), Aragón et al. (2006a); Ma: Martín-Osorio (1988), Rowe et al. (1996), Seaward \& Arvidson (1997), Fos (1998), Burgaz \& Martínez (2003).

**Lobaria virens (With.) J. R. Laundon - Ca: MACB 103746.

Lobothallia alphoplaca (Wahlenb.) Hafellner - Gr: Colmeiro (1867), Egea et al. (1982).

Lobothallia farinosa (Hoffm) A. Nordin et al. - Al: Alonso \& Egea (1996); Ca: Alonso \& Egea (1996); Gr: Casares \& Llimona (1986, 1989).

Lobothallia radiosa (Hoffm) Hafellner -Al: Llimona (1974), Egea \& Llimona (1981a, 1983, 1987, 1994, 1997), Alonso \& Egea (1996); Ca: Casares \& Rowe (1988), Rowe \& Egea (1986, 1988), Rowe et al. (1996); *Co: MACB 103730; Gr: Colmeiro (1867), Sampaio (1917), Casares \& Llimona (1986, 1989), Alonso \& Egea (1996), Egea et al. (1982); H: Rowe \& Egea (1987a); Se: Barras (1896), Rowe \& Egea (1987a), Rowe \& Sainz-Jiménez (1988).

Lopadium disciforme (Flot.) Kullh. - Ma: Rowe et al. (1996).

Macentina dictyospora Orange - J: Aragón \& Sarrión (2003).

**Marchandiomyces corallinus (Roberge) Diederich \& D. Hawksw. *Co: MACB 103347.

Maronea constans (Nyl.) Hepp - Ca: Rowe et al. (1996); Ma: Rowe et al. (1996).

**Massalongia carnosa (Dicks.) Körb. - Co: MACB 103111.

Megalospora tuberculosa (Fée) Sipman - Gr: Colmeiro (1867).

Megaspora verrucosa (Ach.) Hafellner \& V. Wirth - Ca: Colmeiro (1867), Rowe et al. (1996); Gr: Werner (1979), Casares (1986, 1988), Casares \& Llimona (1983, 1989); J: Aragón et al. (2004c, 2006a); Ma: Rowe et al. (1996).

Melanelixia fuliginosa (Fr. ex Duby) O. Blanco et al. subsp. fuliginosa - Ca: Rowe \& Egea (1986, 1988), Egea \& Rowe (1987), Hernández Gallego et al. (1998), Fos (1998); J: Aragón \& Rico (1997), Aragón et al. (2004c, 2006a).

Melanelixia glabra (Schaer.) O. Blanco et al. - Ca: Barreno et al. (1988), Fos (1998); *Co: MACB 103349; Gr: Fos (1998); J: Aragón \& Rico (1997), Aragón et al. (2006a); Ma: Rowe et al. (1996).

Melanelixia glabratula (Lamy) Sandler \& Arup - Ca: Rowe \& Egea (1986, 1988), Barreno et al. (1988), Rowe et al. (1996), Fos (1998); *Ma: MACB 106381; Se: Rowe \& Egea (1987a).

Melanelixia subaurifera (Nyl.) O. Blanco et al. - Ca: Barreno et al. (1988), Rowe et al. (1996); Gr: Fos (1998); H: Fos (1998); Ma: Rowe et al. (1996), Fos (1998).
Melanohalea elegantula (Zahlbr.) 0. Blanco et al. - J: Aragón et al. (2006a); Ma: Martín-Osorio (1988).

Melanohalea exasperata (De Not.) O. Blanco et al. - Ca: Colmeiro (1867), Barreno et al. (1988), Rowe et al. (1996), Fos (1998); *Co: MACB 103353; Gr: Fos (1998); J: Aragón et al. (2006a); Ma: Martín-Osorio (1988), Rowe et al. (1996), Fos (1998).

Melanohalea exasperatula (Nyl.) O. Blanco et al. - Gr: Casares \& Llimona $(1986,1989)$.

Melanohalea infumata (Nyl.) O. Blanco et al. - Al: Egea \& Llimona (1981a, 1983, 1987); Gr: Casares \& Llimona (1982), Egea et al. (1982).

Melanohalea laciniatula (Flagey ex H.Olivier) 0. Blanco et al. - J: Aragón \& Rico (1997), Aragón et al. (2004c, 2006a); Ma: Martín-Osorio (1988), Rowe et al. (1996).

Micarea denigrata (Fr.) Hedl. - J: Aragón et al. (2006a).

Micarea peliocarpa (Anzi) Coppins - Ca: Rowe \& Egea (1986), Rowe et al. (1996); Ma: Rowe et al. (1996), Martínez \& Hafellner (1998); Se: Rowe \& Egea (1987a).

Micarea prasina Fr. - J: Aragón et al. (2006a).

Microcalicium disseminatum (Ach.) Vain. - J: Aragón et al. (2006a).

Miriquidica deusta (Stenh.) Hertel \& Rambold - Ca: Rowe \& Egea (1986, 1988), Rowe et al. (1996); H: Rowe \& Egea (1987a); Se: Rowe \& Egea (1985a, 1987a).

Miriquidica garovaglioi (Schaer.) Hertel \& Rambold - Gr: Colmeiro (1867).

Miriquidica griseoatra (Hoffm.) Hertel \& Rambold-Al: Egea \& Llimona (1981a); Ca: Rowe \& Egea (1986, 1987b), Egea \& Llimona (1987), Rowe et al. (1996); Gr: Egea et al. (1982); H: Rowe \& Egea (1987a); Se: Rowe \& Egea (1985b).

Moelleropsis nebulosa (Hoffm.) Gyeln. - *Co: MACB 103352; Ma: Seaward \& Arvidson (1997)

Montanelia disjuncta (Erichsen) Divakar et al. -Al: Egea \& Llimona (1987).

Montanelia sorediata (Ach.) Divakar et al. - Gr: Casares \& Llimona (1982); Se: Silvestre \& Rowe (1982).

\#Muellerella lichenicola (Sommerf.) D. Hawksw. - Ca: Rowe et al. (1996).

Mycocalicium subtile (Pers.) Szatala - Ca: Colmeiro (1867); Sarrión et al. (1999), Muñiz \& Hladun (2011); Gr: Colmeiro (1867); Fos (1998); J: Sarrión et al. (1999), Aragón et al. (2004c, 2006a), Muñiz \& Hladun (2011); Ma: Sarrión et al. (1999).

Mycocalicium victoriae (C. Knight ex F. Wilson) Nádv. - Gr: Muñiz \& Hladun (2007), Muñiz \& Hladun (2011).

Mycomicrothelia confusa D. Hawksw. - Ca: Rowe et al. (1996).

Mycoporum antecellens (Nyl.) R. C. Harris - Ca: Fos (1998).

\#Nectriopsis lecanodes (Ces.) Diederich \& Schroers - Ma: Rowe et al. (1996).

Neocatapyrenium cladonioideum (Vain.) H. Harada - J: Prieto et al. (2010).

Nephroma laevigatum Ach. - Ca: Colmeiro (1867), Abbayes (1945), Martínez \& Aragón (1994), Barreno et al. (1988), Rowe et al. (1996), Fos (1998), Hernández Gallego et al. (1998), Rowe \& Egea (1988); H: Rowe et al. (1982); Martínez \& Aragón (1994), Burgaz \& Martínez (2003); J: Sampaio (1917), Aragón \& Rico (1997), Burgaz \& Martínez (2003), Aragón et al. (2006a); Ma: Colmeiro (1867), Martín-Osorio (1988), Martínez \& Aragón (1994); Rowe et al. (1996), Fos (1998), Burgaz \& Martínez (2003).

Nephroma resupinatum (L.) Ach. - Ma: Martínez \& Aragón (1994).

Nephroma tangeriense (Maheu \& A. Gillet) Zahlbr. - Ca: Rowe et al. (1996), Fos (1998); H: Burgaz \& Martínez (2003); Ma: Rowe et 
al. (1996), Seaward \& Arvidson (1997), Burgaz \& Martínez (2003).

Normandina pulchella (Borrer) Nyl. - Ca: Rowe et al. (1996), Fos (1998); Ma: Rowe et al. (1996), Fos (1998).

\#Norrlinia peltigericola (Nyl.) Theiss. \& Syd. - J: Martínez \& Hafellner (1998), Martínez (1999).

Obryzum corniculatum (Hoffm.) Wallr. - Ca: Fos (1998); *Co: MACB 103101; Gr: Colmeiro (1867); Ma: Seaward \& Arvidson (1997).

Ochrolechia alboflavescens (Wulfen) Zahlbr. - Ca: Fos (1998); J: Aragón et al. (2006a); Ma: Rowe et al. (1996).

Ochrolechia balcanica Verseghy - Ca: Fos (1998); Ma: Seaward \& Arvidson (1997), Kukwa (2011).

Ochrolechia crozalsiana Clauzade \& Vězda - Ma: Kukwa (2011).

Ochrolechia dalmatica (Erichsen) Boqueras - J: Aragón et al. (2006a); Ma: Boqueras (1997).

Ochrolechia pallescens (L.) A. Massal. - Ca: Barreno et al. (1988), Fos (1998); J: Aragón et al. (2006a); Ma: Boqueras (1997), Fos (1998).

Ochrolechia parella (L.) A. Massal. - Al: Egea \& Llimona (1981a, b, 1983, 1987, 1994), Kukwa (2011); Ca: Colmeiro (1867), Sampaio (1917), Rowe \& Egea (1986, 1988), Egea \& Rowe (1987), Rowe et al. (1996), Fos (1998); Gr: Colmeiro (1867); H: Rowe \& Egea (1987a); Ma: Colmeiro (1867), Rowe et al. (1996); Se: Rowe \& Egea (1985b).

Ochrolechia subviridis (Høeg) Erichsen - Ca: Rowe et al. (1996), Fos (1998); Ma: Rowe et al. (1996).

Ochrolechia szatalaensis Verseghy - J: Aragón et al. (2006a).

Ochrolechia tartarea (L.) Zahlbr. - Ca: Colmeiro (1867), Silvestre \& Rowe (1982); Ma: Rowe et al. (1996).

Ochrolechia turneri (Sm.) Hasselrot - Ca: Fos (1998); J: Aragón et al. (2006a); Ma: Seaward \& Arvidson (1997), Kukwa (2011).

Opegrapha celtidicola Jatta - H: Torrente \& Egea (1989a), Torrente \& Egea (1989b).

Opegrapha corticola Coppins \& P. James - Ca: Rowe et al. (1996).

Opegrapha diaphoroides Nyl. - Ca: Barreno et al. (1988).

Opegrapha herbarum Mont. - Al: Torrente \& Egea (1989a); Ca: Fos (1998); Ma: Martín-Osorio (1988).

Opegrapha lutulenta Nyl. -Al: Torrente \& Egea (1989a), Egea \& Llimona (1994); Ca: Rowe \& Egea (1986), Egea \& Rowe (1987), Torrente \& Egea (1989a); Ma: Herrera et al. (2007).

Opegrapha multipuncta Coppins \& P. James - Ca: Rowe et al. (1996).

\#Opegrapha rupestris Pers. -Al: Egea \& Torrente (1985), Torrente \& Egea (1989a); Ca: Colmeiro (1867).

Opegrapha saxigena Taylor - Ca: Torrente \& Egea (1989a).

Opegrapha vulgata (Ach.) Ach. - Al: Colmeiro (1867); Ca: Colmeiro (1867), Fos (1998); Gr: Colmeiro (1867); Ma: Martín-Osorio (1988).

Pachyphiale carneola (Ach.) Arnold - Ca: Rowe et al. (1996).

Pannaria conoplea (Ach.) Bory - J: Aragón \& Rico (1997), Carballal et al. (2010a); Ma: Seaward \& Arvidson (1997).

Pannaria rubiginosa (Thunb.) Delise - Ca: Rowe et al. (1996); J: Aragón \& Rico (1997), Carballal et al. (2010a); Ma: Seaward \& Arvidson (1997), Carballal et al. (2010a).

Parabagliettoa cyanea (A. Massal.) Gueidan \& Cl. Roux - Al: Alonso \& Egea (1996); Gr: Alonso \& Egea (1996).

Parabagliettoa dufourii (DC.) Gueidan \& Cl. Roux - Gr: Casares \& Llimona (1984).

Paralecanographa grumulosa (Dufour) Ertz \& Tehler - Al: Llimona \& Werner (1975), Egea \& Llimona (1983, 1984, 1994). Ca: Rowe \& Egea (1986), Torrente \& Egea (1989a); Gr: Casares \& Llimona (1989); Ma: Herrera et al. (2007).
Parmelia omphalodes (L.) Ach. - Ca: Colmeiro (1867), Rowe \& Egea (1987b), Rowe et al. (1996); Ma: Colmeiro (1867).

Parmelia saxatilis (L.) Ach. - Ca: Colmeiro (1867), Rowe \& Egea (1986, 1988), Egea \& Rowe (1987), Barreno et al. (1988), Fos (1998); Gr: Colmeiro (1867); H: Rowe et al. (1982), Fos (1998); J: Aragón \& Rico (1997), Aragón et al. (2004c, 2006a); Ma: Colmeiro (1867), Martín-Osorio (1988), Rowe et al. (1996), Seaward \& Arvidson (1997), Fos (1998); Se: Rowe \& Egea (1985a, b, 1987a).

Parmelia submontana Hale - Ca: Rowe et al. (1996); J: Aragón \& Rico (1997), Aragón et al. (2004c, 2006a), Molina et al. (2004); Ma: Martín-Osorio (1988), Rowe et al. (1996).

Parmelia sulcata Taylor -Al: Egea \& Llimona (1981a, 1987); Ca: Barreno et al. (1988), Fos (1998), Hernández Gallego et al. (1998); H: Rowe et al. (1982), Fos (1998); J: Navás (1901), Aragón \& Rico (1997), Aragón et al. (2006a); Ma: Abbayes (1945), MartínOsorio (1988), Rowe et al. (1996), Fos (1998).

Parmeliella parvula P. M. Jørg. - Ma: López de Silanes et al. (2010).

Parmeliella testacea P. M. Jørg. - J: Aragón \& Rico (1997); Ma: Rowe et al. (1996), Seaward \& Arvidson (1997).

Parmeliella triptophylla (Ach.) Müll. Arg. - Ca: Colmeiro (1867), Sequeiros et al. (1986), Barreno et al. (1988); J: Aragón \& Rico (1997); Ma: Colmeiro (1867).

Parmelina carporrhizans (Taylor) Poelt \& Vězda - *Co: MACB 103418; Ma: Martín-Osorio (1988), Rowe et al. (1996).

Parmelina pastillifera (Harm.) Hale - Ma: Rowe et al. (1996).

Parmelina quercina (Willd.) Hale - Ca: Rowe \& Egea (1987b), Barreno et al. (1988), Rowe et al. (1996), Fos (1998), Hernández Gallego et al. (1998); *Co: MACB 103419; Gr: Fos (1998), Werner (1979); J: Aragón \& Rico (1997); Ma: Rowe et al. (1996), Seaward \& Arvidson (1997), Fos (1998); Se: Silvestre \& Rowe (1982).

Parmelina tiliacea (Hoffm.) Hale -Al: Egea \& Llimona (1981a, 1983, 1987, 1994); Ca: Colmeiro (1867), Rowe \& Egea (1986, 1988), Barreno et al. (1988), Rowe et al. (1996), Fos (1998), Hernández Gallego et al. (1998); *Co: MACB 103421; Gr: Colmeiro (1867), Casares \& Llimona (1982); H: Rowe et al. (1982), Fos (1998); J: Sarrión \& Burgaz (1995), Aragón \& Rico (1997), Aragón et al. (2006a); Ma: Colmeiro (1867), Martín-Osorio (1988), Rowe et al. (1996), Fos (1998); Se: Barras (1896, 1899), Rowe \& Egea (1985b, 1987a), Fos (1998).

Parmelinopsis minarum (Vain.) Elix \& Hale - Ca: Fos (1998).

Parmeliopsis ambigua (Wulfen) Nyl. - J: Aragón \& Rico (1997), Aragón et al. (2006a).

Parmotrema austrosinense (Zahlbr.) Hale - Ca: Barreno et al. (1988).

Parmotrema hypoleucinum (J. Steiner) Hale - Ca: Barreno et al. (1988), Fos (1998); H: Fos (1998); Ma: Seaward \& Arvidson (1997).

Parmotrema perlatum (Huds.) M. Choisy - Ca: Colmeiro (1867), Rowe \& Egea (1986), Barreno et al. (1988), Fos (1998), Hernández Gallego et al. (1998); Gr: Colmeiro (1867); H: Fos (1998); Ma: Colmeiro (1867), Martín-Osorio (1988), Fos (1998); Se: González-Fragoso (1883), Barras (1896), Navás (1901), Silvestre \& Rowe (1982).

Parmotrema pseudoreticulatum (Tav.) Hale - Ca: Divakar et al. (2005); Ma: Martín-Osorio (1988), Seaward \& Arvidson (1997).

Parmotrema reticulatum (Taylor) M. Choisy - Ca: Sampaio (1917), Barreno et al. (1988), Rowe \& Egea (1988), Rowe et al. (1996), Fos (1998); H: Fos (1998), Ma: Fos (1998); Ma: Divakar et al. (2005).

Parmotrema robustum (Degel.) Hale - Ca: Fos (1998). 
Parmotrema stuppeum (Taylor) Hale - Ca: Barreno et al. (1988), Rowe et al. (1996), Fos (1998); Ma: Fos (1998).

Parmotrema tinctorum (Despr. ex Nyl.) Hale - Ca: Rowe et al. (1996); Ma: Rowe et al. (1996).

Parmularia demissa (Flot.) Groz - Ca: Rowe \& Egea (1988); Se: Rowe \& Egea (1985a, 1987a).

Peccania cerebriformis Henssen \& Bedel -Al: Moreno \& Egea (1992).

Peccania coralloides (A. Massal.) A. Massal. - Al: Egea \& Llimona (1994); Gr: Casares \& Llimona (1989), Moreno \& Egea (1992); Ma: Seaward \& Arvidson (1997).

Peccania font-queriana P. P. Moreno \& Egea - Al: Moreno \& Egea (1992).

Peltigera canina (L.) Willd. - Al: Martínez (1999), Burgaz \& Martínez (2003); Ca: Martínez (1999); Gr: Colmeiro (1867), Martínez (1999), Burgaz \& Martínez (2003); H: Rowe et al. (1982), Martínez (1999); J: Colmeiro (1867), Martínez (1999); Ma: Colmeiro (1867), Martín-Osorio (1988), Martínez (1999), Burgaz \& Martínez (2003); Se: Lázaro Ibiza (1898b).

Peltigera collina (Ach.) Röhl. - Ca: Martínez \& Aragón (1994), Martínez (1999); Gr: Casares \& Llimona (1989), Martínez \& Aragón (1994), Martínez (1999), Burgaz \& Martínez (2003); H: Martínez (1999), Burgaz \& Martínez (2003); J: Martínez \& Aragón (1994), Aragón \& Rico (1997), Martínez (1999), Aragón et al. (2006a); Ma: Martín-Osorio (1988), Martínez \& Aragón (1994), Rowe et al. (1996), Seaward \& Arvidson (1997), Martínez (1999), Burgaz \& Martínez (2003).

Peltigera didactyla (With.) J. R. Laundon - Ma: Seaward \& Arvidson (1997).

**Peltigera elisabethae Gyeln. - Gr: MACB 103868.

Peltigera horizontalis (Huds.) Baumg. - J: Martínez (1999), Burgaz \& Martínez (2003); Se: Silvestre \& Rowe (1982).

Peltigera leucophlebia (Nyl.) Gyeln. - Ca: Rowe et al. (1996); Gr: Casares \& Llimona (1989).

Peltigera membranacea (Ach.) Nyl. - *H: 103765; Ma: Martínez (1999), Burgaz \& Martínez (2003).

Peltigera monticola Vitik. - *Gr: MACB 103767; J: Aragón \& Rico (1997), Martínez (1999), Burgaz \& Martínez (2003); Ma: Martínez (1999), Burgaz \& Martínez (2003).

Peltigera neckeri Hepp ex Müll. Arg. - Al: Martínez (1999), Burgaz \& Martínez (2003); Ca: Martínez (1999); Gr: Martínez (1999), Burgaz \& Martínez (2003); J: Martínez (1999), Burgaz \& Martínez (2003); Ma: Martínez (1999), Burgaz \& Martínez (2003).

Peltigera polydactylon (Neck.) Hoffm. - Ca: Rowe \& Egea (1986), Martínez (1999); Gr: Martínez (1999), Burgaz \& Martínez (2003); J: Martínez (1999), Burgaz \& Martínez (2003).

Peltigera ponojensis Gyeln. - Al: Martínez (1999), Burgaz \& Martínez (2003); Gr: Martínez (1999), Burgaz \& Martínez (2003); J: Aragón \& Rico (1997), Martínez (1999), Burgaz \& Martínez (2003); Ma: Martínez (1999), Burgaz \& Martínez (2003).

Peltigera praetextata (Flörke ex Sommerf.) Vain. - Ca: Barreno et al. (1988), Martínez (1999), Gr: Casares (1988), Martínez (1999), Burgaz \& Martínez (2003); J: Aragón \& Rico (1997), Martínez (1999), Burgaz \& Martínez (2003); Ma: Martínez (1999), Burgaz \& Martínez (2003).

Peltigera rufescens (Weiss) Humb. - Al: Martínez (1999), Burgaz \& Martínez (2003); Ca: Colmeiro (1867), Rowe et al. (1996); Gr: Sampaio (1917), Werner (1979), Casares (1988), Martínez (1999), Burgaz \& Martínez (2003); J: Aragón \& Rico (1997), Martínez (1999), Burgaz \& Martínez (2003); H: Rowe et al. (1982); Ma: Rowe et al. (1996), Martínez (1999), Burgaz \& Martínez (2003).
Peltigera venosa (L.) Hoffm. - Ca: Abbayes (1945).

Peltula crispatula (Nyl.) Egea - Al: Egea (1989).

Peltula euploca (Ach.) Poelt ex Ozenda \& Clauzade -Al: Rowe \& Egea (1986, 1988), Barreno et al. (1988); Ca: Rowe \& Egea (1986, 1988), Egea (1989); Ma: Colmeiro (1867), Herrera et al. (2007); Se: Llimona \& Egea (1985), Rowe \& Egea (1985a, b, 1987a), Egea (1989).

Peltula obscurans (Nyl.) Gyeln. -Al: Egea \& Llimona (1981b, 1983, 1994), Llimona \& Egea (1985), Egea (1989), Alonso \& Egea (1996); Ma: Alonso \& Egea (1996), Herrera et al. (2007).

Peltula obscurans var. hassei (Zahlbr.) Wetmore -Al: Egea \& Llimona (1981a, 1983).

Peltula omphaliza (Nyl.) Wetmore-Al: Egea \& Llimona (1981b, 1983, 1994), Llimona \& Egea (1985), Egea (1989); Se: Llimona \& Egea (1985), Rowe \& Egea (1985a, b), Egea (1989).

Peltula patellata (Bagl.) Swinscow \& Krog - Al: Egea (1989), Casares \& Gutiérrez (1993), Gutiérrez \& Casares (1994), Alonso \& Egea (1996).

Peltula placodizans (Zahlbr.) Wetmore -Al: Egea \& Llimona (1981a, 1983, 1985, 1994), Egea (1989).

Peltula zahlbruckneri (Hasse) Wetmore - Se: Rowe \& Egea (1985a, b).

Pertusaria albescens (Huds.) M. Choisy \& Werner - Al: Colmeiro (1867), Egea \& Llimona (1994); Ca: Colmeiro (1867), Sampaio (1917), Barreno et al. (1988), Rowe et al. (1996), Fos (1998), Hernández Gallego et al. (1998); * Co: MACB 103161; Gr: Colmeiro (1867), Casares (1988), Casares \& Llimona (1989); H: Fos (1998); J: Aragón et al. (2004c, 2006a); Ma: Martín-Osorio (1988), Rowe et al. (1996), Boqueras (1997), Fos (1998).

Pertusaria amara (Ach.) Nyl. -Al: Egea \& Llimona (1981a); Ca: Rowe \& Egea (1986), Barreno et al. (1988), Fos (1998); *Co: MACB 103431; H: Rowe et al. (1982), Fos (1998), Hernández Gallego et al. (1998); J: Aragón et al. (2004c, 2006a); Ma: Martín-Osorio (1988), Rowe et al. (1996), Fos (1998); Se: Rowe \& Egea (1985b).

Pertusaria amarescens Nyl. - Ca: Barreno et al. (1988).

Pertusaria bryontha (Ach.) Nyl. - Ca: Barreno et al. (1988).

Pertusaria caesioalba (Flot.) Nyl. - Ca: Colmeiro (1867), Barreno et al. (1988); Ma: Martín-Osorio (1988).

Pertusaria chiodectonoides Bagl. ex A. Massal. - Gr: Prieto \& Espinosa (1971); Ma: Boqueras (1997).

Pertusaria coccodes (Ach.) Nyl. - Ca: Barreno et al. (1988), Fos (1998); *Co: MACB 103432; J: Aragón et al. (2006a); Ma: Martín-Osorio (1988), Rowe et al. (1996).

Pertusaria coronata (Ach.) Th. Fr. - J: Aragón et al. (2004c, 2006a).

Pertusaria dalmatica Erichsen - Ca: Barreno et al. (1988).

Pertusaria dealbescens Erichsen - Ca: Boqueras (1997).

Pertusaria dispar J. Steiner - Ca: Fos (1998).

Pertusaria excludens Nyl. - Ca: Fos (1998).

Pertusaria ficorum Zahlbr. - Ca: Barreno et al. (1988), Fos (1998).

Pertusaria flavicans Lamy-Al: Egea \& Llimona (1981a, 1983, 1987); Se: Rowe \& Egea (1985b).

Pertusaria flavida (DC.) J. R. Laundon - Ca: Colmeiro (1867), Sampaio (1917), Barreno et al. (1988), Rowe et al. (1996), Fos (1998), Hernández Gallego et al. (1998); *Co: MACB 103433; H: Fos (1998); J: Sarrión \& Burgaz (1995), Aragón et al. (2006a); Ma: Martín-Osorio (1988), Rowe et al. (1996), Fos (1998).

Pertusaria hemisphaerica (Flörke) Erichsen - Ca: Fos (1998); *Co: MACB 103435; H: Fos (1998); J: Aragón et al. (2004c, 2006a); Ma: Martín-Osorio (1988), Rowe et al. (1996), Fos (1998). 
Pertusaria heterochroa (Müll. Arg.) Erichsen - Ca: Barreno et al. (1988), Fos (1998).

Pertusaria hymenea (Ach.) Schaer. - Ca: Colmeiro (1867), Rowe \& Egea (1987b), Barreno et al. (1988), Fos (1998); Co: Colmeiro (1867); Gr: Colmeiro (1867).

Pertusaria lactea (L.) Arnold -Al: Egea \& Llimona (1983); Ca: Egea \& Rowe (1987).

Pertusaria lactea f. faginea Erichsen - Al: Egea \& Llimona (1981a); Ca: Rowe \& Egea (1986), Fos (1998).

Pertusaria lecanorodes Erichsen - Ca: Barreno et al. (1988).

Pertusaria leioplaca DC. - Ca: Barreno et al. (1988), Fos (1998); *Co: MACB 103436.

Pertusaria leucosora Nyl. - Al: Egea \& Llimona (1981a, b, 1983, 1987); Ca: Rowe \& Egea (1986, 1988), Egea \& Rowe (1987), Rowe et al. (1996); H: Rowe \& Egea (1987a); Se: Rowe \& Egea (1985a, b, 1987a).

Pertusaria mammosa Harm. - Ca: Rowe \& Egea (1986), Egea \& Rowe (1987).

Pertusaria monogona Nyl. - Ca: Rowe \& Egea (1986), Egea \& Rowe (1987); Ma: Seaward \& Arvidson (1997).

Pertusaria multipuncta (Turner) Nyl. - Al: Egea \& Llimona (1984); Ca: Fos (1998); Ma: Martín-Osorio (1988).

Pertusaria ophthalmiza (Nyl.) Nyl. - Ca: Fos (1998); J: Aragón et al. (2006a).

Pertusaria paramerae A. Crespo \& Vězda - J: Aragón et al. (2004c, 2006a); Ma: Rowe et al. (1996).

Pertusaria pertusa (L.) Tuck. - Al: Colmeiro (1867), Egea \& Llimona (1981a, 1983, 1987); Ca: Colmeiro (1867), Rowe \& Egea (1986), Egea \& Rowe (1987), Barreno et al. (1988), Rowe et al. (1996); *Co: MACB 103437; Gr: Colmeiro (1867); H: Fos (1998); Ma: Martín-Osorio (1988), Rowe et al. (1996), Seaward \& Arvidson (1997), Fos (1998).

Pertusaria pluripuncta Nyl. - Al: Llimona (1975), Egea \& Llimona (1981b, 1983, 1987, 1994); Ca: Rowe \& Egea (1986), Egea \& Rowe (1987), Rowe et al. (1996); Ma: Herrera et al. (2007).

Pertusaria pseudocorallina (Lilj.) Arnold - Al: Egea \& Llimona (1981a, b, 1983, 1987); Ca: Rowe \& Egea (1986, 1988), Egea \& Rowe (1987), Barreno et al. (1988), Rowe et al. (1996), Fos (1998).

Pertusaria pustulata (Ach.) Duby - Ca: Colmeiro (1867), Barreno et al. (1988), Rowe et al. (1996), Fos (1998).

Pertusaria rupicola (Fr.) Harm. - Ca: Egea \& Rowe (1987), Rowe \& Egea (1987b), Rowe et al. (1996).

Pertusaria teneriffensis Vain. -Al: Egea \& Llimona (1983, 1994).

Pertusaria velata (Turner) Nyl. - Ca: Barreno et al. (1988), Fos (1998).

Pertusaria werneriana Boqueras - Ca: Boqueras (1997).

Peterjamesia circumscripta (Taylor) D. Hawksw. -Al: Torrente \& Egea (1989a), Egea \& Llimona (1994).

\#Pezizella epithallina (W. Phillips \& Plowr.) Sacc. - Ma: Martínez \& Hafellner (1998), Martínez (1999).

\#Phacographa glaucomaria (Nyl.) Hafellner - Al: Hafellner \& Sancho (1990).

Phacopsis oxyspora (Tul.) Triebel \& Rambold -Al: Hafellner \& Sancho (1990).

Phaeographis lyellii (Sm.) Zahlbr. - Ca: Sequeiros et al. (1986), Barreno et al. (1988).

Phaeophyscia endococcina (Körb.) Moberg - Al: Egea \& Llimona (1981a), Pedreño et al. (1987); Gr: Egea et al. (1982), Pereira et al. (1987).

Phaeophyscia hirsuta (Mereschk.) Essl. - Al: Alonso \& Egea (1996); Gr: Casares \& Llimona (1989).
**Pheophyscia hispidula (Ach.) Essl. - Co: MACB 106502.

Phaeophyscia insignis (Mereschk.) Moberg-Al: Pedreño et al. (1987).

Phaeophyscia nigricans (Flörke) Moberg - Ca: Casares \& Rowe (1988).

Phaeophyscia orbicularis (Neck.) Moberg - Al: Egea \& Llimona (1987), Pedreño et al. (1987); Ca: Casares \& Rowe (1988), Rowe et al. (1996), Fos (1998); *Co: MACB 103371; Gr: Casares (1988), Casares \& Llimona (1986, 1989), Fos (1998); J: Pedreño et al. (1987), Aragón \& Rico (1997), Aragón et al. (2006a); Ma: Martín-Osorio (1988); Se: Rowe \& Egea (1987a).

Phaeophyscia sciastra (Ach.) Moberg - Al: Pedreño et al. (1987).

Phlyctis agelaea (Ach.) Flot. - Ca: Barreno et al. (1988), Rowe et al. (1996), Fos (1998); *Co: MACB 103327; Ma: Rowe et al. (1996), Fos (1998).

Phlyctis argena (Ach.) Flot. - Ca: Barreno et al. (1988), Rowe et al. (1996), Fos (1998); *Co: MACB 103374; Gr: Egea et al. (1982); H: Fos (1998); J: Aragón et al. (2006a); Ma: Rowe et al. (1996), Seaward \& Arvidson (1997).

Physcia adscendens (Fr.) H. Olivier-Al: Llimona (1974), Egea \& Llimona (1981a, b, 1985, 1987, 1994), Pedreño et al. (1987), Casares \& Gutiérrez (1993), Gutiérrez \& Casares (1994), Alonso \& Egea (1996); Ca: Rowe \& Egea (1987b), Barreno et al. (1988), Casares \& Rowe (1988), Rowe et al. (1996), Fos (1998), Hernández Gallego et al. (1998); *Co: MACB 103376; Gr: Casares (1988), Casares \& Llimona (1986, 1989), Alonso \& Egea (1996), Fos (1998); H: Rowe et al. (1982), Fos (1998); J: Pedreño et al. (1987), Aragón \& Rico (1997), Aragón et al. (2004c, 2006a); Ma: Martín-Osorio (1988), Rowe et al. (1996).

Physcia aipolia (Ehrh. ex Humb.) Fürnr. - Al: Alonso \& Egea (1996); Ca: Rowe \& Egea (1986), Barreno et al. (1988), Rowe et al. (1996), Fos (1998); *Co: MACB 103378; Gr: Pedreño et al. (1987), Fos (1998); J: Pedreño et al. (1987), Aragón \& Rico (1997), Aragón et al. (2004c, 2006a); Ma: Martín-Osorio (1988), Rowe et al. (1996), Fos (1998); Se: Barras (1899), Rowe \& Egea (1985a, b, 1987a).

Physcia biziana (A. Massal.) Zahlbr. - Ca: Fos (1998); *Co: MACB 103380; Gr: Fos (1998); J: Pedreño et al. (1987), Aragón \& Rico (1997), Aragón et al. (2006a).

Physcia biziana var. leptophylla Vězda - Ca: Casares \& Rowe (1988). Physcia caesia (Hoffm.) Hampe ex Fürnr. - Al: Egea \& Llimona (1981a), Pedreño et al. (1987); Ca: Rowe et al. (1996); *Co: MACB 103381; Gr: Colmeiro (1867); J: Aragón \& Rico (1997); Se: Rowe \& Egea (1985b, 1987a).

Physcia clementei (Turner) Lynge - Ca: Colmeiro (1867); J: Pedreño et al. (1987), Aragón \& Rico (1997); Se: Colmeiro (1867).

Physcia dimidiata (Arnold) Nyl. - Ca: Rowe \& Egea (1986, 1988); Gr: Casares \& Llimona (1989).

Physcia dubia (Hoffm.) Lettau - Al: Egea \& Llimona (1981a, 1987), Pedreño et al. (1987); Ca: Rowe et al. (1996), Fos (1998); Gr: Casares \& Llimona (1982), Egea et al. (1982), Pedreño et al. (1987); Ma: Rowe et al. (1996); Se: Rowe \& Egea (1985b).

Physcia insignis Mereschk. - Al: Egea \& Llimona (1981a, b).

Physcia leptalea (Ach.) DC. - Al: Colmeiro (1867), Pedreño et al. (1987); Ca: Colmeiro (1867), Abbayes (1945), Barreno et al. (1988), Fos (1998), Hernández Gallego et al. (1998); *Co: MACB 103382; Gr: Colmeiro (1867), Fos (1998); H: Rowe et al. (1982); J: Pedreño et al. (1987), Aragón \& Rico (1997), Aragón et al. (2004c, 2006a); Ma: Martín-Osorio (1988), Fos (1998).

Physcia magnussonii Frey - Al: Egea \& Llimona (1981a, 1987), Pedreño et al. (1987); Ca: Rowe et al. (1996); Gr: Egea et al. (1982), 
Pedreño et al. (1987), Casares (1988), Casares \& Llimona (1986, 1989).

Physcia stellaris (L.) Nyl. - Al: Colmeiro (1867); Ca: Rowe et al. (1982), Fos (1998); Gr: Colmeiro (1867), Sampaio (1917), Pedreño et al. (1987), Fos (1998); J: Pedreño et al. (1987), Aragón et al. (2006a); Ma: Colmeiro (1867), Barras (1896); H: Rowe et al. (1982); Se: Barras (1896), Silvestre \& Rowe (1982).

Physcia tenella (Scop.) DC. - Al: Colmeiro (1867), Egea \& Llimona (1981a, 1987), Pedreño et al. (1987); Ca: Colmeiro (1867), Rowe et al. (1996), Fos (1998); Gr: Colmeiro (1867), Sampaio (1917), Casares (1988), Casares \& Llimona (1986, 1989), Fos (1998); J: Pedreño et al. (1987), Aragón et al. (2004c, 2006a); Ma: Rowe et al. (1996).

**Physcia tribacia (Ach.) Nyl. - Co: MACB 103385.

Physcia tribacioides Nyl. - Ca: Fos (1998).

Physconia detersa (Nyl.) Poelt - Ca: Fos (1998).

Physconia distorta (With.) J. R. Laundon - Al: Egea \& Llimona (1987); Ca: Colmeiro (1867), Barreno et al. (1988), Rowe et al. (1996), Fos (1998); Gr: Colmeiro (1867), Pedreño et al. (1987); H: Rowe et al. (1982); Ma: Martín-Osorio (1988), Rowe et al. (1996), Fos (1998), Hernández Gallego et al. (1998); Se: Rowe \& Egea (1985b).

Physconia enteroxantha (Nyl.) Poelt - Al: Egea \& Llimona (1981a, 1987), Pedreño et al. (1987); Ca: Rowe \& Egea (1986, 1988), Barreno et al. (1988), Rowe et al. (1996), Fos (1998), Hernández Gallego et al. (1998); *Co: MACB 103386; Gr: Abbayes (1945); H: Fos (1998); Ma: Rowe et al. (1996), Fos (1998).

Physconia farrea (Ach.) Poelt - Al: Egea \& Llimona (1981a, 1987).

Physconia grisea (Lam.) Poelt-Al: Pedreño et al. (1987); Ma: Rowe et al. (1996).

Physconia grisea subsp. lilacina (Arnold) Poelt - Al: Egea \& Llimona (1981a, 1987), Pedreño et al. (1987); Gr: Casares (1988), Casares \& Llimona (1989).

Physconia muscigena (Ach.) Poelt - Ca: Fos (1998); *Co: MACB 103884; Gr: Casares \& Llimona (1982), Egea et al. (1982).

Physconia perisidiosa (Erichsen) Moberg - Al: Pedreño et al. (1987); Ca: Rowe et al. (1996), Fos (1998); *Co: MACB 103390; Gr: Pedreño et al. (1987), Casares \& Llimona (1989), Fos (1998); J: Pedreño et al. (1987); Ma: Rowe et al. (1996).

Physconia servitii (Nádv.) Poelt - Ca: Fos (1998); Gr: Fos (1998).

Physconia subpulverulenta (Szatala) Poelt - Ca: Barreno et al. (1988), Rowe et al. (1996), Fos (1998).

Physconia venusta (Ach.) Poelt-Al: Egea \& Llimona (1981a), Pedreño et al. (1987); Ca: Colmeiro (1867), Rowe \& Egea (1987b), Barreno et al. (1988), Rowe et al. (1996), Hernández Gallego et al. (1998); *Co: MACB 103395; Gr: Colmeiro (1867), Casares \& Llimona (1986), Casares (1988); J: Pedreño et al. (1987), Aragón \& Rico (1997); Ma: Colmeiro (1867), Abbayes (1945), MartínOsorio (1988).

Physconia venusta subsp. subaquila (Nyl.) Clauzade \& Cl. Roux Ca: Fos (1998).

Piccolia ochrophora (Nyl.) Hafellner - Ca: Rowe et al. (1996).

Placidiopsis cinerascens (Nyl.) Breuss - Gr: Prieto et al. (2010); Ma: Prieto et al. (2010).

Placidiopsis custnani (A. Massal.) Körb. - Ma: Prieto et al. (2010).

Placidiopsis subtrachytica (B. de Lesd.) Zschacke - Gr: Casares \& Llimona $(1984,1986)$.

Placidiopsis tenella (Nyl.) Zahlbr. - Al: Gutiérrez \& Casares (1994), Guerra et al. (1995).

Placidium adami-borosi Szatala - J: Prieto et al. (2010).
Placidium fingens (Breuss) Breuss - Ma: Seaward \& Arvidson (1997).

Placidium imbecillum (Breuss) Breuss - Ca: Prieto et al. (2010); J: Prieto et al. (2010) Ma: Prieto et al. (2010).

Placidium lachneum (Ach.) B. de Lesd. - Ca: Colmeiro (1867); Gr: Egea et al. (1982), Casares (1988).

Placidium lacinulatum (Ach.) Breuss - J: Prieto et al. (2010); Ma: Seaward \& Arvidson (1997), Prieto et al. (2010).

Placidium michelii A. Massal. - Ca: Prieto et al. (2010).

Placidium pilosellum (Breuss) Breuss - Al: Prieto et al. (2010); Ca: Prieto et al. (2010); Gr: Casares \& Gutiérrez (1993), Prieto et al. (2010); H: Prieto et al. (2010); J: Prieto et al. (2010); Ma: Seaward \& Arvidson (1997), Prieto et al. (2010).

Placidium rufescens (Ach.) A. Massal. -Al: Egea \& Llimona (1981a), Casares \& Gutiérrez (1993), Gutiérrez \& Casares (1994), Martínez-Sánchez et al. (1994), Prieto et al. (2010); Ca: Prieto et al. (2010); *Co: MACB 103884; Gr: Prieto \& Espinosa (1971), Werner (1979), Casares \& Llimona (1982), Casares \& Gutiérrez (1993), Prieto et al. (2010); J: Prieto et al. (2010); Ma: Prieto et al. (2010).

Placidium semaforonense (Breuss) Breuss - Al: Prieto et al. (2010); J: Prieto et al. (2010); Ma: Seaward \& Arvidson (1997), Prieto et al. (2010).

Placidium subrufescens (Breuss) Breuss - J: Prieto et al. (2010).

Placidium tenellum (Breuss) Breuss - Al: Prieto et al. (2010); Ma: Prieto et al. (2010).

Placidium squamulosum (Ach.) Breuss -Al: Egea \& Llimona (1994), Alonso \& Egea (1996), Prieto et al. (2010); Ca: Rowe et al. (1996), Ariño et al. (1997), Prieto et al. (2010): *Co: MACB 103885; Gr: Alonso \& Egea (1996), Prieto et al. (2010); J: Prieto et al. (2010); Ma: Seaward \& Arvidson (1997), Prieto et al. (2010); Se: Ariño et al. (1997).

Placocarpus schaereri (Fr.) Breuss - *Co: MACB 103325; Gr: Casares \& Llimona (1984, 1986), Casares (1986).

Placolecis opaca (Dufour) Hafellner-Al: Alonso \& Egea (1996); Ca: Rowe et al. (1996); *Co: MACB 103326; Gr: Casares \& Llimona (1989).

Placynthiella icmalea (Ach.) Coppins \& P. James - J: Aragón et al. (2006a).

Placynthiella uliginosa (Schrad.) Coppins \& P. James - J: Aragón et al. (2006a); Ma: Herrera et al. (2007).

Placynthium asperellum (Ach.) Trevis. - *Co: MACB 103438; J: Aragón et al. (2006b), Burgaz (2011a, b); Gr: Burgaz (2010, 2011a, b); Ma: Burgaz (2010, (2011a, b).

Placynthium filiforme (Garov.) M. Choisy - J: Aragón et al. (2006b).

Placynthium hungaricum Gyeln. - Gr: Burgaz (2010, 2011a, b); J: Aragón et al. (2006b), Burgaz (2010, 2011a, b); Ma: Burgaz (2010, 2011a, b).

Placynthium nigrum (Huds.) Gray -Al: Casares \& Gutiérrez (1993), Egea \& Llimona (1994), Gutiérrez \& Casares (1994), Alonso \& Egea (1996), Burgaz \& Martínez (2002); Burgaz (2010, 2011a, b); Ca: Casares \& Rowe (1988), Alonso \& Egea (1996), Rowe et al. (1996), Burgaz \& Martínez (2002), Burgaz (2010); Co: Burgaz \& Martínez (2002), Burgaz (2010, 2011a, b); Gr: Casares (1988), Casares \& Llimona (1986, 1989), Martínez-Sánchez et al. (1994), Alonso \& Egea (1996), Burgaz \& Martínez (2002), Burgaz (2010, 2011a, b); H: Burgaz (2011a, b); J: Burgaz \& Martínez (2002), Burgaz (2010, 2011a, b); Ma: Burgaz \& Martínez (2002), Burgaz (2010, 2011a, b).

Placynthium subradiatum (Nyl.) Arnold -Al: Alonso \& Egea (1996), Burgaz (2011a, b); Ca: Burgaz (2010, 2011a, b); Co: Burgaz 
(2010, 2011a, b); Gr: Burgaz (2010, 2011a, b); J: Aragón et al. (2006a); Burgaz (2010, 2011a, b); Ma: Seaward (1983), Seaward \& Arvidson (1997), Burgaz (2010, 2011a, b).

Placynthium tantaleum (Hepp) Hue - Ca: Burgaz (2010, 2011a, b); Co: Burgaz (2011a, b); Gr: Burgaz (2010, 2011a, b); H: Burgaz (2011a, b); J: Burgaz (2010, 2011a, b).

Placynthium tremniacum (A. Massal.) Jatta - Al: Burgaz (2011a, b); Ca: Burgaz (2010, 2011a, b); Gr: Burgaz \& Martínez (2002), Burgaz (2010, 2011a, b); J: Burgaz \& Martínez (2002), Burgaz (2010, 2011a, b); Ma: Burgaz (2010, 2011a, b).

Platismatia glauca (L.) W. L. Culb. \& C. F. Culb. - Ca: Egea \& Rowe (1987), Hernández Gallego et al. (1998); H: Rowe et al. (1982); J: Aragón \& Rico (1997), Aragón et al. (2006a); Ma: Colmeiro (1867), Martín-Osorio (1988), Seaward \& Arvidson (1997), Fos (1998).

Pleopsidium flavum Korb. -Al: Egea \& Llimona (1981a, 1983, 1987); Gr: Prieto \& Espinosa (1971), Casares \& Llimona (1982), Egea et al. (1982), Silvestre \& Rowe (1982), Egea \& Llimona (1983).

Pleurosticta acetabulum (Neck.) Elix \& Lumbsch - J: Aragón \& Rico (1997), Aragón et al. (2006a); Se: Lázaro Ibiza (1898b).

\#Polyblastia nidulans (Stenh.) Arnold - Ca: Casares \& Rowe (1988).

Polyblastia sepulta A. Massal. - Ca: Casares \& Rowe (1988); Gr: Casares \& Llimona (1984).

Polyblastia theleodes (Sommerf.) Th. Fr. - Gr: Egea et al. (1982).

Polyblastiopsis subericola B. de Lesd. - Ca: Fos (1998).

Polychidium muscicola (Sw.) Gray - Ca: Rowe \& Egea (1988), Burgaz (2011a, b); H: Rowe \& Egea (1987a), Burgaz (2011a, b); Ma: Seaward \& Arvidson (1997); Se: Burgaz (2011a, b).

\#Polycoccum crassum Vězda - Ma: Martínez \& Hafellner (1998), Martínez (1999).

Polysporina cyclocarpa (Anzi) Vězda - Gr: Casares \& Llimona (1989).

Polysporina simplex (Taylor) Vězda - Al: Egea \& Llimona (1981a, b, 1983, 1987), Casares \& Gutiérrez (1993); Ca: Rowe \& Egea (1986, 1988), Egea \& Rowe (1987), Rowe et al. (1996); Gr: Sampaio (1917), Egea et al. (1982); Se: Rowe \& Egea (1985b).

Porina aenea (Wallr.) Zahlbr. - Ca: Fos (1998).

Porina borreri (Trevis.) D. Hawksw. \& P. James - Ca: Ariño et al. (1995), Fos (1998).

Porina chlorotica (Ach.) Müll. Arg. - Ca: Rowe \& Egea (1987b), Rowe et al. (1996); Gr: Colmeiro (1867), Egea et al. (1982).

Porina guentheri (Flot.) Zahlbr. - Gr: Sampaio (1917).

Porina linearis (Leight.) Zahlbr. - Al: Egea \& Llimona (1984), Alonso \& Egea (1996); Ca: Ariño et al. (1997); Gr: Casares \& Llimona (1984), Alonso \& Egea (1996); Se: Ariño et al. (1997).

Porina oleriana (A. Massal.) Lettau - Gr: Casares \& Llimona (1984).

Porpidia albocaerulescens (Wulfen) Hertel \& Knoph var. albocaerulescens - Al: Egea \& Llimona (1981a); Gr: Colmeiro (1867).

Porpidia cinereoatra (Ach.) Hertel \& Knoph - Ca: Rowe \& Egea (1986), Rowe et al. (1996).

Porpidia crustulata (Ach.) Hertel \& Knoph - Ca: Rowe \& Egea (1986); ${ }^{*}$ Co: 106698; Se: Rowe \& Egea (1987a).

Porpidia macrocarpa (DC.) Hertel \& A. J. Schwab - Al: Egea \& Llimona (1987, 1994); Ca: Colmeiro (1867), Rowe \& Egea (1986, 1987b, 1988), Rowe et al. (1996); *Co: 106699; H: Rowe \& Egea (1987a); Se: Rowe \& Egea (1987a).

Porpidia platycarpoides (Bagl.) Hertel - Ca: Rowe \& Egea (1988).

Porpidia speirea (Ach.) Kremp. - Gr: Colmeiro (1867), Egea et al. (1982).

Porpidia trullisata (Kremp.) Körb. - Ca: Rowe et al. (1996).

Porpidia zeoroides (Anzi) Knoph \& Hertel - Ca: Rowe \& Egea (1987b).
\#Pronectria pilosa Etayo \& López Silanes - Ma: López de Silanes et al. (2009).

\#Pronectria robergei (Mont. \& Desm.) Lowen - Ma: Rowe et al. (1996).

Protoblastenia calva (Dicks.) Zahlbr. - Ca: Casares \& Rowe (1988), Rowe et al. (1996); *Co: MACB 103886; Gr: Casares \& Llimona $(1986,1989)$.

Protoblastenia incrustans (DC.) J. Steiner - Ca: Casares \& Rowe (1988); Gr: Casares \& Llimona (1989); Ma: Herrera et al. (2007).

Protoblastenia rupestris (Scop.) J. Steiner - Gr: Casares \& Llimona (1989).

Protopannaria pezizoides (Weber ex F. H. Wigg.) P. M. Jørg. \& S. Ekman - Gr: Carballal \& López de Silanes (2010); Ma: Rowe et al. (1996).

Protoparmelia badia (Hoffm.) Hafellner - Al: Colmeiro (1867), Egea \& Llimona (1981a, 1983, 1987); Gr: Egea et al. (1982).

Protoparmelia montagnei (Fr.) Sancho \& A. Crespo-Al: Egea \& Llimona (1981a, b, 1983, 1984, 1994), Barbero et al. (2006); Ca: Rowe \& Egea $(1986,1988)$, Egea \& Rowe (1987); *Co: MACB 103330; Ma: Herrera et al. (2007).

Protoparmelia nephaea (Sommerf.) R. Sant. - Se: Rowe \& Egea (1987a).

Protoparmelia psarophana (Nyl.) Sancho \& Crespo -Al: Egea \& Llimona (1981a, b, 1987), Barbero et al. (2006); Ca: Rowe \& Egea (1986, 1988), Egea \& Rowe (1987); Ma: Herrera et al. (2007).

Pseudephebe minuscula (Nyl. ex Arnold) Brodo \& D. Hawksw. - Gr: Degelius (1966), Casares \& Llimona (1982), Egea et al. (1982).

Pseudevernia furfuracea (L.) Zopf - Ca: Colmeiro (1867), Egea \& Rowe (1987), Barreno et al. (1988); *Co: MACB 105162; Gr: Colmeiro (1867); H: Fos (1998); J: Aragón \& Rico (1997), Aragón et al. (2006a); Ma: Colmeiro (1867), Sampaio (1917), Martín-Osorio (1988).

Psora albilabra (Dufour) Körb. subsp. albilabra - Al: Casares \& Gutiérrez (1993), Gutiérrez \& Casares (1994); Gr: Casares (1988), Casares \& Llimona (1986, 1989), Casares \& Gutiérrez (1993), Martínez-Sánchez et al. (1994).

Psora crenata (Taylor) Reinke - Al: Llimona (1974), Martínez-Sánchez et al. (1994); Gr: Martínez-Sánchez et al. (1994).

Psora decipiens (Hedw.) Hoffm. -Al: Casares \& Gutiérrez (1993), Gutiérrez \& Casares (1994), Alonso \& Egea (1996); Ca: Rowe et al. (1996); *Co: MACB 103446; Gr: Colmeiro (1867), Casares \& Llimona (1989), Casares \& Gutiérrez (1993), Martínez-Sánchez et al. (1994), Alonso \& Egea (1996); J: Aragón \& Rico (1997); Ma: Colmeiro (1867); Se: Barras (1899).

Psora saviczii (Tomin) Follmann \& A. Crespo - Al: Casares \& Gutiérrez (1993), Egea \& Llimona (1994), Gutiérrez \& Casares (1994), Guerra et al. (1995); Gr: Casares \& Gutiérrez (1993), MartínezSánchez et al. (1994).

Psora testacea Hoffm. - Ca: Rowe et al. (1996); Gr: Colmeiro (1867), Casares (1988), Casares \& Llimona (1989), Rowe et al. (1996); J: Aragón \& Rico (1997); Ma: Colmeiro (1867), Rowe et al. (1996), Seaward \& Arvidson (1997).

Psora vallesiaca (Schaer.) Timdal -Al: Egea \& Llimona (1994); *Co: MACB 103447; J: Aragón \& Rico (1997); Ma: Seaward \& Arvidson (1997).

Psorinia conglomerata (Ach.) Gotth. Schneid. - Gr: Colmeiro (1867).

Psoroglaena stigonemoides (Orange) Henssen - J: Aragón \& Sarrión (2003).

Psoroma hypnorum (Vahl) Gray - Ca: Colmeiro (1867); Gr: Colmeiro (1867), Egea et al. (1982). 
Psorotichia diffracta (Nyl.) Forssell - Gr: Casares \& Llimona (1989). Psorotichia frustulosa Anzi -Al: Egea \& Llimona (1994).

Psorotichia montinii (A. Massal.) Forssell - Al: Gutiérrez \& Casares (1994).

Psorotichia schaereri (A. Massal.) Arnold - Gr: Casares \& Llimona (1989).

Pterygiopsis affinis (A. Massal.) Henssen - Ma: Herrera et al. (2007).

Punctelia borreri (Sm.) Krog - Ca: Colmeiro (1867), Hernández Gallego et al. (1998).

Punctelia reddenda (Stirt.) Krog - Ca: Fos (1998).

Punctelia subrudecta (Nyl.) Krog - Ca: Barreno et al. (1988), Fos (1998), Crespo et al. (2004); *Co: MACB 103887; H: Fos (1998), Crespo et al. (2004); J: Aragón \& Rico (1997); Ma: Fos (1998).

Pyrenocollema halodytes (Nyl.) R. C. Harris - Gr: Casares \& Llimona (1983, 1984); Ma: Herrera et al. (2007).

Pyrenodesmia alociza (A. Massal.) Arnold - Ca: Casares \& Rowe (1988), Rowe et al. (1996); Gr: Egea (1984), Casares \& Llimona (1989).

Pyrenodesmia chalybaea (Fr.) A. Massal. - Gr: Sampaio (1917), Egea (1984), Casares \& Llimona (1986), Casares (1988); Ma: Seaward \& Arvidson (1997), Herrera et al. (2007); Se: Puertas et al. (1994).

Pyrenodesmia variabilis (Pers.) A. Massal. - Al: Egea \& Llimona (1994), Gutiérrez \& Casares (1994), Alonso \& Egea (1996); Ca: Sampaio (1917), Casares \& Rowe (1988), Ariño et al. (1995), Rowe et al. (1996); Gr: Colmeiro (1867), Egea (1984), Casares \& Llimona (1986), Alonso \& Egea (1996); Se: Ariño et al. (1997).

Pyrenopsis triptococca Nyl. -Al: Llimona \& Egea (1985), Egea \& Llimona (1994); Ca: Rowe \& Egea (1986, 1988); Se: Llimona \& Egea (1985), Rowe \& Egea (1985a, 1987a).

Pyrenula chlorospila (Nyl.) Arnold - Ca: Fos (1998).

Pyrrhospora lusitanica (Räsänen) Hafellner - J: Aragón et al. (2006a).

Pyrrhospora quernea (Dicks.) Körb. - Ca: Barreno et al. (1988), Fos (1998); H: Fos (1998); J: Aragón et al. (2006a).

Pyxine subcinerea Stirt. - Ca: Fos (1998).

Ramalina breviuscula Nyl. -Al: Llimona (1975); Ma: Herrera et al. (2007).

Ramalina calicaris (L.) Röhl. - Ca: Sampaio (1917), Barreno et al. (1988), Fos (1998), Arroyo (1991); *Co: MACB 103267; H: Fos (1998); Ma: Martín-Osorio (1988), Fos (1998).

Ramalina canariensis J. Steiner - Al: Arroyo (1991); Ca: Barreno et al. (1988), Fos (1998); H: Arroyo (1991), Fos (1998); Ma: Seaward \& Arvidson (1997).

Ramalina capitata (Ach.) Nyl. -Al: Egea \& Llimona (1981a, b, 1983, 1987), Arroyo (1991), Arroyo et al. (1991); Ca: Arroyo (1991); Gr: Arroyo (1991); Se: Rowe \& Egea (1985b), Arroyo (1991), Arroyo et al. (1991).

Ramalina capitata var. protecta (H. Magn.) Nimis - Al: Egea \& Llimona (1981a, 1983, 1987), Arroyo et al. (1991); Ca: Rowe \& Egea (1987b), Rowe et al. (1996); Gr: Casares \& Llimona (1982), Egea et al. (1982), Arroyo et al. (1991); H: Arroyo et al. (1991).

Ramalina clementeana Llimona \& Werner - Al: Llimona \& Werner (1975), Egea \& Llimona (1983, 1984, 1994), Arroyo (1991); Ma: Herrera et al. (2007).

Ramalina digitellata Nyl. - Al: Egea \& Llimona (1981a, 1983, 1987, 1994), Arroyo et al. (1991); Ca: Rowe \& Egea (1986), Arroyo et al. (1991); *Co: MACB 103268; Gr: Egea et al. (1982); Se: Rowe \& Egea (1985a, b, 1987a); Arroyo et al. (1991).

Ramalina farinacea (L.) Ach. - Al: Colmeiro (1867), Arroyo (1991); Ca: Colmeiro (1867), Sampaio (1917), Rowe \& Egea (1986), Egea
\& Rowe (1987), Barreno et al. (1988), Arroyo (1991), Fos (1998), Hernández Gallego et al. (1998); *Co: MACB 103269; Gr: Fos (1998); H: Arroyo (1991), Fos (1998); J: Sampaio (1917), Arroyo (1991), Aragón \& Rico (1997), Aragón et al. (2006a); Ma: Colmeiro (1867), Martín-Osorio (1988), Arroyo (1991), Fos (1998); Se: Colmeiro (1867), Lázaro Ibiza (1898a)

Ramalina fastigiata (Pers.) Ach. - Ca: Colmeiro (1867), Barreno et al. (1988), Arroyo (1991), Fos (1998); Co: Colmeiro (1867); H: Fos (1998); J: Arroyo (1991), Aragón \& Rico (1997); Ma: Colmeiro (1867), Barras (1896), Martín-Osorio (1988), Fos (1998); Se: Barras (1896), Lázaro Ibiza (1898a), Silvestre \& Rowe (1982).

Ramalina fraxinea (L.) Ach. - Ca: Colmeiro (1867), Barreno et al. (1988), Arroyo (1991), Fos (1998); *Co: MACB 103275; Gr: Fos (1998); J: Colmeiro (1867), Arroyo (1991), Aragón \& Rico (1997); Ma: Colmeiro (1867); Se: Colmeiro (1867), Lázaro Ibiza (1898a).

Ramalina huei Harm. - Ca: Arroyo (1991).

Ramalina inflata subsp. australis G. N. Stevens - Ca: Rowe \& Egea (1986), Barreno et al. (1988), Fos (1998).

Ramalina lacera (With.) J. R. Laundon - Al: Arroyo (1991); Ca: Barreno et al. (1988), Fos (1998).

Ramalina obtusata (Arnold) Bitter - Gr: Casares \& Llimona (1982).

Ramalina panizzei de Not. - Al: Arroyo (1991).

Ramalina pollinaria (Westr.) Ach. - Al: Colmeiro (1867); Ca: Colmeiro (1867), Sampaio (1917); Gr: Colmeiro (1867); J: Aragón et al. (2006a); Se: Lázaro Ibiza (1898a).

Ramalina polymorpha (Lilj.) Ach. - Al: Colmeiro (1867), Llimona (1975), Silvestre \& Rowe (1982); Ca: Colmeiro (1867); Gr: Colmeiro (1867)

Ramalina requienii (De Not.) Jatta -Al: Egea \& Llimona (1983, 1994), Arroyo (1991), Casares \& Gutiérrez (1993), Gutiérrez \& Casares (1994); Ca: Rowe \& Egea (1986), Arroyo (1991).

Ramalina roesleri (Hochst. ex Schaer.) Hue - Se: Rowe \& Egea (1985b).

Ramalina rosacea (A. Massal.) Hepp - Al: Abbayes (1945), Llimona (1975), Egea \& Llimona (1981b, 1983, 1984, 1987), Arroyo (1991), Egea \& Llimona (1994); Ca: Rowe \& Egea (1986), Arroyo (1991); Ma: Seaward \& Arvidson (1997), Herrera et al. (2007).

Ramalina siliquosa (Huds.) A. L. Sm. - Ca: Rowe \& Egea (1986).

Ramalina subfarinacea (Nyl. ex Cromb.) Nyl. - Al: Egea \& Llimona (1981a, 1983), Arroyo (1991); Ca: Rowe \& Egea (1986), Arroyo (1991); Ma: Sampaio (1917); Se: Rowe \& Egea (1985b).

**Ramalina subgeniculata Nyl. - Co: MACB 106501.

Ramalina subpusilla (Nyl.) Krog \& Swinscow - Ca: Arroyo (1991), Fos (1998).

Ramalina tingitana Salzm. - Al: Abbayes (1945), Egea \& Llimona (1983, 1984, 1994)

Ramboldia insidiosa (Th. Fr.) Hafellner - J: Aragón et al. (2004c).

Ramboldia stuartii (Hampe) Kantvilas \& Elix - J: Aragón et al. (2004c).

Ramonia subsphaeroides (Tav.) Vězda - Ca: López de Silanes (2004).

Rhizocarpon disporum (Nägeli ex Hepp) Müll. Arg. -Al: Egea \& Llimona (1981a, 1983, 1987); Gr: Egea et al. (1982).

Rhizocarpon distinctum Th. Fr. - Al: Egea \& Llimona (1981a, 1983, 1987).

Rhizocarpon effiguratum (Anzi) Th. Fr. - Gr: Degelius (1966).

Rhizocarpon epispilum (Nyl.) Zahlbr. - Al: Egea \& Llimona (1981a, 1983); Ca: et al. (1996).

Rhizocarpon geminatum Körb. - Gr: Pereira et al. (1987).

Rhizocarpon geographicum (L.) DC. - Al: Egea \& Llimona (1981a, b, 1983, 1987, 1994); Ca: Colmeiro (1867), Crespí (1930), Rowe 
\& Egea (1986, 1988), Egea \& Rowe (1987), Rowe et al. (1996); *Co: 106700; Gr: Colmeiro (1867), Sampaio (1917), Crespí (1930), Degelius (1966), Prieto \& Espinosa (1971), Casares \& Llimona (1982), Egea et al. (1982); Silvestre \& Rowe (1982); H: Rowe \& Egea (1987a); J: Colmeiro (1867), Crespo et al. (1976); Se: Rowe \& Egea (1985a, b, 1987a).

Rhizocarpon granatense (Clemente) Zahlbr. - Gr: Colmeiro (1867).

Rhizocarpon lecanorinum Anders - Al: Egea \& Llimona (1981a, 1987); Gr: Egea et al. (1982).

Rhizocarpon lusitanicum (Nyl.) Arnold-Al: Egea \& Llimona (1983, 1994).

Rhizocarpon malenconianum (Llimona \& Werner) Hafellner \& Mayrhofer - Al: Llimona (1974), Casares \& Gutiérrez (1993); Gutiérrez \& Casares (1994).

Rhizocarpon oportense (Vain.) Räsänen - Ca: Rowe \& Egea (1986, 1988); Se: Rowe \& Egea (1985b)

Rhizocarpon polycarpum (Hepp) Th. Fr. - Ca: Rowe \& Egea (1986).

Rhizocarpon richardii (Lamy ex Nyl.) Zahlbr. - Al: Egea \& Llimona (1981a, 1983, 1987); Ca: Rowe \& Egea (1986), Egea \& Rowe (1987); H: Rowe \& Egea (1987a); Se: Rowe \& Egea (1985a, b, 1987a).

Rhizocarpon saanaense Räsänen - Al: Egea \& Llimona (1981a).

Rhizocarpon simillimum (Anzi) Lettau. -Al: Egea \& Llimona (1981a); Ca: Rowe \& Egea (1987b), Rowe et al. (1996); Gr: Colmeiro (1867).

Rhizocarpon sublucidum Räsänen-Al: Egea \& Llimona (1981a, 1983, 1987); Gr: Degelius (1966), Egea et al. (1982).

Rhizocarpon superficiale (Schaer.) Malme - Gr: Degelius (1966), Prieto \& Espinosa (1971), Casares \& Llimona (1982), Egea et al. (1982).

Rhizocarpon tetrasporum Runemark - Ca: Rowe \& Egea (1986); H: Rowe \& Egea (1987a); Se: Rowe \& Egea (1985a, 1987a).

Rhizocarpon tinei Runemark - Al: Egea \& Llimona (1981a, 1983); Ca: Rowe \& Egea (1986).

Rhizocarpon viridiatrum (Wulfen) Körb. - Ca: Egea \& Rowe (1987); Gr: Casares \& Llimona (1982).

Rhizoplaca chrysoleuca (Sm.) Zopf - Al: Egea \& Llimona (1981a, 1987); Gr: Sampaio (1917), Casares \& Llimona (1982), Egea et al. (1982).

Rhizoplaca melanophthalma (DC.) Leuckert - Al: Egea \& Llimona (1981a, 1987); Ca: Colmeiro (1867); Gr: Colmeiro (1867), Sampaio (1917), Prieto \& Espinosa (1971), Casares \& Llimona (1982), Egea et al. (1982), Lumbsch et al. (1991); J: Colmeiro (1867

Rhizoplaca melanophthalma var. obscura (J. Steiner) Leuckert \& Poelt - Gr: Degelius (1966).

Rhizoplaca peltata (Ramond) Leuckert \& Poelt - Al: Egea \& Llimona (1981a, 1987); Gr: Prieto \& Espinosa (1971), Casares \& Llimona (1982), Egea et al. (1982).

Rimularia insularis (Nyl.) Rambold \& Hertel - Al: Egea \& Llimona (1987); Ca: Rowe \& Egea (1987b), Rowe et al. (1996).

Rinodina alba Metzler ex Arnold -Al: Egea \& Llimona (1981b, 1983, 1987, 1994), Giralt (2010); Ca: Rowe \& Egea (1986), Giralt \& Barbero (1995); Ma: Herrera et al. (2007); Se: Rowe \& Egea (1985b).

Rinodina anomala (Zahlbr.) H. Mayrhofer \& Giralt - J: Aragón et al. (2004c), Giralt (2010).

Rinodina archaea (Ach.) Arnold - Ca: Fos (1998), Giralt (2010); H: Fos (1998); J: Aragón et al. (2006a), Giralt (2010).

Rinodina atrocinerea (Sm. ex Hook.) Körb. - Al: Egea \& Llimona (1983, 1987); Ca: Rowe \& Egea (1986); Gr: Werner (1979); H:
Rowe \& Egea (1987a); Ma: Navarro-Rosinés et al. (1998), Giralt (2010); Se: Giralt (2010).

Rinodina atrocinerella (Nyl.) Boistel - Al: Egea \& Llimona (1981a, 1983, 1987); Gr: Egea et al. (1982).

Rinodina beccariana Bagl. - Al: Egea \& Llimona (1983, 1994), Giralt \& Barbero (1995), Giralt (2010); Ca: Rowe \& Egea (1986), Egea \& Rowe (1987), Rowe et al. (1996), Giralt (2010); Ma: Herrera et al. (2007); Se: Giralt (2010).

Rinodina beccariana var. lavicola (J. Steiner) Matzer \& H. Mayrhofer - Ca: Rowe \& Egea (1986), Egea \& Rowe (1987), Giralt (2010).

Rinodina bischoffii (Hepp) A. Massal. -Al: Egea \& Llimona (1994), Alonso \& Egea (1996), Giralt \& Llimona (1997), Giralt (2010); Ca: Casares \& Rowe (1988); Gr: Casares (1988), Casares \& Llimona (1986, 1989), Giralt \& Barbero (1995), Giralt (2010); Ma: Herrera et al. (2007), Giralt (2010); Se: Ariño et al. (1997), Giralt (2010).

Rinodina calcarea (Hepp ex Arnold) Arnold - J: Giralt (2010).

Rinodina cana (Arnold) Arnold - Ca: Rowe \& Egea (1988), Giralt (2010); Se: Rowe \& Egea (1987a).

Rinodina capensis Hampe - Ca: Fos (1998), Giralt (2010); *Co: MACB 103890; H: Fos (1998), Giralt (2010); J: Aragón et al. (2006a).

Rinodina colletica (Flörke) Arnold - Gr: Werner (1979).

Rinodina colobina (Ach.) Th. Fr. - Ca: Giralt (2010); J: Aragón et al. (2006a); Ma: Herrera et al. (2007), Giralt (2010).

Rinodina confragosa (Ach.) Körb. - Al: Egea \& Llimona (1981a, 1987), Giralt \& Barbero (1995), Giralt (2010); Ca: Rowe \& Egea (1986, 1988), Rowe et al. (1996), Giralt (2010); Gr: Werner (1979), Giralt \& Barbero (1995), Giralt (2010); H: Rowe \& Egea (1987a).

Rinodina dalmatica Zahlbr. - Ca: Fos (1998), Fos et al. (2000), Giralt (2010); J: Fos et al. (2000), Aragón et al. (2006a), Giralt (2010).

Rinodina dubyana (Hepp) J. Steiner - Ca: Giralt \& Llimona (1997), Giralt (2010); Gr: Giralt \& Llimona (1997), Giralt (2010); J: Giralt \& Llimona (1997), Giralt (2010).

Rinodina epimilvina H. Mayrhofer - Gr: Giralt (2010).

Rinodina exigua (Ach.) Gray - Ca: Colmeiro (1867), Barreno et al. (1988), Fos (1998); H: Fos (1998), Giralt (2010); J: Aragón et al. (2006a); Ma: Martín-Osorio (1988).

Rinodina furfuracea H. Magn. - J: Aragón et al. (2004c), Giralt (2010).

Rinodina gennarii Bagl. -Al: Egea \& Llimona (1981a, b, 1983, 1987), Giralt \& Llimona (1997), Giralt (2010); Ca: Ariño et al. (1995), Giralt (2010); Ma: Giralt (2010).

Rinodina griseosoralifera Coppins - Ca: Fos (1998), Giralt (2010); H: Fos (1998); Ma: Giralt (2010).

Rinodina guzzinii Jatta - Al: Alonso \& Egea (1996), Giralt \& Llimona (1997), Giralt (2010); Gr: Giralt \& Llimona (1997), Giralt (2010); Se: Giralt (2010).

Rinodina immersa (Körb.) J. Steiner - Ca: Casares \& Rowe (1988); Gr: Casares \& Llimona (1986, 1989), Giralt \& Llimona (1997), Giralt (2010); Ma: Herrera et al. (2007), Giralt (2010); Se: Giralt (2010).

Rinodina isidioides (Borrer) H. Olivier - Ca: Fos (1998), Giralt (2010).

Rinodina lecanorina (A. Massal.) A. Massal. - Ca: Casares \& Rowe (1988), Rowe et al. (1996), Giralt (2010); Gr: Casares \& Llimona (1989), Giralt \& Llimona (1997), Giralt (2010).

Rinodina llimonae Giralt \& Etayo - Ma: Giralt (2010).

Rinodina melanocarpa Müll. Arg. - Gr: Egea (1985). 
Rinodina milvina (Wahlenb.) Th. Fr. - Al: Egea \& Llimona (1981a, 1987), Egea (1985), Giralt \& Llimona (1997); Gr: Egea et al. (1982), Giralt \& Llimona (1997), Giralt (2010).

Rinodina obnascens (Nyl.) H. Olivier - Al: Egea \& Llimona (1981a, 1987), Giralt \& Llimona (1997), Giralt (2010); Ca: Rowe et al. (1996), Giralt (2010); Gr: Egea et al. (1982), Giralt \& Llimona (1997), Giralt (2010).

Rinodina occulta (Körb.) Sheard - Al: Egea \& Llimona (1981a), Giralt \& Llimona (1997), Giralt (2010).

Rinodina ocellata (Hoffm.) Arnold - Gr: Casares \& Llimona (1986), Casares (1988).

Rinodina oleae Bagl. - J: Giralt \& Mayrhofer (1995), Aragón et al. (2004c, 2006a), Giralt (2010); Ma: Seaward \& Arvidson (1997), Giralt (2010).

Rinodina plana H. Magn. - Ca: Fos (1998), Giralt (2010); *Co: MACB 103891; J: Giralt (2010).

Rinodina pruinella Bagl. - Al: Giralt \& Mayrhofer (1994), Giralt (2010); Ca: Giralt (2010).

Rinodina pyrina (Ach.) Arnold-Al: Giralt (2010); Ca: Giralt (2010); Gr: Fos (1998), Giralt (2010); J: Aragón et al. (2006a), Giralt (2010); Ma: Martín-Osorio (1988).

Rinodina rinodinoides (Anzi) H. Mayrhofer \& Scheid. - Gr: Giralt (2010); Al: Giralt \& Llimona (1997), Giralt (2010).

Rinodina roboris (Dufour ex Nyl.) Arnold - Ca: Barreno et al. (1988), Fos (1998), Giralt (2010); J: Giralt (2010); Ma: Giralt (2010).

Rinodina santorinensis J. Steiner - Al: Egea \& Llimona (1994), Giralt \& Barbero (1995), Giralt (2010).

Rinodina sauvagei Werner - Al: Llimona \& Werner (1975).

Rinodina septentrionalis Malme - Ca: Giralt (2010); H: Fos (1998); J: Giralt \& Mayrhofer (1995), Aragón et al. (2006a), Giralt (2010).

Rinodina serpentini H. Mayrhofer \& Poelt - Al: Egea (1985).

Rinodina sophodes (Ach.) A. Massal. - Ca: Fos (1998), Giralt (2010); H: Fos (1998), Giralt (2010); J: Aragón et al. (2004c), Giralt (2010); Ma: Giralt (2010).

Rinodina teichophila (Nyl.) Arnold - Al: Egea \& Llimona (1981a), Giralt \& Llimona (1997), Giralt (2010).

Rinodina trachytica (A. Massal.) Bagl. \& Carestia - Ca: Rowe \& Egea (1986, 1988), Giralt (2010); Co: Giralt (2010); H: Rowe et al. (1982), Rowe \& Egea (1987a); Se: Rowe \& Egea (1987a); Ma: Giralt (2010).

Rinodina tunicata H. Mayrhofer \& Poelt - Gr: Giralt \& Llimona (1997), Giralt (2010).

Rinodinella controversa (A. Massal.) H. Mayrhofer \& Poelt - Gr: Casares \& Llimona (1986, 1989), Alonso \& Egea (1996), Giralt (2010); Ma: Giralt (2010).

Rinodinella dubyanoides (Hepp) H. Mayrhofer \& Poelt - Al: Giralt (2010); Ma: Giralt (2010).

Roccella arnoldii Vain. - Ca: Rowe \& Egea (1986); Gr: Casares \& Llimona (1989).

Roccella canariensis Darb. -Al: Egea \& Llimona (1994); Ma: Herrera et al. (2007).

Roccella fuciformis (L.) DC. -Al: Colmeiro (1867), Egea \& Llimona (1994); Ca: Colmeiro (1867); Rowe \& Egea (1986), Egea \& Rowe (1987); Ma: Herrera et al. (2007).

Roccella phycopsis Ach. -Al: Colmeiro (1867); Abbayes (1945), Egea \& Llimona (1981b, 1983, 1984, 1994), Silvestre \& Rowe (1982), Alonso \& Egea (1996); Ca: Sampaio (1917), Rowe \& Egea (1986), Egea \& Rowe (1987); Gr: Casares \& Llimona (1989), Alonso \& Egea (1996); Ma: Herrera et al. (2007).
Roccella vicentina (Vain.) Follmann - Al: Egea \& Llimona (1983, 1984, 1994); Ca: Rowe \& Egea (1986), Egea \& Rowe (1987).

Romjularia lurida (Ach.) Timdal -Al: Egea \& Llimona (1981b), Alonso \& Egea (1996); Ca: Colmeiro (1867), Casares \& Rowe (1988); *Co: MACB 103276; Gr: Colmeiro (1867), Silvestre \& Rowe (1982), Casares \& Llimona (1989); J: Aragón \& Rico (1997); Ma: Colmeiro (1867).

Rufoplaca arenaria (Pers.) Arup et al. -Al: Egea \& Llimona (1981a, b, 1983, 1987, 1994), Egea (1984), Llimona \& Egea (1985), Alonso \& Egea (1996); Ca: Colmeiro (1867), Rowe \& Egea (1986, 1988), Ariño et al. (1995, 1997), Rowe et al. (1996); Gr: Colmeiro (1867), Degelius (1966), Egea et al. (1982), Egea (1984), Casares \& Llimona (1986, 1989); H: Rowe et al. (1982), Rowe \& Egea (1987a); J: Colmeiro (1867); Ma: Colmeiro (1867), Herrera et al. (2007); Se: Egea (1984), Rowe \& Egea (1985a, b, 1987a).

Rusavskia elegans (Link) S. Y. Kondr. \& Kärnefelt - Al: Colmeiro (1867), Egea \& Llimona (1981a, 1983, 1987), Silvestre \& Rowe (1982), Llimona \& Egea (1985); Ca: Colmeiro (1867); Gr: Colmeiro (1867), Sampaio (1917), Degelius (1966), Casares \& Llimona (1982, 1989), Egea et al. (1982), Prieto \& Espinosa (1971), Pereira et al. (1987), J: Aragón \& Rico (1997). Las citas próximas al mar corresponden a $X$. resendei.

Sagiolechia protuberans (Ach.) A. Massal. - Gr: Casares \& Llimona $(1983,1989)$

Sarcogyne clavus (DC.) Kremp. - Ca: Rowe \& Egea (1988); Gr: Egea et al. (1982); H: Rowe \& Egea (1987a); Se: Rowe \& Egea (1985b).

Sarcogyne privigna (Ach.) A. Massal. - Al: Egea \& Llimona (1981a, b); Gr: Casares \& Llimona (1989).

Sarcogyne privigna var. calcicola H. Magn. -Al: Casares \& Gutiérrez (1993), Gutiérrez \& Casares (1994); Ca: Rowe et al. (1996).

Sarcogyne regularis Körb. - Al: Casares \& Llimona (1986), Alonso \& Egea (1996); Ca: Ariño et al. (1995), Ariño \& Saiz-Jimenez (1996), Alonso \& Egea (1996), Ariño et al. (1997); Gr: Casares \& Llimona (1989), Alonso \& Egea (1996).

Sarcogyne regularis var. intermedia (Körb.) N. S. Golubk. - Gr: Casares \& Llimona (1989).

Sarcogyne urceolata Anzi - Gr: Casares \& Llimona (1989).

\#Sarcopyrenia gibba Nyl. - Gr: Casares \& Llimona (1984, 1986), Navarro-Rosinés \& Hladun (1990).

Schismatomma hafellneri Egea \& Torrente - Ca: Torrente \& Egea (1989a).

Sclerophora pallida (Pers.) Y. J. Yao \& Spooner - J: Sarrión et al. (1999), Muñiz \& Hladun (2011).

Scoliciosporum gallurae Vězda \& Poelt - Ca: Rowe et al. (1996).

Scoliciosporum sarothamni (Vain.) Vězda - Ca: Fos (1998).

Scoliciosporum umbrinum (Ach.) Arnold - Al: Egea \& Llimona (1981a, b, 1983, 1987, 1994); Ca: Rowe et al. (1996); J: Aragón et al. (2006a).

Scoliciosporum umbrinum var. corticola (Anzi) Bagl. \& Carestía Ca: Rowe et al. (1996).

Seirophora contortuplicata (Ach.) Fröden - Gr: Casares \& Llimona (1983, 1989), Casares (1988); J: Aragón \& Rico (1997).

Seirophora lacunosa (P. Rupr.) Fröden - Al: Casares \& Gutiérrez (1993), Gutiérrez \& Casares (1994), Gaya \& Pérez (2002).

Seirophora villosa (Ach.) Fröden - Ca: Colmeiro (1867), Sampaio (1917); Gr: Colmeiro (1867).

Solenopsora candicans (Dicks.) J. Steiner - Al: Gutiérrez \& Casares (1994), Alonso \& Egea (1996); Ca: Rowe et al. (1996); Gr: Casares \& Llimona (1989). 
Solenopsora holophaea (Mont.) Samp. -Al: Egea \& Llimona (1981b, 1983, 1987, 1994), Gutiérrez \& Casares (1994); Ma: Seaward \& Arvidson (1997), Herrera et al. (2007).

Solenopsora olivacea (Dufour) H. Kilias - Gr: Casares \& Llimona (1989).

Solenopsora vulturiensis A. Massal. - Ma: Herrera et al. (2007).

Solitaria chrysophthalma (Degel.) Arup et al. - Ma: Rowe et al. (1996).

Solorina saccata (L.) Ach. - Ca: Colmeiro (1867); Gr: Colmeiro (1867), Casares \& Llimona (1983, 1989), Burgaz \& Martínez (2003); J: Aragón \& Rico (1997), Burgaz \& Martínez (2003); Ma: Colmeiro (1867), Burgaz \& Martínez (2003).

Solorina spongiosa (Ach.) Anzi - Gr: Casares (1988).

Sphaerophorus globosus (Huds.) Vain. - Ma: Martín-Osorio (1988).

Sphinctrina leucopoda Nyl. - Ca: Muñiz \& Hladun (2011).

Sphinctrina turbinata (Pers.) De Not. - Ca: Rowe \& Egea (1986).

Spilonema paradoxum Bornet - Ca: Rowe \& Egea (1988); Se: Llimona \& Egea (1985), Rowe \& Egea (1985a, b).

Sporastatia testudinea (Ach.) A. Massal. - Gr: Degelius (1966), Egea et al. (1982).

Squamarina cartilaginea (With.) P. James - Al: Colmeiro (1867), Casares \& Gutiérrez (1993), Gutiérrez \& Casares (1994), Martínez-Sánchez et al. (1994), Alonso \& Egea (1996); Ca: Colmeiro (1867), Abbayes (1945), Casares \& Rowe (1988), Rowe \& Egea (1988); *Co: MACB 103278; Gr: Colmeiro (1867), Sampaio (1917), Casares (1988), Casares \& Llimona (1989), Casares \& Gutiérrez (1993), Martínez-Sánchez et al. (1994), Alonso \& Egea (1996); H: Rowe et al. (1982); J: Aragón \& Rico (1997); Se: Barras (1896, 1899), Silvestre \& Rowe (1982), Rowe \& Egea (1985b).

Squamarina concrescens (Müll. Arg.) Poelt - Ca: Rowe et al. (1996); Gr: Poelt \& Krüger (1970), Casares \& Llimona (1989), Casares \& Gutiérrez (1993); Ma: Poelt \& Krüger (1970), Seaward \& Arvidson (1997); J: Poelt \& Krüger (1970); Se: Ariño et al. (1997).

Squamarina gypsacea (Sm.) Poelt - Al: Colmeiro (1867); Ca: Colmeiro (1867), Rowe et al. (1996); *Co: MACB 103281; Gr: P0elt \& Krüger (1970), Casares \& Llimona (1989); Ma: Colmeiro (1867), Poelt \& Krüger (1970).

Squamarina lentigera (Weber) Poelt -Al: Poelt \& Krüger (1970), Casares \& Gutiérrez (1993), Gutiérrez \& Casares (1994), MartínezSánchez et al. (1994), Alonso \& Egea (1996); Ca: Colmeiro (1867), Poelt \& Krüger (1970); Co: Colmeiro (1867); Gr: Colmeiro (1867), Poelt \& Krüger (1970), Casares \& Llimona (1989), Casares \& Gutiérrez (1993), Martínez-Sánchez et al. (1994), Alonso \& Egea (1996); H: Rowe et al. (1982); J: Colmeiro (1867), Aragón \& Rico (1997); Ma: Colmeiro (1867); Se: Barras (1896), Lázaro Ibiza (1898a), Barras (1899), Poelt \& Krüger (1970), Silvestre \& Rowe (1982)

Squamarina oleosa (Zahlbr.) Poelt - Ca: Rowe et al. (1996); *Co: MACB 103284; Gr: Casares \& Llimona (1989), Casares \& Gutiérrez (1993); H: Rowe et al. (1982).

Squamarina periculosa B. de Lesd. - Al: Alonso \& Egea (1996); Ca: Rowe et al. (1996); Gr: Poelt \& Krüger (1970), Casares \& Llimona (1989); Ma: Poelt \& Krüger (1970).

Squamarina stella-petraea Poelt - Gr: Poelt \& Krüger (1970).

Staurolemma omphalarioides (Anzi) P. M. Jørg. \& Henssen - Ca: Rowe et al. (1996), Fos (1998); *Co: MACB 103286; J: Aragón \& Rico (1997); Ma: Rowe et al. (1996).

Staurothele clopima (Wahlenb.) Th. Fr. - Gr: Casares \& Llimona $(1984,1986)$, Egea (1985).
Staurothele clopimoides (Bagl. \& Carestia) J. Steiner - Gr: Egea (1985b), Pereira et al. (1987).

Staurothele hazslinszkyi (Körb.) Blomb. \& Forssell -Al: Egea \& Llimona (1981a, 1987); Gr: Egea et al. (1982).

Staurothele hymenogonia (Nyl.) Th. Fr. - Ca: Rowe et al. (1996); Gr: Casares \& Llimona $(1984,1986)$, Alonso \& Egea (1996).

'Staurothele' immersa (A. Massal.) Dalla Torre \& Sarnth. -Al: Alonso \& Egea (1996).

Staurothele orbicularis (A. Massal.) Th. Fr. - Ca: Casares \& Rowe (1988), Rowe et al. (1996); Gr: Casares \& Llimona (1984).

Sticta canariensis (Ach.) Bory ex Delise - Ma: Seaward \& Arvidson (1997).

Sticta fuliginosa (Dicks.) Ach. - J: Merino \& Otálora (2011); Ma: Rowe et al. (1996).

Sticta limbata (Sm.) Ach. - Ma: Seaward \& Arvidson (1997), Fos (1998), Burgaz \& Martínez (2003).

\#Stigmidium hageniae (Rehm) Hafellner - Ma: Rowe et al. (1996).

\#Stigmidium pseudopeltideae Cl. Roux \& Triebel - J: Martínez \& Hafellner (1998), Martínez (1999).

\#Stigmidium schaereri (A. Massal.) Trevis. - Ma: Rowe et al. (1996).

Synalissa ramulosa (Bernh.) Körb. - Gr: Casares \& Llimona (1989).

Teloschistes chrysophthalmus (L.) Beltr. - Ca: Colmeiro (1867), Silvestre \& Rowe (1982), Rowe \& Egea $(1986,1988)$, Rowe et al. (1996), Fos (1998), Hernández Gallego et al. (1998); *Co: MACB 103355; Ma: Fos (1998).

Teloschistes flavicans (Sw.) Norman - Ca: Abbayes (1945), Rowe \& Egea (1987b), Rowe et al. (1996).

Tephromela atra (Huds.) Hafellner -Al: Colmeiro (1867), Egea \& Llimona (1981a, b, 1983, 1987, 1994); Ca: Colmeiro (1867), Rowe \& Egea (1986, 1988), Egea \& Rowe (1987), Barreno et al. (1988), Rowe et al. (1996); Gr: Colmeiro (1867), Casares \& Llimona (1989); J: Aragón et al. (2006a); Ma: Martín-Osorio (1988), Rowe et al. (1996).

Tetramelas pulverulentus (Anzi) A. Nordin \& Tibell - Ma: Rowe et al. (1996).

Thelenella modesta (Nyl.) Nyl. - Ca: Fos (1998), Etayo \& Mayrhoffer (2003).

Thelenella muscorum (Fr.) Vain. - Ma: Rowe et al. (1996).

Thelidium decipiens (Hepp) Kremp. - Ca: Casares \& Rowe (1988); Gr: Casares \& Llimona (1984).

Thelidium impressulum Zschacke - Ca: Casares \& Rowe (1988).

Thelopsis isiaca Stizenb. -Al: Alonso \& Egea (1996); Ma: Herrera et al. (2007).

Thyrea confusa Henssen - Ca: Moreno \& Egea (1992).

Thyrea girardii (Durieu \& Mont.) Bagl. \& Carestia - Ca: Moreno \& Egea (1992); Gr: Casares \& Llimona (1983, 1989), Moreno \& Egea (1992).

Thyrea nummularia (Nyl.) Zahlbr. - Gr: Casares \& Llimona (1989).

Thyrea plicatissima (Nyl.) Zahlbr. -Al: Moreno \& Egea (1989, 1992).

Toninia albilabra (Dufour) H. Olivier - Al: Timdal (1991), Al: Casares \& Gutiérrez (1993), Gutiérrez \& Casares (1994); Gr: Colmeiro (1867), Timdal (1991).

Toninia aromatica (Turner) A. Massal. - Al: Egea \& Llimona (1981b, 1983, 1987, 1994), Casares \& Gutiérrez (1993), Alonso \& Egea (1996); Ca: Ariño et al. (1995, 1997), Alonso \& Egea (1996); Gr: Casares \& Llimona (1986, 1989), Timdal (1991), Alonso \& Egea (1996); Ma: Rowe et al. (1996); Se: Ariño et al. (1997).

Toninia candida (Weber) Th. Fr. - Al: Colmeiro (1867), Alonso \& Egea (1996); Ca: Colmeiro (1867); *Co: MACB 103357; Gr: Timdal (1991), Casares (1988), Casares \& Llimona $(1986,1989)$; 
J: Colmeiro (1867), Aragón \& Rico (1997); Ma: Colmeiro (1867).

Toninia caulescens Anzi - Gr: Egea et al. (1982).

Toninia diffracta (A. Massal.) Zahlbr. - Ca: Casares \& Rowe (1988); Gr: Timdal (1991), Casares (1988); Casares \& Llimona (1989), J: Aragón \& Rico (1997).

Toninia episema (Nyl.) Timdal - Gr: Casares (1988).

Toninia lobulata (Sommerf.) Lynge - Gr: Casares (1988), Casares \& Llimona (1989).

Toninia lutosa (Ach.) Timdal -Al: Timdal (1991), Gutiérrez \& Casares (1994).

Toninia opuntioides (Vill.) Timdal -Al: Timdal (1991), Alonso \& Egea (1996); Ca: Rowe et al. (1996); Gr: Timdal (1991), Casares (1988), Casares \& Llimona (1989); J: Aragón \& Rico (1997).

Toninia philippea (Mont.) Timdal - Gr: Timdal (1991).

Toninia plumbina (Anzi) Hafellner \& Timbal - Ma: Rowe et al. (1996).

Toninia ruginosa (Tuck.) Herre-Al: Timdal (1991); Gr: Timdal (1991).

Toninia sbarbaronis B. de Lesd. -Al: Egea \& Llimona (1981a, b, 1983).

Toninia sedifolia (Scop.) Timdal-Al: Abbayes (1945), Llimona (1974), Timdal (1991), Casares \& Gutiérrez (1993), Gutiérrez \& Casares (1994), Martínez-Sánchez et al. (1994), Alonso \& Egea (1996); Ca: Timdal (1991), Rowe et al. (1996); *Co: MACB 103359; Gr: Colmeiro (1867), Sampaio (1917), Casares (1988), Casares \& Llimona (1989), Timdal (1991), Casares \& Gutiérrez (1993), Martínez-Sánchez et al. (1994), Alonso \& Egea (1996); H: Rowe et al. (1982); J: Aragón \& Rico (1997); Ma: Colmeiro (1867); Se: Colmeiro (1867).

Toninia squalida (Ach.) A. Massal. - Al: Timdal (1991), Egea \& Llimona (1981a, 1983, 1985), Alonso \& Egea (1996); Gr: Timdal (1991), Egea et al. (1982), Casares \& Llimona (1989); Ma: Herrera et al. (2007); Se: Rowe \& Egea (1985a, b).

Toninia taurica (Szatala) Oxner - Gr: Timdal (1991).

Toninia toepfferi (Stein) Navás - Gr: Timdal (1991), Alonso \& Egea (1996); Ma: Timdal (1991).

Toninia tristis (Th. Fr.) Th. Fr. - Gr: Casares \& Llimona $(1986,1989)$.

Toninia tristis subsp. pseudotabacina Timdal-Al: Timdal (1991); Gr: Timdal (1991).

Toninia tumidula (Sm.) Zahlbr. -Al: Egea \& Llimona (1981b), Alonso \& Egea (1996); Ca: Casares \& Rowe (1988), Rowe et al. (1996); *Co: MACB 103360; Gr: Casares \& Llimona (1989).

Toninia verrucarioides (Nyl.) Timdal - Ca: Rowe et al. (1996); Gr: Casares \& Llimona (1989).

Topelia heterospora (Zahlbr.) P. M. Jørg. \& Vězda - Gr: Casares \& Llimona (1984), Alonso \& Egea (1996).

\#Tremella macrobasidiata J. C. Zamora et al. - J: Zamora et al. (2011).

** Trapelia coarctata (Sm.) M. Choisy - Co: MACB 103362; Ma: MACB 103364; Se: MACB 103363.

Trapeliopsis flexuosa (Fr.) Coppins \& P. James - Ca: Fos (1998); H: Fos (1998).

Trapeliopsis gelatinosa (Flörke) Coppins \& P. James - J: Aragón et al. (2006a).

Trapeliopsis granulosa (Hoffm.) Lumbsch-Al: Colmeiro (1867); Ca: Colmeiro (1867), Fos (1998); Gr: Colmeiro (1867); J: Aragón et al. (2006a); Ma: Colmeiro (1867).

Tremolecia atrata (Ach.) Hertel - Ca: Rowe \& Egea (1987b), Rowe et al. (1996).

Tuckermanopsis chlorophylla (Willd.) Hale - J: Aragón \& Rico (1997); Aragón et al. (2006a); Ma: Seaward \& Arvidson (1997).

Umbilicaria cinerascens (Nyl.) Nyl. - Gr: Egea et al. (1982).
Umbilicaria cinereorufescens (Schaer.) Frey - Gr: Casares \& Llimona (1982).

Umbilicaria crassa Colmeiro - Gr: Colmeiro (1867).

Umbilicaria crustulosa (Ach.) Lamy - Ca: Egea \& Rowe (1987), Rowe \& Egea (1988); Gr: Sampaio (1917).

Umbilicaria cylindrica (L.) Delise - Gr: Werner (1979), Egea et al. (1982).

Umbilicaria decussata (Vill.) Zahlbr. - Al: Egea \& Llimona (1981a, 1987); Gr: Degelius (1966), Prieto \& Espinosa (1971), Casares \& Llimona (1982), Egea et al. (1982).

Umbilicaria depressa (Ach.) Duby - Gr: Casares \& Llimona (1982).

Umbilicaria grisea Hoffm. - Al: Egea \& Llimona (1981a, 1987); Ca: Rowe \& Egea (1986, 1988), Egea \& Rowe (1987); *Co: MACB 103163; Gr: Colmeiro (1867), Egea et al. (1982); H: Rowe \& Egea (1987a); Se: Rowe \& Egea (1987a).

Umbilicaria hirsuta (Sw. ex Westr.) Hoffm. - Gr: Colmeiro (1867), Prieto \& Espinosa (1971).

Umbilicaria leiocarpa DC. - Ca: Colmeiro (1867).

Umbilicaria nylanderiana (Zahlbr.) H. Magn. - Al: Egea \& Llimona (1981a); Gr: Casares \& Llimona (1982), Egea et al. (1982).

Umbilicaria polyphylla (L.) Baumg. - Ca: Colmeiro (1867), Rowe \& Egea (1987b), Rowe et al. (1996).

Umbilicaria proboscidea (L.) Schrad. - Gr: Colmeiro (1867).

Umbilicaria subglabra (Nyl.) Harm. - Gr: Prieto \& Espinosa (1971).

Umbilicaria vellea (L.) Ach. -Al: Egea \& Llimona (1981a, 1987); Ca: Colmeiro (1867); Gr: Colmeiro (1867), Egea et al. (1982).

Umbilicaria virginis Schrad. - Gr: Degelius (1966), Casares \& Llimona (1982), Egea et al. (1982).

Umbilicaria virginis var. meylani Frey - Gr: Casares \& Llimona (1982).

\#Unguiculariopsis lettaui (Grummann) Coppins - Ca: Rowe et al. (1996), Diederich \& Etayo (2000); Co: Diederich \& Etayo (2000); Ma: Rowe et al. (1996).

Usnea articulata (L.) Hoffm. - Ca: Rowe \& Egea (1986).

Usnea ceratina Ach. - Ca: Rowe \& Egea (1986), Fos (1998), Fos \& Clerc (2000); Ma: Rowe et al. (1996), Fos (1998); Se: Barras (1896).

Usnea comosa subsp. praetervisa Asahina-Ma: Seaward \& Arvidson (1997).

Usnea cornuta Körb. - H: Rowe et al. (1982); Ma: Seaward \& Arvidson (1997).

**Usnea diplotypus Vain. - Co: MACB 106494.

Usnea esperantiana P. Clerc - Ca: Fos (1998), Fos \& Clerc (2000).

Usnea filipendula Stirt. - Ma: Sampaio (1917), Seaward \& Arvidson (1997).

Usnea flammea Stirt. - Ca: Rowe et al. (1996); Ma: Rowe et al. (1996), Seaward \& Arvidson (1997).

Usnea fulvoreagens (Räsänen) Räsänen - *Co: MACB 106495; H: Rowe et al. (1982), Fos (1998), Fos \& Clerc (2000).

Usnea glabrata (Ach.) Vain. - Ca: Fos (1998); H: Fos (1998), Fos \& Clerc (2000); Ma: Seaward \& Arvidson (1997).

Usnea glabrescens (Nyl. ex Vain.) Vain. ex Räsänen - ${ }^{*}$ Co: MACB 106498.

Usnea hirta (L.) Weber ex F. H. Wigg. - Ca: Colmeiro (1867); H: Fos (1998), Fos \& Clerc (2000); J: Aragón \& Rico (1997), Aragón et al. (2006a).

Usnea lapponica Vain. - Ma: Seaward \& Arvidson (1997).

Usnea marocana Motyka - Ca: Barreno et al. (1988).

Usnea plicata (L.) Weber ex F. H. Wigg. - Ca: Colmeiro (1867); Gr: Colmeiro (1867); Ma: Colmeiro (1867).

Usnea rubicunda Stirt. - Ca: Abbayes (1945), Rowe \& Egea (1986), 
Barreno et al. (1988), Rowe et al. (1996), Fos (1998), Fos \& Clerc (2000); H: Fos (1998), Fos \& Clerc (2000).

Usnea subfloridana Stirt. - Ca: Fos \& Clerc (2000); H: Fos (1998), Fos \& Clerc (2000); J: Aragón et al. (2006a); Ma: Rowe et al. (1996), Seaward \& Arvidson (1997), Fos (1998).

Usnea subscabrosa Nyl. ex Motyka - Ca: Fos (1998), Fos \& Clerc (2000); H: Fos \& Clerc (2000).

Usnea substerilis Motyka - Gr: Fos (1998), Fos \& Clerc (2000).

Usnea wasmuthii Räsänen - Ca: Rowe et al. (1996), Fos (1998), Fos \& Clerc (2000); *Co: MACB 106496; H: Fos (1998), Fos \& Clerc (2000); J: Aragón et al. (2006a); Ma: Rowe et al. (1996), Seaward \& Arvidson (1997).

Usnochroma carphinea (Fr.) Søchting et al. - Al: Egea \& Llimona (1981a, 1983, 1987, 1994), Silvestre \& Rowe (1982); Ca: Rowe \& Egea (1988); *Co: MACB 103153; H: Crespo et al. (1976), Rowe et al. (1982), Rowe \& Egea (1987a); J: Sampaio (1917), Crespo et al. (1976), Egea (1984); Ma: Egea (1984), Seaward \& Arvidson (1997); Se: Egea (1984), Rowe \& Egea (1985a, b, 1987a).

Usnochroma scoriophila (A. Massal.) Søchting et al. - Al: Llimona (1975), Egea \& Llimona (1983, 1994); *Co: MACB 103154; Ma: Herrera et al. (2007).

Vahliella saubinetii (Mont.) P. M. Jørg. - J: Aragón \& Rico (1997), Paz-Bermúdez et al. (2010b).

Variospora aurantia (Pers.) Arup et al. - Al: Egea (1984), Alonso \& Egea (1996), Egea \& Llimona (1997); Ca: Ariño et al. (1995), Rowe et al. (1996); *Co: MACB 103319; Gr: Egea (1984), Casares \& Llimona (1986, 1989), Alonso \& Egea (1996); Se: Barras (1896, 1899), Ariño et al. (1997).

Variospora flavescens (Huds.) Arup et al. - Al: Egea (1984), Egea \& Llimona (1987, 1994), Gutiérrez \& Casares (1994), Alonso \& Egea (1996); Ca: Casares \& Rowe (1988), Ariño et al. (1995, 1997); *Co: MACB 103323; Gr: Egea (1984), Casares \& Llimona (1986, 1989); Ma: Herrera et al. (2007); Se: Rowe \& Egea (1987a), Ariño et al. (1997).

Verrucaria aethioloba Wahlenb. - H: Valcárcel \& López de Silanes (2010).

Verrucaria apomelaena Hepp - Gr: Prieto \& Espinosa (1971).

Verrucaria aquatilis Mudd - Gr: Pereira et al. (1987); J: Valcárcel \& López de Silanes (2010).

Verrucaria cinereorufa E. A. Schaer. - Ca: Rowe et al. (1996).

Verrucaria collematodes Garov. - Ca: Fos (1998).

Verrucaria compacta (A. Massal.) Jatta - Al: Prieto et al. (2010).

Verrucaria elaeomelaena (A. Massal.) Arnold - J: Valcárcel \& López de Silanes (2010).

Verrucaria funckii (Spreng.) Zahlbr. - H: Valcárcel \& López de Silanes (2010).

Verrucaria glaucina Ach. - Al: Alonso \& Egea (1996); Ca: Casares \& Rowe (1988); Gr: Casares \& Llimona $(1984,1986)$, Egea (1985), Alonso \& Egea (1996).

Verrucaria helveticorum Zehetl. - Ca: Ariño et al. (1995).

'Verrucaria' hochstetteri Fr. - Al: Alonso \& Egea (1996); Ca: Ariño et al. (1997); Gr: Casares \& Llimona (1984).

Verrucaria hydrela Ach. - Gr: Pereira et al. (1987); Se: Ariño et al. (1997).

'Verrucaria' macrostoma Dufour ex DC. -Al: Egea \& Llimona (1981b, 1994), Alonso \& Egea (1996); Ca: Ariño et al. (1995, 1997); Se: Ariño et al. (1997).

Verrucaria margacea (Wahlenb.) Wahlenb. - Gr: Egea et al. (1982), Pereira et al. (1987).

Verrucaria muralis Ach. - Al: Alonso \& Egea (1996); Ca: Colmeiro
(1867), Ariño et al. (1995), Ariño \& Saiz-Jimenez (1996), Rowe et al. (1996); Ariño et al. (1997); Gr: Colmeiro (1867), Sampaio (1917), Egea (1985); Se: Barras (1896, 1899), Silvestre \& Rowe (1982).

Verrucaria murorum (Arnold) Lindau - Al: Alonso \& Egea (1996); Ca: Rowe et al. (1996); Gr: Casares \& Llimona (1984).

'Verrucaria'nigrescens Pers. -Al: Egea \& Llimona (1994), Alonso \& Egea (1996); Ca: Colmeiro (1867), Rowe \& Egea (1986, 1988), Casares \& Rowe (1988), Alonso \& Egea (1996), Rowe et al. (1996), Ariño et al. (1997); *Co: MACB 103895; Gr: Colmeiro (1867), Casares \& Llimona (1984, 1986), Casares (1988), Alonso \& Egea (1996); Ma: Herrera et al. (2007); Se: Puertas et al. (1994), Ariño et al. (1997).

Verrucaria obductilis (Nyl.) Zschacke - Al: Gutiérrez \& Casares (1994).

Verrucaria pinguicula A. Massal. - Ca: Rowe et al. (1996); Gr: Casares \& Llimona (1984).

Verrucaria sorbinea Breuss - J: Aragón \& Sarrión (2003).

Verrucaria sphaerospora Anzi - Al: Egea \& Llimona (1981a, 1987); Ca: Rowe et al. (1996); Gr: Egea et al. (1982), Casares \& Llimona $(1984,1986)$.

'Verrucaria' viridula (Schrad.) Ach. - Ca: Ariño et al. (1997); Se: Ariño et al. (1997).

Verruculopsis lecideoides (A. Massal.) Gueidan \& Cl. Roux -Al: Egea \& Llimona (1981a, 1987, 1994), Alonso \& Egea (1996); Ca: Casares \& Rowe (1988), Rowe et al. (1996); *Co: MACB 103893; Gr: Casares \& Llimona (1984, 1986), Casares (1988).

Waynea adscendens V. J. Rico - Ca: Rowe et al. (1996); *H: MACB 106392; J: Aragón \& Sarrión (2003), Llop (2006); Ma: Rowe et al. (1996).

\#Wernerella maheui (Werner) Nav.-Ros. et al. - Ma: Navarro-Rosinés et al. (1998).

Xalocoa ocellata (Vill.) Kraichak et al. - Al: Alonso \& Egea (1996), Martín et al. (2000); Ca: Colmeiro (1867); *Co: MACB 103159; Gr: Colmeiro (1867), Sampaio (1917), Casares \& Llimona (1989), Alonso \& Egea (1996), Winka et al. (1998); Se: Barras (1896, 1899), Silvestre \& Rowe (1982); Ma: Colmeiro (1867), Lumbsch (1993).

Xanthocarpia ferrari (Bagl.) Fröden et al. - Gr: Egea (1984).

Xanthocarpia lactea (A. Massal.) A. Massal. -Al: Egea (1984), Egea \& Llimona (1994), Alonso \& Egea (1996); Ca: Ariño et al. (1995, 1997), Ariño \& Saiz-Jimenez (1996), Rowe et al. (1996); Gr: Egea (1984), Casares \& Llimona (1986, 1989); Ma: Herrera et al. (2007); Se: Puertas et al. (1994), Ariño et al. (1997).

Xanthocarpia marmorata (Bagl.) Fröden et al. - Ca: Ariño et al. (1995).

Xanthocarpia ochracea (Schaer.) A. Massal. \& De Not. - Al: Alonso \& Egea (1996); Gr: Egea (1984), Casares \& Llimona (1986, 1989).

Xanthomendoza fallax (Hepp ex Arnold) Søchting et al. - Gr: Casares \& Llimona (1989).

Xanthoparmelia conspersa (Ehrh. ex Ach.) Hale -Al: Egea \& Llimona (1981a, 1983, 1987), Alonso \& Egea (1996); Ca: Barreno et al. (1988), Fos (1998); *Co: MACB 103304; Gr: Colmeiro (1867); H: Rowe et al. (1982); J: Sampaio (1917); Ma: Rowe et al. (1996); Se: Barras (1896, 1899), Navás (1901), Rowe \& Egea (1985b), Rowe \& Egea (1987a).

Xanthoparmelia glabrans (Nyl.) O. Blanco et al. -Al: Egea \& Llimona (1981a, 1983, 1987).

Xanthoparmelia loxodes (Nyl.) O. Blanco et al. - Ca: Fos (1998); Ma: Rowe et al. (1996).

Xanthoparmelia pokornyi (Körb) O. Blanco et al. -Al: Casares \& Gu- 
tiérrez (1993), Gutiérrez \& Casares (1994), Martínez-Sánchez et al. (1994); Gr: Casares \& Gutiérrez (1993).

**Xanthoparmelia protomatrae (Gyeln.) Hale - Co: MACB 103306.

Xanthoparmelia pulla (Ach.) O. Blanco et al. - Al: Llimona (1975), Egea \& Llimona (1981a, b, 1987, 1994), Alonso \& Egea (1996) Souza-Egipsy et al. (2002); Ca: Rowe \& Egea $(1986,1988)$, Egea \& Rowe (1987), Rowe et al. (1996); *Co: MACB 103307; Gr: Werner (1979), Egea et al. (1982), Casares \& Llimona (1989), Alonso \& Egea (1996); H: Rowe et al. (1982), Rowe \& Egea (1987a); J: Sampaio (1917); Se: Barras (1896, 1899), Llimona \& Egea (1985), Rowe \& Egea (1985a, b, 1987a).

Xanthoparmelia stenophylla (Ach.) Ahti \& D. Hawksw. - Al: Egea \& Llimona (1983); Ca: Rowe et al. (1996), Fos (1998); *Co: MACB 103310; H: Rowe et al. (1982).

Xanthoparmelia tinctina (Maheu \& A. Gillet) Hale -Al: Egea \& Llimona (1981a, 1983, 1985, 1987, 1994), Casares \& Gutiérrez (1993), Gutiérrez \& Casares (1994); Ca: Rowe \& Egea (1986, 1988), Egea \& Rowe (1987), Rowe et al. (1996), Fos (1998); *Co: MACB 103312; H: Rowe \& Egea (1987a); J: Sarrión \& Burgaz (1995); Ma: Seaward \& Arvidson (1997); Se: Llimona \& Egea (1985), Rowe \& Egea (1985a, b, 1987a, b).

Xanthoparmelia verrucigera (Nyl.) Hale - Ca: Fos (1998).

Xanthoparmelia verruculifera (Nyl.) 0. Blanco et al. - Al: Egea \& Llimona (1981a, b, 1983), Egea \& Llimona (1987); Ca: Rowe \& Egea (1986), Rowe et al. (1996), Fos (1998); *Co: MACB 103315; H: Rowe \& Egea (1987a), Fos (1998); Se: Rowe \& Egea (1985b, 1987a).

Xanthoria aureola (Ach.) Erichsen -Al: Llimona (1975), Egea \& Llimona (1981a, 1987), Llimona \& Egea (1985); Ca: Rowe \& Egea (1986); Gr: Casares \& Llimona (1986), Casares (1988); H: Rowe \& Egea (1987a); Se: Llimona \& Egea (1985), Rowe \& Egea (1985a, b)

Xanthoria calcicola Oxner -Al: Casares \& Gutiérrez (1993), Egea \& Llimona (1994), Alonso \& Egea (1996), Egea \& Llimona (1997); Ca: Ariño et al. (1995), Alonso \& Egea (1996), Rowe et al. (1996); *Co: MACB 103316; Gr: Casares \& Llimona (1989), Alonso \& Egea (1996); J: Aragón \& Rico (1997); Ma: Herrera et al. (2007).

Xanthoria candelaria (L.) Th. Fr. - Al: Colmeiro (1867), Egea \& Llimona (1981a); Ca: Colmeiro (1867); Gr: Colmeiro (1867); J: Aragón et al. (2006a).

Xanthoria parietina (L.) Beltr. - Al: Egea \& Llimona (1981a, 1987), Souza-Egipsy et al. (2002); Ca: Colmeiro (1867), Barreno et al. (1988), Rowe et al. (1996), Fos (1998), Hernández Gallego et al. (1998); *Co: MACB 103317; Gr: Colmeiro (1867), Abbayes (1945), Werner (1979), Egea et al. (1982), Fos (1998), Honegger et al. (2004); H: Rowe et al. (1982); J: Aragón \& Rico (1997); Aragón et al. (2006a); Ma: Colmeiro (1867), Rowe et al. (1996), Fos (1998), Herrera et al. (2007); Se: Barras (1896, 1899), Lázaro Ibiza (1898b), Silvestre \& Rowe (1982).

Xanthoria resendei Poelt \& Tav. -Al: Llimona (1975), Egea \& Llimona $(1983,1994)$, Alonso \& Egea (1996); Gr: Alonso \& Egea (1996); Ma: Herrera et al. (2007).

Xyloschistes platytropa (Nyl.) Vain. - J: Aragón et al. (2004c).

Zamenhofia coralloidea (P. James) Clauzade \& Cl. Roux - Al: Gutiérrez \& Casares (1994); Ca: Fos (1998).

Especies excluidas o dudosas

Acarospora granatensis Samp. - Gr: Sampaio (1917). Probable A. nodulosa.

Acarospora schleicheri (Ach.) A. Massal. -Al: Colmeiro (1867); Co:
Colmeiro (1867); Gr: Colmeiro (1867); Ma: Colmeiro (1867). Identificaciones dudosas, por lo menos en parte, aunque las citas sobre suelo neutro pueden ser ciertas.

Acrocordia gemmata (Ach.) A. Massal. - Gr: Colmeiro (1867), Lázaro Ibiza (1898b).

Arthonia dispersa (Schrad.) Nyl. - Ca: Colmeiro (1867).

Arthonia varians (Davies) Nyl. - Gr: Colmeiro (1867).

Arthopyrenia punctiformis (Schrank) A. Massal. - Ma: Colmeiro (1867).

Biatora sphaeroides (Dicks.) Hornem. - Se: Lázaro Ibiza (1898a).

Biatora vernalis (L.) Fr. - Ca: Colmeiro (1867).

Cladonia deformis (L.) Hoffm. - Gr: Colmeiro (1867).

Cladonia digitata (L.) Hoffm. - Ca: Colmeiro (1867).

Cladonia rangiferina (L.) Weber ex F. H. Wigg. -Al: Colmeiro (1867); Ca: Colmeiro (1867); Gr: Colmeiro (1867); Se: Colmeiro (1867), Lázaro Ibiza (1898b).

Cladonia uncialis (L.) Weber ex F. H. Wigg. - Se: Lázaro Ibiza (1898b).

Collema gaditanum Clemente in Colmeiro - Ca: Colmeiro (1867); Ma: Colmeiro (1867).

Dibaeis baeomyces (L. f.) Rambold \& Hertel - Se: Lázaro Ibiza (1898b).

Diploschistes scruposus var. violarius (Nyl.) Lettau -Al: Egea \& Llimona (1981a, 1983).

Endocarpon atrovirens Clemente - Gr: Colmeiro (1867).

Flavocetraria nivalis (L.) Kärnefelt \& A. Thell - Gr: Colmeiro (1867).

Fuscidea kochiana (Hepp) V. Wirth \& Vězda - Gr: Colmeiro (1867).

Gomphillus calycioides (Delise ex Duby) Nyl. - Gr: Colmeiro (1867).

Graphis scripta (L.) Ach. - Ca: Colmeiro (1867); Gr: Colmeiro (1867); Se: Lázaro Ibiza (1898a).

Heterodermia speciosa (Wulfen) Trevis. - Ca: Colmeiro (1867).

Hydropunctaria maura (Wahlenb.) Keller et al. -Al: Colmeiro (1867); Ca: Colmeiro (1867). Es más verosimil la cita de Cádiz.

Lecanora allophana (Ach.) Nyl. - Ca: Colmeiro (1867).

Lecanora atrynea (Ach.) Nyl. - Se: Barras (1896).

Lecanora cadubriae (A. Massal.) Hedl. - Ca: Colmeiro (1867).

Lecanora coryli Colmeiro - Gr: Colmeiro (1867).

Lecanora farinaria Borrer - Ca: Colmeiro (1867).

Lecanora mosaica (Clemente) Colmeiro - Ma: Colmeiro (1867).

Lecanora pulchella Colmeiro - Al: Colmeiro (1867).

Lecanora rupicola var. sulphurata (Ach.) Clauzade \& Cl. Roux -Al: Colmeiro (1867); Ca: Colmeiro (1867).

Lecanora subfusca (L.) Ach. - Al: Colmeiro (1867); Ca: Colmeiro (1867); Gr: Colmeiro (1867); Se: Barras (1896), Lázaro Ibiza (1898a), Barras (1899), Silvestre \& Rowe (1982).

Lecidea albocoerulescens (Wulfen) Ach. - Al: Egea \& Llimona (1981a). Probablemente Porpidia albocerulescens.

Lecidea cana Clemente - Gr: Colmeiro (1867).

Lecidea cisticola Clemente - Ca: Colmeiro (1867).

Lecidea deformis Clemente - Gr: Colmeiro (1867).

Lecidea lapicida (Ach.) Ach. - Ca: Colmeiro (1867); Gr: Colmeiro (1867).

Lecidea pellucida Clemente - Ca: Colmeiro (1867).

Lecidea plicata Clemente - Ca: Colmeiro (1867); Co: Lázaro Ibiza (1898a).

Lecidea silacea (Hoffm.) Ach. - Ca: Colmeiro (1867).

Lecidea spongiosa Clemente - Ma: Colmeiro (1867).

Lecidea vesicularis (Hoffm.) Ach. - Se: Barras (1896).

Lempholemma hispanicum (Samp.) Zahlbr. - Ma: Sampaio (1917).

Letharia vulpina (L.) Hue - Ca: Colmeiro (1867); Gr: Colmeiro (1867); Ma: Colmeiro (1867).

Melanohalea olivacea (L.) O. Blanco et al. -Al: Colmeiro (1867); Ca: 
Colmeiro (1867), Hernández Gallego et al. (1998); Co: Colmeiro (1867); Gr: Colmeiro (1867); Ma: Colmeiro (1867); Se: Lázaro Ibiza (1898b).

Nephroma bellum (Spreng.) Tuck. - Ca: Rowe et al. (1996).

Pannaria ekistophylla Colmeiro - Gr: Colmeiro (1867).

Parmelia coccinea Clemente -Al: Colmeiro (1867).

Parmelia sirgii Clemente - Gr: Colmeiro (1867).

Peltigera aphthosa (L.) Willd. - Gr: Colmeiro (1867); J: Colmeiro (1867).

Peltigera degenii Gyeln. - Gr: Casares \& Llimona (1982). Cita errónea de P. rufescens.

Pertusaria subventosa var. subventosa Malme - Se: Barras (1896, 1899).

Phaeophyscia ciliata (Hoffm.) Moberg - Ca: Colmeiro (1867).

Placodium microcarpum Colmeiro - Gr: Colmeiro (1867).

Placodium variegatum Colmeiro - Gr: Colmeiro (1867).

Pleopsidium chlorophanum (Wahlenb.) Zopf - Al: Colmeiro (1867); Gr: Colmeiro (1867), Sampaio (1917); Ma: Colmeiro (1867).

Pseudephebe pubescens (L.) M. Choisy - Al: Colmeiro (1867); Ca: Colmeiro (1867); Gr: Colmeiro (1867).

Pseudocyphellaria aurata (Ach.) Vain. - Ca: Colmeiro (1867).

Ramalina evernioides Nyl. - Se: Barras (1896).

Roccella tinctoria DC. - Al: Colmeiro (1867); Ca: Colmeiro (1867); Ma: Colmeiro (1867).

Stereocaulon paschale (L.) Hoffm. - Se: Lázaro Ibiza (1898b).

Tornabea scutellifera (With.) J. R. Laundon - Al: Colmeiro (1867); Ca: Colmeiro (1867); Gr: Colmeiro (1867).
Umbilicaria atropapillata Colmeiro - Gr: Colmeiro (1867).

Usnea florida (L.) Weber ex F. H. Wigg. - Ca: Colmeiro (1867); Gr: Colmeiro (1867); Ma: Colmeiro (1867).

Vahliella leucophaea (Vahl) P. M. Jørg. - Gr: Colmeiro (1867).

Verrucaria acrotella Ach. - Ca: Colmeiro (1867).

Verrucaria baetica Clemente - Ca: Colmeiro (1867).

Verrucaria exigua Clemente - Gr: Colmeiro (1867).

Verrucaria fuscella (Turner) Winch - Ma: Colmeiro (1867).

Verrucaria gaditana Clemente - Ca: Colmeiro (1867).

Verrucaria incrustata Clemente - Ca: Colmeiro (1867); Gr: Colmeiro (1867).

Verrucaria prominula Nyl. - Gr: Colmeiro (1867).

Verrucaria suberis Clemente - Ca: Colmeiro (1867).

Verrucaria variegata Clemente - Ca: Colmeiro (1867).

Vulpicida juniperinus (L.) J.-E. Mattsson \& M. J. Lai - Gr: Colmeiro (1867).

\section{AGRADECIMIENTOS}

A Ignacio Jorge, Iván Melero y Francisco Miguelañez que colaboraron en los inicios del trabajo. A Xavier Llimona, por los acertados comentarios en el texto y la cuidadosa corrección. A Rosario Arroyo, por la confirmación de Ramalina subgeniculata, a Elena Araujo, por la identificación de Usnea diplotypus y la revisión de muestras del género Usnea.

\section{BIBLIOGRAFÍA}

ABBAYES, H. des.1945. Lichens d'Espagne récoltés de 1926 a 1935 par M. et Mme. Allorge. Rev. Bryol. Lichénol. 15(12): $79-86$.

Alonso, F. L. \& EgEA, J. M. 1996. Contribución a los líquenes calcícolas y terrícolas de algunas localidades costeras del sur de España. Anales de Biología (Biología Vegetal) 21: 55-72.

Aragón, G. 2003. Sobre la presencia de Catapyrenium daedaleum (Kremp.) Stein (Verrucariaceae, Lichenes) en España. Anales Jard. Bot. Madrid 60: 216-217.

ARAGón, G. \& MARTínez, I 1995. Cartografía corológica de líquenes ibéricos, II. Bot. Complut. 20: 117-137.

Aragón, G. \& Martínez, I. 2003. Bacidia iberica, a new lichen from Spain, and its relationship to Bacidia rubella. Bryologist 106(1): 143-146.

Aragón, G.; Martínez, I. \& Belinchón, R. 2006a. Aportación al conocimiento de los líquenes epífitos de Pinus halepensis, P. nigra, P. pinaster y $P$. sylvestris en la Península Ibérica. Bot. Complut. 30: 61-70.

Aragón, G.; Martínez, I. \& OtÁLora, M. A. G. 2004a. The lichen Leptogium subaridum, a new Mediterranean-NW American disjunction. Lichenologist 36: 163-165.

Aragón G.; MartíneZ, I. \& OtÁlora, M. A. G. 2004b. New data on the distribution of Leptogium azureum (Swartz) Mont. Lichenologist 36: 345-347.

Aragón, G. \& Rico, V. J. 1997. Los macrolíquenes del macizo del Calar del Mundo (Albacete) y de la Sierra de Segura (Jaén, España). Lazaroa 18: 45-93.
ARAgón, G.; Rico, V. J. \& Belinchón, R. 2006b. Lichen diversity from Cazorla, Segura and Las Villas Biosphere Reserve (SE, Spain). Nova Hedwigia 82(1-2): 31-50.

Aragón, G. \& Sarrión, F. J. 2003. Epiphytic Verrucariaceae (lichens) in Mediterranean Spain. Nova Hedwigia 77: 169-187.

Aragón G.; Sarrión, F. J. \& MarTínez, I. 2004c. Epiphytic lichens on Juniperus oxycedrus L. in the Iberian Peninsula. Nova Hedwigia 78(1-2): 45-56.

Ariño, X.; Ortega-Calvo, J. J.; Gomez-Bolea, A. \& Saiz-JIMENEZ, C. 1995. Lichen colonization of the Roman pavement at Baelo Claudia (Cadiz, Spain): biodeterioration vs. bioprotection. Sci. Total Environ. 167: 353-363.

ARIÑo, X. \& SAIZ-JiMENEZ, C. 1996. Lichen deterioration of consolidants used in the conservation of stone monuments. $\mathrm{Li}$ chenologist 28(4): 391-394.

Arroyo, R. 1991. El género Ramalina Ach. en la Península Ibérica: química, quimiotaxonomía, morfología, anatomía y distribución. Tesis Doctoral, UCM. Madrid.

Arroyo, R.; SeriÑÁ, E. \& Manrique, E. 1991. Estudio químico y morfológico de Ramalina polymorpha grex en España. Acta Bot. Malacitana 16(1): 165-174.

Arup, U.; SøChting, U. \& FrödÉN, P. 2013. A new taxonomy of the family Teloschistaceae. Nordic J. Bot. 31: 16-83.

Asso, I. J. 1779. Sinopsis stirpium indigenarum Aragoniae. Marsella.

Barbero, M.; Giralt, M.; Elix, J. A.; Gómez-Bolea, A. \& LliMONA, X. 2006. A taxonomic study of Protoparmelia mon- 
tagnei (syn. P. psarophana) centered in the Eastern Iberian Peninsula. Mycotaxon 97: 299-320.

BARRAS, F. de las 1896. Lista de líquenes de Andalucía que existen en el Museo de Historia Natural de la Universidad de Sevilla, coleccionados por D. Salvador Calderón. Actas Soc. Hist. Nat. marzo: 53-54.

Barras, F. de las 1899. Datos para la flórula sevillana. Actas Soc. Hist. Nat. febrero: 59-62.

Barrasa, J. M. \& Rico, V. J. 2003. The non-omphalinoid species of Arrhenia in the Iberian Peninsula. Mycologia 95(4): 700713.

Barreno, E.; Sanz, M. J.; Atienza, V. \& MuÑoz, A. 1988. Biogeografía y ecología comparadas de líquenes epífitos de alcornocales ibéricos y sardos. Actes Simposi Int. Bot. Pius Font $i$ Quer, I Criptogàmia: 179-185.

Boqueras, M. 1997. Els gèneres Ochrolechia i Pertusaria a l'herbari Werner. Acta Bot. Barc. 44:17-28.

Burgaz, A. R. 2009. El género Cladonia en la Península Ibérica. Supergrupo Cocciferae. Bot. Complut. 33: 9-28.

Burgaz, A. R. 2010. Nuevos datos sobre el género Placynthium en la Península Ibérica. Bot. Complut. 34: 5-19.

Burgaz, A. R. 2011a. Massalongiaceae y Placynthiaceae. Flora Liquenológica Ibérica, 8. Sociedad Española de Liquenología.

Burgaz, A. R. 2011b. Asientos de flora liquenológica ibérica: Massalongiaceae y Placynthiaceae. Clementeana 13: 16-50.

Burgaz, A. R. \& Ahti, T. 2009. Cladoniaceae. Flora Liquenológica Ibérica, 4. Sociedad Española de Liquenología.

Burgaz, A. R.; MarTínez, I. 2002. The Placynthium nigrum group in the Iberian Peninsula. Cryptogam. Mycol. 23: 367-375.

Burgaz, A. R. \& Martínez, I. 2003. Peltigerales: Lobariaceae, Nephromataceae, Peltigeraceae. Flora Liquenológica Ibérica, 1. Sociedad Española de Liquenología.

Calatayud, V.; Navarro-Rosinés, P. \& Calvo, E. 2000. Lichenochora mediterranea (Phyllacorales, Ascomycota), a new lichenicolous fungus from Spain. Lichenologist 32: 225 231.

Calatayud, V.; Navarro-Rosinés, P. \& Hafellner, J. 2002. A synopsis of Lichenostigma subgen. Lichenogramma (Arthoniales), with a key to the species. Mycol. Res. 106(10): 1230-1242.

Carballal, R. \& López de Silanes, M. E. 2010. Protopannaria y Psoroma. Flora Liquenológica Ibérica, 6. Sociedad Española de Liquenología.

Carballal, R.; Paz-Bermúdez, G. \& López de Silanes, M. E. 2010a. Pannaria. Flora Liquenológica Ibérica, 6. Sociedad Española de Liquenología.

Carballal, R.; Paz-Bermúdez, G. \& Pérez Valcárcel, C. 2010b. Degelia y Erioderma. Flora Liquenológica Ibérica, 6. Sociedad Española de Liquenología.

Carvalho, P. 2012. Collema. Flora Liquenológica Ibérica, 10. Sociedad Española de Liquenología.

CASARES, M. 1986. Schedae exsiccatae permutatione lichenologica (I). Folia Bot. Misc. 5: 79-84.

CAsares, M. 1988. Aspicilietum verrucosae Frey 1927 y Teloschistetum contortuplicati Asta et Roux 1977 en las calizas béticas de la provincia de Granada. Acta Bot. Malacitana 13: $111-120$

CASARES, M. 1992. Schedae exsiccatae permutatione lichenologica a Societatis Lichenologica Hispanica distributa. Fasciculum II. Folia Bot. Misc. 8: 45-50.

Casares, M. \& Gutierrez, L. 1993. Síntesis de la vegetación liquénica gipsícola termo - y mesomediterránea de la Península Ibérica. Cryptogam. Bryol. Lichénol. 14(4): 361388.

Casares, M., Hafellner, J. \& Gutierrez, L. 1996. Species of the genus Lecidea (Lecanorales) on gypsum in Spain. $\mathrm{Li}$ chenologist 28: 37-47.

Casares, M. \& Llimona, X. 1982. Líquenes de los peñones de San Francisco (Sierra Nevada, sur de España). Acta Bot. Malacitana 7: 5-10.

Casares, M. \& Llimona, X. 1983. Aportacion al conocimiento de los líquenes calcícolas de la provincia de Granada. Collect. Bot. (Barcelona) 14: 221-230.

Casares, M. \& Llimona, X. 1984. Algunos datos sobre los pirenolíquenes calcícolas de la provincia de Granada. Anales de Biología, 1 secc. especial 1: 207-218

Casares, M. \& Llimona, X. 1986. La clase Verrucarietea nigrescentis Wirth 1980 en las calizas béticas de la provincia de Granada. Cryptogam. Bryol. Lichenol. 7: 103-127.

Casares, M. \& Llimona, X. 1989. Catálogo de los líquenes calcícolas de la provincia de Granada (Andalucía, España). Discolíquenes. Acta Bot. Malacitana 14: 41-57.

Casares, M. \& Rowe, J. G. 1988. Algunos líquenes calcícolas del Parque Natural de Grazalema. Acta Bot. Malacitana 13: 290-296.

Clemente, S. DE R. 1807. Ensayo sobre las variedades de la vid común que vegetan en Andalucía, con un índice etimológico y tres listas de plantas en que se caracterizan varias especies nuevas. Madrid.

Clemente, S. DE R. 1863. Tentativa sobre la liquenología geográfica de Andalucía (Ed. M. Colmeiro). Rev. Progr. Ci. Exact. 14(2): 39-58.

Colmeiro, M. (1867) 1868. Enumeración de las criptógamas de España y Portugal. Parte segunda. Rev. Progr. Ci. Exact. 1718: $1-260$.

CResPí, L. 1930. Notas liquenológicas. El género Rhizocarpon en España. Bol. Real Soc. Esp. Hist. Nat. 30: 261-269.

Crespo, A.; Barreno, E. \& Follmann, G. 1976. Sobre las comunidades líquénicas rupícolas de Acarospora hilaris (Duf.) Hue en la Península Ibérica. Anales Inst. Bot. Cavanilles 33: 189-205

Crespo, A.; Blanco, O.; Llimona, X.; Ferencová, Z. \& HaWksWORTH, D. L. 2004. Coscinocladium gaditanum and overlooked endemic and monotypic Mediterranean lichen genus of Physciaceae, reinstated by molecular phylogenetic analysis. Taxon 53(2): 405-414.

Crespo, A. \& Bueno, A. G. 1984. Flora liquénica epifítica de Cádiz. I. Los alcornocales de las Sierras de Algeciras. Anales de Biología, 1 (Sección especial, 1): 219-231.

Crespo, A.; Divakar, P. K.; Argüello, A.; Gasca, C. \& HawksWORTH, D. L. 2004. Molecular studies on Punctelia species 
of the Iberian Peninsula, with an emphasis on specimens newly colonizing Madrid. Lichenologist 36: 299-308.

Degelius, G. 1954. The lichen genus Collema in Europe. Symbolae Bot. Upsal. 13(2): 1-499.

Degelius, G. 1966. Lichens of the summit of Picacho de Veleta (Sierra Nevada, Spain). A contribution to the knowledge of the flora of high altitudes. Svensk Bot. Tidskr. 60: 338-340.

Diederich, P. \& EtaYo, J. 2000. A synopsis of the genera Skyttea, Llimoniella and Rhymbocarpus (lichenicolous Ascomycota, Leotiales). Lichenologist 32: 423-485.

Divakar, P. K.; Blanco, O.; Hawksworth, D. L. \& Crespo, A. 2005. Molecular phylogenetic studies on the Parmotrema reticulatum (syn. Rimelia reticulata) complex, including the confirmation of $P$. pseudoreticulatum as a distinct species. Lichenologist 37: 55-65.

Divakar, P. K.; Del-Prado, R.; Lumbsch, H. T.; Wedin, M.; Esslinger, T. L.; LeavitT, S. D. \& Crespo, A. 2012. Diversification of the newly recognized lichen-forming fungal lineage Montanelia (Parmeliaceae, Ascomycota) and its relation to key geological and climatic events. Am. J. Bot. 99 (12): 2014-2026

EGEA, J. M. 1984. Contribución al conocimiento del género $\mathrm{Ca}$ loplaca Th. Fr. en España: especies saxícolas. Collect. Bot. (Barcelona) 15: 173-204.

EGEA, J. M. 1985. Algunos líquenes silicícolas interesantes de la Sierra de Filabres (Almería) y Sierra Nevada (Granada), España. Acta Bot. Malacitana 10: 3-10

EgeA, J. M. 1989. Los generos Heppia y Peltula (líquenes) en Europa Occidental y Norte de África. Biblioth. Lichenol. 31: 1-122.

Egea, J. M. \& Llimona, X. 1981a. Líquenes silicícolas de la sierra de los Filabres y sierra Alhamilla (Almería, España). Anales Univ. Murcia, Ciencias 37(1-4): 107-152

EGEA, J. M. \& Llimona, X. 1981b. Líquenes de rocas silíceas no volcánicas de localidades de escasa altitud del SE de España. Anales Univ. Murcia, Ciencias 37(1-4): 153-182.

EgeA, J. M. \& Llimona, X. 1983. Mapas de distribución en el S.E. de España de los principales líquenes silicícolas. I Anales Univ. Murcia, Ciencias 41(1-4): 209-252.

EgeA, J. M. \& Llimona, X. 1984. Las comunidades liquénicas saxícolas ombrófobas de la costa SE de España comprendidas entre Penyal d'Ifac (Alicante) y Almería. Collect. Bot. (Barcelona) 14: 265-269.

EgEA, J. M. \& Llimona, X. 1987. Las comunidades de líquenes de las rocas silíceas no volcánicas del SE de España. Acta Bot. Barcinonensia 36: 1-123.

EgEA, J. M. \& Llimona, X. 1994. La flore et la végétation lichéniques des laves acides du parc naturel de la Sierra del Cabo de Gata (SE de l'Espagne) et des régions voisines. Bull. Soc. Linn. Provence 45: 263-281.

Egea, J. M. \& Llimona, X. 1997. Sobre la flora y vegetación liquénicas de las lavas básicas del sureste de España. Acta Bot. Malacitana 22: 5-11.

Egea, J. M.; Llimona, X. \& Casares, M. 1982. Aportación al conocimiento de la flora liquénica silicícola de la parte cul- minal de Sierra Nevada. Collect. Bot. (Barcelona) 13(1): 295-312.

Egea, J. M.; Llimona, X. \& Manrique, E. 1995. Nuevos datos sobre Glyphopeltis ligustica (Psoraceae, Lecanorales) y su área de distribución. Flechten Follmann, University of $\mathrm{Co}$ logne, Cologne: 183-192.

Egea, J. M. \& Rowe, J. G. 1987. Pertusarietum mammoso-gallicae y Lecanactino-Dirinetum sorediatae, dos nuevas asociaciones liquénicas rupícolas. Anales Jard. Bot. Madrid 44(1): 119-130.

Egea, J. M. \& Torrente, P. 1985. Aportación al conocimiento de los líquenes calcícolas del litoral del S. E. de España. Int. J. Mycol. Lichenol. 2: 31-38.

EgeA, J. M. \& Torrente, P. 1993. The lichen genus Bactrospora. Lichenologist 25(3): 211-255.

Ertz, D.; LaWrey, J. D.; Common, R. S. \& Diederich, P. 2013. Molecular data resolve a new order of Arthoniomycetes sister to the primarily lichenized Arthoniales and composed of black yeasts, lichenicolous and rock-inhabiting species. Fungal Diversity 1-25.

Etayo, J. \& Mayrhoffer, H. 2003. Thelenella melanospora (Thelenellaceae, lichenized Ascomycetes), a new species from the Mediterranean region. Nova Hedwigia 77: 109114.

Follmann, G. 1973. Schedae ad Lichenes Exsiccati Selecti a Museo Historiae Naturalis Casselensi editi. V Fasiculus. Philippia 2: 13-21.

FollmanN, G. \& CRespo, A. 1974. Observaciones acerca de la distribución de líquenes españoles. II. Anales Inst. Bot. Cavanilles 31(1): 325-333.

Fos, S. 1998. Líquenes epífitos de los alcornocales ibéricos. Correlaciones bioclimáticas, anatómicas y densimétricas con el corcho de reproducción. Guineana 4: 1-507.

Fos, S. \& Clerc, P. 2000. The lichen genus Usnea on Quercus suber in Iberian cork-oak forests. Lichenologist 32(1): 67-88.

Fos, S.; Aragón, G. \& SARrión, F. J. 2000. Sobre la presencia de Rinodina dalmatica Zahlbr. en España. Cryptogam. Mycol. 21: 61-65.

Gaya, E. \& PÉrez, A. 2002. Primera citació de Teloschistes lacunosus (Rupr.) Savicz al País Valencià. But. Inst. Cat. Hist. Nat. 70: 48-51.

Giralt, M. 2010. Physciaceae I. Flora Liquenológica Ibérica, 5. Sociedad Española de Liquenología.

GiRaLt, M. \& Nordin, A. 2002. Buellia triseptata in the Iberian Peninsula. Lichenologist 34: 89-94.

Giralt, M. \& Barbero, M. 1995. The saxicolous species of the genus Rinodina in the Iberian Peninsula containing atranorin, pannarin or gyrophoric acid. Mycotaxon 56: 45-80.

Giralt, M. \& Llimona, X. 1997. The saxicolous species of the genera Rinodina and Rinodinella lacking spot test reactions in the Iberian Peninsula. Mycotaxon 62: 175-224.

Giralt, M. \& Mayrhofer, H. 1994. Four corticolous species of the genus Rinodina (lichenized Ascomycetes, Physciaceae) with polyspored asci. Herzogia 10: 29-37.

Giralt, M. \& Mayrhofer, H. 1995. Some corticolous and lignicolous species of the genus Rinodina (lichenized Ascomy- 
cetes, Physciaceae) lacking secondary lichen compounds and vegetative propagules in Southern Europe and adjacent regions. Biblioth. Lichenol. 57: 127-160.

GonZÁLeZ-Bueno, A. 1988. Los estudios criptogámicos en España (1800-1820): una aproximación a la escuela botánica de A. J. Cavanilles. Llull 11: 51-74.

GonZÁlez-Bueno, A. \& Rico, V. J. 1991. La introducción de las teorías de E. Acharius (1757-1819) en la liquenología española. Acta Bot. Malacitana 16(1): 141-148.

GonZÁLEZ-Fragoso, R. 1883. Apuntes para la flora de la provincia de Sevilla. Anal. Soc. Esp. Hist. Nat. 12: 293-420.

GrubE, M. \& Giralt, M. 1996. Studies on some species of $A r$ thothelium occurring in the western Mediterranean. Lichenologist 28(1): 15-36.

Gueidan, C.; RouX, C. \& LutZoni, F. 2007. Using a multigene phylogenetic analysis to assess generic delineation and character evolution in Verrucariaceae (Verrucariales, Ascomycota). Mycological Research 111: 1145-1168.

Guerra, J.; Ros, R. M.; Cano, M. J. \& Casares, M. 1995. Gypsiferous outcrops in SE Spain, refuges of rare, vulnerable and endangered bryophytes and lichens. Cryptogam. Bryol. Lichénol. 16(2): 125-135.

GutiérREZ, L. \& CASARES, M. 1994. Flora liquénica de los yesos miocénicos de la provincia de Almería (España). Candollea 49(2): 343-358.

Hafellner, J. \& SANCHO, L. G. 1990. Über einige lichenicole Pilze und Flechten aus den Gebirgen Zentralspaniens und den Ostalpen. Herzogia 8: 363-382.

Hansen, E. S. 1993. Collema substellatum and Fulgensia desertorum, new to Greenland. Lichenologist 25(4): 451454.

Hernández Gallego, Y.; Díaz Espejo, A. \& Rowe, J. G. 1998. Distribución de los macrolíquenes corticícolas y su relación con la vegetación en el Parque Natural de los Alcornocales (Cádiz, S de España). Acta Bot. Malacitana 23: 43-50.

Hernández Gallego, Y. \& Muñoz Reinoso, J. C. 2005. Líquenes psammófilos de Las Naves de la Reserva Biológica de Doñana (Huelva). Mediterranea. Serie de Estudios Mediterraneos. Época II, $n^{0}$ 18:1-31.

Herrera, M.; Aragón, G., Prieto, M. \& Belinchón, R. 2007. Diversidad liquénica de las islas Chafarinas, España Bot. Complut: 31: 5-11.

HonegGer, R.; Zippler, U.; SCherRer, S. \& Dyer, P. S. 2004. Genetic diversity in Xanthoria parietina (L.) Th. Fr. (lichen-forming ascomycete) from worldwide locations. $\mathrm{Li}$ chenologist 38: 381-390.

IBÁÑEZ, I. \& Burgaz, A. R. 1998. Epiphytic species of the Lecanora subfusca group (Lecanoraceae) in Spain. Nova Hedwigia 67(1-2): 45-58.

KaLB, K 1970. Fulgensia klementii spec. nov. - eine neue Art der Gattung Fulgensia. Herzogia 1: 439-440.

Kraichak, E.; Parnmen, S.; Lücking, R. \& Lumbsch, H. T. 2014. Gintarasia and Xalocoa, two new genera to accommodate temperate to subtropical species in the predominantly tropical Graphidaceae (Ostropales, Ascomycota). Austr. Sys. Bot. 26(6): 466-474.
KunZE, G. 1846. Chloris Austro-Hispanica e collectionibus willkommianis, a m. Majo 1544 and finem m. Majo 1545 factis. Flora (Regensb.) 29: 86-89.

KuKWA, M. 2011. The lichen genus Ochrolechia in Europe. University of Gdafsk, Polonia.

Lallave, P. 1820. Descripción de algunos líquenes nuevos. Periodico Soc. Med.-Quirur. Cádiz 1: 99-117.

LÁZARO-IBIZA, B. 1898a, b. Nota sobre algunos líquenes de España y Portugal. Actas Soc. Esp. Hist Nat.: 180-186; 200-205.

LLimona, X. 1974. Las comunidades de líquenes de los yesos de España. Universidad de Barcelona, Secretariado de Publicationes, Barcelona.

LLimona, X. 1975. Xanthoria resendei Poelt et Tav. en el SE de España; fitosociología y corología. An. Inst. Bot. Cavanilles 32: 909-922.

LLIMONA, X. \& EGEA, J. M. 1985. Las comunidades liquénicas de las superficies de escorrentía de las rocas silíceas mediterráneas. Anales Jard. Bot. Madrid. 41(2): 429-444.

Llimona, X. \& Hladun, N. 2001. Checklist of the lichens and lichenicolous fungi of the Iberian Peninsula and Balearic Islands. Bocconea 14: 5-581.

LLImona, X. \& Werner, R. G. 1975. Quelques lichens nouveaux ou intéressants de la Sierra de Gata (Almeria, SE de l'Espagne). Acta Phytotax. Barcinonensia 16: 1-32.

Llop, E. \& Gómez-BoleA, A. 1999. Bacidia parathalassica (Bacidiaceae, Lecanorales), a new Mediterranean corticolous lichen. Mycotaxon 72: 79-89.

LLop, E. 2006. Waynea cretica, a new species from the Mediterranean Region. Lichenologist 38(6): 519-527.

LLop, E. 2007. Lecanorales. Bacidaceae I. Bacidia y Bacidina. Flora Liquenológica Ibérica, 2. Sociedad Española de Liquenología.

Llop, E. \& HLADUn, N. 2002. A key to Iberian species of the genus Bacidia with notes on some species. Biblioth. Lichenol. 82: 91-98.

LóPEZ DE SILANES, M. E. 2004. Ramonia. Flora Liquenológica Ibérica, 3. Sociedad Española de Liquenología.

LópeZ de Silanes, M. E. \& AlVAReZ, J. 2004. Gyalecta Flora Liquenológica Ibérica, 3. Sociedad Española de Liquenología.

López de Silanes, M. E.; Carballal, R. \& Paz-Bermúdez, G. 2010. Parmeliella. Flora Liquenológica Ibérica, 6. Sociedad Española de Liquenología.

López de Silanes, M. E.; Etayo, J. \& Paz-Bermúdez, G. 2009. Pronectria pilosa (Hyperocreaceae) sp. nov. and other lichenicolous fungi found on Collemataceae in the Iberian Peninsula. Bryologist 109(2): 101-108.

LumBSCH, H. T. 1993. Studien über die Flechtengattung Diploschistes I. Nova Hedwigia 56(1-2): 227-236.

LumBscH, H. T. et al. 2011. One hundred new species of lichenized fungi: a signature of undiscovered global diversity. Phytotaxa 18: 1-127.

Lumbsch, H. T.; Schmitz, K. E. \& Feige, G. B. 1991. «Lecanora» opiniconensis: a placodioid member of the Parmeliaceae (lichenized Ascomycotina). Mycotaxon 42: 77-87.

Martín, M. P.; WinKA, K.; LLIMONA, X. \& LumbSCH, H. T. 2000. Evaluation of morphological variation in the lichen Diplos- 
chistes ocellatus (Ascomycota, Ostropales): Evidence from nuclear rDNA ITS sequence data. Plant Biology 2: 571-578.

MarTínez, I I 1999. Taxonomía del género Peltigera Willd. (Ascomycetes liquenizados) en la Península Ibérica y estudio de sus hongos liquenícolas. Ruizia 15:1-200.

MartíneZ, I. \& Aragón, G. 1994. Cartografía corológica de líquenes ibéricos, I. Bot. Complut. 19: 139-156.

MARTíNEZ, I. \& ARAGón, G. 2004. The Lecanora varia group in Spain: species with amphithecial cortex. Bryologist 107(2): 222-230.

MARTíNeZ, I. \& HAFELLnER, J. 1998. Lichens and lichenolocous fungi on Peltigera in the Iberian Peninsula and the Canary Islands. Mycotaxon 69: 271-310.

Martín-OSorio, M. E. 1988. Líquenes epífitos de pinsapos. Cádiz Iberoamérica. Dip. Cádiz 6: 23-32.

Martínez-Sánchez, J. J.; Casares-Porcel, M.; Guerra, J.; GuTIÉrREZ-CARRETERo, L.; Ros, R. M.; HERNÁNDEZ-BASTIDA, J. \& CANo, M. J. 1994. A special habitat for bryophytes and lichens in the arid zones of Spain. Lindbergia 19(2-3): 116-121

Meyer, B. 2002. Die flechtengattung Clauzadea. Sendtnera 8: 85-154.

Molina, M. C.; Crespo, A.; Blanco, O.; Lumbsch, H. T. \& HawksworTh, D. L. 2004. Phylogenetic relationchips and species concepts in Parmelia s. str. (Parmeliaceae) inferred from nuclear ITS rDNA and, -tubulin sequences. Lichenologist 36: 37-54.

MoReno, P. P. \& EGEA, J. M. 1989. Fragmenta chorologica occidentalia, lichenes, 2512-2527. Anales Jard. Bot. Madrid 47(2): 467-469.

MoReno, P. P. \& EGEA, J. M. 1992. Estudios sobre el complejo Anema-Thyrea-Peccania en el sureste de la Península Ibérica y Norte de África. Acta Bot. Barcinonensia 41: 1-66.

MotTeRSHEAD, D. \& LuCAS, G. 2000. The role of lichens in inhibiting erosion of a soluble rock. Lichenologist 32: 601609.

MuÑIz, D. \& HLadun, N. 2007. Mycocalicium llimonae, a new species from the Iberian Peninsula. Lichenologist 39(3): 205-210.

MuÑIz, D. \& HLadun, N. 2011. Calicioides. Flora Liquenológica Ibérica, 7. Sociedad Española de Liquenología.

Navarro-Rosinés, P. \& Hladun, N. 1990. El género Sarcopyrenia Nyl. (Ascomycetes liquenícolas) en Europa y norte de África. Candollea 45: 469-489.

Navarro-Rosinés, P.; RouX, C. \& Casares, M. 1995. Hongos liquenícolas de Squamarina II: sobre la identidad de «Didymella» crozalsiana (Ascomicetes). Criptogamie, Bryol. Lichénol. 16(2): 99-103.

Navarro-RosinÉs, P.; RouX, C. \& Giralt, M. 1998. Validación de Wernerella maheui (R.-G. Werner) Nav.-Ros., Cl. Roux $\&$ Giralt (Dothideales, Ascomycetes) y adición de una nueva localidad. Bull. Soc. Linn. Prov. 49: 137-138.

NAVÁs, L. 1901. Notas liquenológicas II. El género Parmelia en España. Bol. Real Soc. Esp. Hist. Nat. 1: 310-317.

NAVÁs, L. 1904. Notas liquenológicas IV. Los Cladoniáceos de España. Bol. Real Soc. Esp. Hist. Nat. 4: 226-236.

Nordin, A. 2000. Taxonomy and phylogeny of Buellia species with pluriseptate spores (Lecanorales, Ascomycotina). Acta Univ. Ups. Symb. Bot. Ups. 33: 1-117.

OtÁlora, M. A. G; Aragón, G.; Martínez, I. \& Molina, M. C. 2004. A new corticolous species of Leptogium (Collemataceae) from Spain. Lichenologist 36: 197-202.

Otálora, M. A. G; Aragón, G.; Martínez, I. \& Molina, M. C. \& Lutzoni, F. 2008. Phylogenetic relationships and taxonomy of the Leptogium lichenoides group (Collemataceae, Ascomycota) in Europe. Taxon 57(3): 907-921.

Paz-Bermúdez, G. 2004. A new species of Parmotrema (Ascomycota: Parmeliaceae) from Portugal. Mycotaxon 89: 505-508.

Paz-Bermúdez, G.; Carballal, R. \& Valcárcel, C. P. 2006. Caloplaca peregrina (Teloschistaceae), a synomym of $C$. pellodella. Bryologist 109(2): 185-187.

Paz-Bermúdez, G.; Carballal, R. \& López de Silanes, M. E. 2010a. Fuscopannaria. Flora Liquenológica Ibérica, 6. Sociedad Española de Liquenología.

Paz-Bermúdez, G.; Carballal, R. \& López de Silanes, M. E. 2010b. Vahliella. Flora Liquenológica Ibérica, 6. Sociedad Española de Liquenología.

Pedreño, J. G.; Moreno, P. P. \& EgeA, J. M. 1987. Revisión de los géneros Hyperphyscia, Phaeophyscia, Physcia y Physconia en el Sur de España. Acta Bot. Malacitana 12: 45-57.

Peinado Lorca, M. \& Rivas-Martínez, S. (Eds.) 1987. La vegetación de España. Colección Aula Abierta. Universidad de Alcalá de Henares, Madrid.

Pereira, I.; Casares, M. \& Llimona, X. 1987. Aportación al conocimiento de los líquenes hidrófilos de la Sierra Nevada (Granada, S. de España). Cryptog. Bryol. Lichénol. 8(3): 263-273.

Pérez Valcárcel, C.; Carballal, R. \& Paz-Bermúdez, G. 2010. Coccocarpia. Flora Liquenológica Ibérica, 6. Sociedad Española de Liquenología.

Pino-Bodas, R.; Martín, M. P. \& Burgaz, A. R. 2010. Insight into the Cladonia convoluta-C. foliacea (Cladoniaceae, Ascomycota) complex and related species, revealed through morphological, biochemical and phylogetic analysis. Sist. Biodiv. 8(4): 575-586.

Pino-Bodas, R.; Burgaz, A. R.; Martín, M. P. \& Lumbsch, H. T. 2012a. Species delimitations in the Cladonia cariosa group (Cladoniaceae, Ascomycota). Lichenologist 44(1): 121-135.

Pino-Bodas, R., Martín, M. P., Burgaz A. R. 2012b. Cladonia subturgida and C. iberica (Cladoniaceae) form a single species morphological and chemically polymorphic. Mycological Progress 11: 269-278.

Pintado, A.; Sancho, L. G.; Green, T. G. A.; Blanquer, J. M. \& LÁZARO, R. 2005. Functional ecology of the biological soil crust in semiarid SE Spain: sun and shade populations of Diploschistes diacapsis (Ach.) Lumbsch. Lichenologist 37: 425-432.

Poelt, J. 1965. Uber einige Artengruppen der Flechtengattungen Caloplaca und Fulgensia. Mitteil. Bot. Staatssammlung München 5: 571-607. 
Poelt, J. \& KRÜGER, U. 1970. Die Verbreitungsverhaltnisse der Flechtengattung Squamarina in Europa. Feddes Repert. 81: 187-201.

Prieto, M.; Aragón, G. \& Martínez, I. 2010. The genus Catapyrenium s. lat. (Verrucariaceae) in the Iberian Peninsula and the Balearic Islands. Lichenologist 42(6): 637-684.

Prieto, M.; Martínez, I. \& Aragón, G. 2010. The genus Placidiopsis in the Iberian Peninsula and the Balearic Islands. Mycotaxon 114: 463-472.

Prieto, P. \& Espinosa, P. 1971. Ascolíquenes de los Peñones de San Francisco. Sierra Nevada. Cuad. Cien. Biol. Univ. Granada 1: 95-98.

Rico, V. J.; Aragón, G. \& Esnault, J. 2007. Aspicilia uxoris, an epiphytic species from Algeria, Morocco and Spain. $\mathrm{Li}$ chenologist 39(2): 109-119.

Rico, V. J. \& Barrasa, J. M. 2011. Basidiomycota liquenizados y liquenícolas. Agaricales y Cantharellales. Flora Liquenológica Ibérica, 9. Sociedad Española de Liquenología.

Rico, V. J.; Calatayud, V. \& Giralt, M. 2003. Buellia tesserata and Dimelaena radiata, two closely related species. $\mathrm{Li}$ chenologist 35(2): 117-124.

Rivas-MartínEZ, S. 1987. Memoria del mapa de series de vegetación de España. ICONA, Madrid.

Rivas-Martínez, S.; DÍAz, T. E.; FERnÁNDEZ-GonzÁlez, F.; IZCO, J.; LoIdi, J.; Lousã, M. \& PenAs, A. 2002. Vascular plant communities of Spain and Portugal. Itinera Geobot. 15(1): $5-432$.

Rowe, J. G.; Casares, M. \& EgeA, J. M. 1996. Excursión de la SEL a los Parques Naturales de la Sierra de Grazalema y los Alcornocales. (16-20 de septiembre de 1996). Granada.

Rowe, J. G. \& EGEA, J. M. 1985a. Contribución al estudio liquenológico de Andalucía occidental II: Estudio de las comunidades sobre granitoides de la zona más térmica de la provincia de Sevilla. Anales Biol. Fac. Biol. Univ. Murcia 6: $33-40$

Rowe, J. G. \& EgeA, J. M. 1985b. Contribución al estudio liquenológico del Sur de España, I: Líquenes silicícolas de la zona más térmica de la provincia de Sevilla. Lazaroa 8: 333 352.

Rowe, J. G. \& EGEA, J. M. 1986. Líquenes silicícolas de las sierras costeras occidentales de la comarca de Algeciras (Cádiz, España). Acta Bot. Malacitana 11: 55-74.

Rowe, J. G. \& EGEA, J. M. 1987a. Líquenes silicícolas de Sierra Morena I. Acta Bot. Malacitana 12: 59-66.

Rowe, J. G. \& EgeA, J. M. 1987b. Líquenes silicícolas de la Sierra del Aljibe (provincia de Cádiz, Sur de España). Stvdia Botanica 6: 89-94.

Rowe, J. G. \& EgEA, J. M. 1988. Líquenes del Parque Nacional de Grazalema. I. Silicícolas. Acta Bot. Malacitana 13:279289.

Rowe, J. G. \& SAinz-Jiménez, C. 1988. Colonisation of mosaics by lichens: the case study of Italica (Spain). Stud. Geobot. 8: 65-71.

Rowe, J. G.; Silvestre, S. \& Llimona, X. 1982. Estudio liquenológico del barranco de Linares. Collect. Bot. (Barcelona) 13(1): 313-317.
SÁiz-Jiménez, C.; Grimalt, J.; García-Rowe, J. \& Ortega-CALV0, J. J. 1991. Analytical pyrolysis of lichen thalli. Symbiosis 11(2-3): 313-326.

SAmpaIO, G. (1917) 1970d. Os liquenes espanhoes do herbarium Willkomm. Anais Fac. Sci. Porto 50: 67-79. Congreso de Sevilla, Assoc. Españ. para el progreso de los Ciencias 1917: 135-144.

Sarrión, F.; Aragón, G. \& Burgaz, A. R. 1999. Studies on mazaediate lichens and calicioid fungi of the Iberian Peninsula. Mycotaxon 71: 169-198.

Sarrión, F. \& Burgaz, A. R. 1995. Comunidades lignícolas del sector central de Sierra Morena (SW de España). Cryptogam. Bryol. Lichénol. 16(2): 137-144.

Sarrión, F. J., Burgaz, A. R. \& Tibell, L. 2007. Calicium chlorosporum new to Europe. Bot. Complut.: 31: 23-25.

SEaward, M. R. D. 1983. Lichens of Malaga Province, S. Spain. Nova Hedwigia 37: 325-345.

SEAWARD, M. R. D. \& ARvidson, L. 1997. Additions to the lichen flora of Malaga, S. Spain. Nova Hedwigia 64(1-2): 129-135.

Sequeiros, M. V.; Rowe, J. G. \& Silvestre, S. 1986. Aportación al conocimiento de los líquenes epífitos de la provincia de Cádiz, I. Stvdia Bot. 5: 85-104.

Silvestre, S. \& García-Rowe, J. 1982. Líquenes en los herbarios Boutelou, de la Universidad de Sevilla y del antiguo Museo de Historia Natural de Sevilla. Collect. Bot. 13(1): 375-380.

Sohrabi, M.; Stenroos, S.; Myllys, L.; Søchting, U.; Ahti, T. \& HYvÖNEN, J. 2013. Phylogeny and taxonomy of the 'manna lichens'. Mycol. Progress 12: 231-269.

SOUZA-Egipsy, V.; Wierzchos, J.; García-Ramos, J. V \& AsCASO C. 2002. Chemical and ultrastructural features of the lichen-volcanic/sedimentary rock interface in a semiarid region (Almería, Spain). Lichenologist 34: 155-167.

TimDaL, E. 1988. Glyphopeltis eburina and Xanthopsorella llimonae are Glyphopeltis ligustica, comb. nov. Mycotaxon 31(1): 101-102.

TimdaL, E. 1991. A monograph of the genus Toninia (Lecideaceae, Ascomycetes). Opera Botanica 110: 1-137.

Torrente, P. \& EGEA, J. M. 1989a. La familia Opegraphaceae en el área mediterránea de la Península Ibérica y Norte de África. Biblioth. Lichenol. 32: 1-282.

Torrente, P. \& EgeA, J. M. 1989b. Opegrapha celtidicola (Jatta) Jatta nombre correcto para Opegrapha betulinoides B. de Lesd. y O. thallincola B. de Lesd. Cryptogam. Bryol. Lichénol. 10(4): 313-317.

Tretiach, M. \& Hafellner, J. 1998. A new species of Catillaria from coastal Mediterranean regions. Lichenologist 30(3): 221-229.

Tretiach, M. \& Muggia, L. 2006. Caloplaca badioreagens, endolithic lichen from Italy. Lichenologist 38(3): 223-229.

Trinkaus, U. \& Mayrhofer, H. 2000. Revision der Buellia epigaea-Gruppe (lichenisierte Ascomycete, Physciaceae). I. Die Arten der Nordhemisphäre. Nova Hedwigia 71: 271314.

Valcárcel, C. P. \& López de Silanes, M. E. 2010. Especies acuáticas del género Verrucaria s.l. (Ascomycetes liqueni- 
zados) en la península Ibérica. Nova Act. Ci. Compostelana, Biol. 19: 5-20.

VAN DEN Boom, P. P. G. \& Rico, V. J. 2006. Caloplaca squamuloisidiata, a new lichen species from Portugal and Spain. Lichenologist 38(6): 529-535

Werner, R. G. 1975. Etude écologique et phytogéographique sur les lichens de l'Espagne méridionale. Rév. Bryol. $\mathrm{Li}$ chénol. 41(1): 55-82.
Werner, R. G. 1979. La flore lichénique de la cordillère bético-rifaine. Collect. Bot. (Barcelona) 11: 401-463.

Winka, K.; Ahlberg, C.; Eriksson, O. E. 1998. Are there lichenized Ostropales - Lichenologist 30(4-5): 455-462.

Zamora, J. C.; Pérez-Ortega, S. \& Rico, V. J. 2011. Tremella macrobasidiata (Basidiomycota, Tremellales), a new lichenicolous fungus from the Iberian Peninsula. Lichenologist 43(5): 407-415. 